\title{
A Comprehensive Study on LPWANs With a Focus on the Potential of LoRa/LoRaWAN Systems
}

This paper was downloaded from TechRxiv (https://www.techrxiv.org).

\section{LICENSE}

CC BY-SA 4.0

SUBMISSION DATE / POSTED DATE

$22-10-2021 / 26-10-2021$

\section{CITATION}

Milarokostas, Christos; Tsolkas, Dimitris; Passas, Nikos; Merakos, Lazaros (2021): A Comprehensive Study on LPWANs With a Focus on the Potential of LoRa/LoRaWAN Systems. TechRxiv. Preprint. https://doi.org/10.36227/techrxiv.16853893.v1

$\mathrm{DOI}$

10.36227/techrxiv.16853893.v1 


\title{
A Comprehensive Study on LPWANs With a Focus on the Potential of LoRa/LoRaWAN Systems
}

\author{
Christos Milarokostas, Dimitris Tsolkas, Nikos Passas, and Lazaros Merakos
}

\begin{abstract}
The Internet of Things (IoT) ecosystem is emerging rapidly. From the connectivity perspective, it is the vehicle towards connecting massive number of devices to the Internet; whereas, from the application perspective, it paves the way towards collecting huge amounts of data and enabling novel information-based services. The connectivity infrastructure that will support the IoT ecosystem is composed of both expansions applied in the well-deployed mobile network infrastructures, and dedicated deployments provided by vertical-specific setups. In the latter case, the Low-Power Wide Area Networks (LPWANs) have become a cost-effective solution, incorporating efficient technologies for wireless connectivity and control. In this context, a comprehensive study is provided to assist on the currently increasing research and development interest in the area of LPWANs. The study provides state-of-the-art work as well as research directions and tools for the most representative LPWAN technologies. Supporting the concept of openness in the IoT ecosystem, the study targets connectivity solutions that operate in unlicensed spectrum bands, such as the LoRa/LoRaWAN systems, and describes the most recent cloud and open-sourced solutions for IoT service and data management.
\end{abstract}

Index Terms-IoT, WSAN, LPWAN, LoRa, LoRaWAN

\section{INTRODUCTION}

O NE of the key targets of beyond 2020 networks is the interconnection of numerous devices to support innovative applications with heterogeneous requirements. This target has been described in the International Mobile Telecommunications 2020 (IMT-2020) ${ }^{1}$ report [1] as one of the three most demanding scenarios for the $5 \mathrm{G}$ era; under the term: massive Machine Type Communications (mMTC). In this context, Internet of Things (IoT) technology [2] serves as key enabler for the realization of the mMTC scenario.

Primarily, IoT has been defined as a global infrastructure for the information society, enabling advanced services, by interconnecting (physical and virtual) things based on existing and evolving interoperable information and communication technologies [3]. Based on this definition, various terms have been coined to highlight i) the application perspective, as for instance, the terms Broadband IoT and Critical IoT, and ii) the network perspective, as for instance, the terms Massive IoT [4] and Network of Things (NoT) [5], [6]. In all the cases, the message is one: in the coming years, the massiveness of IoT devices is expected to be a major challenge. Recent estimations foresee that by the end of 2030 about 500 billion IoT devices will be deployed [7].

C. Milarokostas, D. Tsolkas, N. Passas and L. Merakos are with the Department of Informatics and Telecommunications, National and Kapodistrian University of Athens (NKUA), Athens 15784 Greece (e-mail: chmil@di.uoa.gr; dtsolkas@di.uoa.gr; passas@di.uoa.gr; merakos@di.uoa.gr).

Manuscript to be submitted for review.

${ }^{1}$ The full list of acronyms used in this document is provided in an annex.
In this context, two main approaches are developed. On the one hand, mobile networks (having high coverage from multiple Base Stations (BSs) already in the field) and local area networks (covering well-enough indoor deployments) expand their capabilities, towards including in the pallet of their services IoT communications. 5G New Radio for Reduced Capability (NR RedCap) devices is the most recent advancement of that expansion [8]. On the other hand, the domain of developing IoT dedicated networks, having as main representative the Low-Power Wide Area Networks (LPWANs) [9], is evolving notably.

LPWANs target mainly the deployment of Gateways (GWs), operating as access points, to support End Devices (EDs) that mainly act as sensors (for gathering data of interest) and/or as actuators (for performing an action). As such, LPWANs can be considered as a type of Wireless Sensor and Actuator Networks (WSANs) [10]. From the application perspective, LPWANs support applications with low rate requirements, which can be provided by EDs with low energy demands that remain operational in the field for a long term (a few years), with no charging or battery replacement [11]. Since limited functionality is requested for the EDs and long range (wide area) is covered (hence, a small amount of GWs is required), the LPWANs have become a cost-effective solution for IoT.

The scope of this paper is to present LPWANs as major candidates for the support of the IoT ecosystem, by decomposing various related technologies and revealing research challenges and directions. To this end, extra focus is applied on the Long Range (LoRa)/LoRa Wide Area Network (LoRaWAN) systems, which appears to be the most prominent solution for unlicensed wireless connectivity in LPWANs. With initial velocity from existing surveys in the area, ([12], [13], [14], [15], [16], [17], [18], [19], [20], [21], [22]), we strive for an up-to-date comprehensive review on LPWANs, by providing valuable insights on the underlay technologies and state-ofthe-art directions for research and development. The adopted approach is reflected in the diagram of Fig.1; with the solidcolored path the adopted presentation approach is indicated, where each box represents a core section of the survey, whereas the auxiliary tabular boxes represent self-contained overviews (provided in separated sections) to support the main path.

The remaining of this paper is organized as follows. In Section II, fundamental characteristics of LPWANs are described. Those characteristics are positioned against characteristics of other networking families. In Section III, the key technologies that support LPWANs are presented. In Section IV, a comprehensive comparison of those technologies is provided, in terms of achievable performance and supported capabilities. 


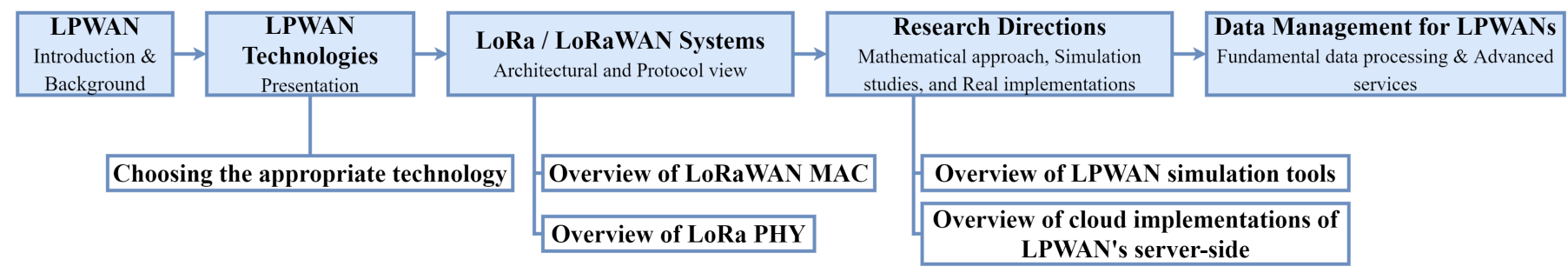

Fig. 1. Block diagram of the structure of this survey. Solid-colored boxes represent the content in the core sections, whereas the tabular boxes reflect self-contained supporting overviews provided in separated sections.

Section $\mathrm{V}$ focuses on LoRa/LoRaWAN systems, delivering an overview of the related protocol stack. Section VI and Section VII delve more into those systems, providing an overview of LoRaWAN and LoRa, respectively. In Section VIII, key considerations in LoRa/LoRaWAN research and development studies are presented. Section IX and Section $\mathrm{X}$ include directions for conducting simulations and utilising cloud services for LPWAN, respectively. Section XI, deals with data management for LPWANs. Conclusions are drawn in Section XII.

\section{BACKGROUND ON LPWANS}

LPWANs define a wireless networking family, supported from various technologies, separated from the other wellestablished wireless networks technologies developed for mobile communications and short range communications. As such they have been considered as part of the $3^{\text {rd }}$ generation of IoT networking, focusing on communication of things with cloud services [23]. Fig.2 compares the key wireless technology families in terms of range, data rate and power efficiency. As it can be observed in Fig.2, LPWAN technologies are the winners in terms of range and energy consumption.

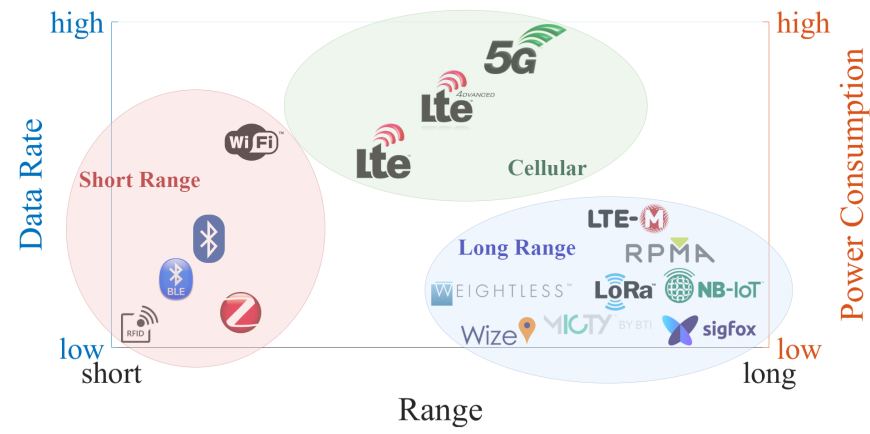

Fig. 2. Wireless technology families with respect to range, data rate and power efficiency

Well established LPWAN technologies are: the Extended Coverage-Global System for Mobile communications-IoT (EC-GSM-IoT), the Long Term Evolution-Machine type communication (LTE-M), the Narrowband-IoT (NB-IoT), the MY IoT (MIOTY), the Random Phase Multiple Access (RPMA), the Wize, the Weightless, the Sigfox and the Long Range (LoRa). The first three technologies (i.e., EC-GSM-IoT, LTEM, NB-IoT) have been defined by 3rd Generation Partnership Project (3GPP), whereas the rest ones are non-3GPP standardized. This splitting criterion also splits the technologies to those that use the licensed part of the spectrum (3GPP technologies) and those that operate in licensed-free spectrum (non-3GPP technologies).

From the architectural perspective, LPWANs are mainly composed of three types of nodes, namely the EDs and the GWs that compose the access-side of the system, as well as the Network/Application Servers, that compose the server-side of the system. EDs are deployed in massive numbers and tend to broadcast their messages in the hope of being received by a GW that covers the area. GWs forward these messages to the server-side of the system, where numerous functions at network and application level take place. At the server side, a security server can also be present along with the application server. Although, nomenclature might be different from one technology to another, an LPWAN system architecture can be usually described by a structure similar to the one depicted in Fig.3.

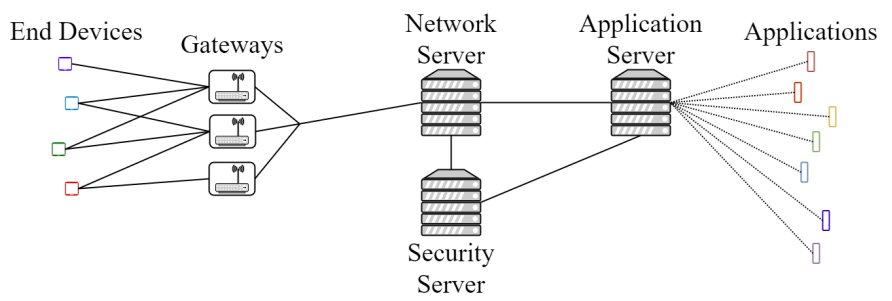

Fig. 3. A generic LPWAN system architecture

From the functional perspective, all LPWANs technologies need to meet certain demands in order to be acknowledged as such. Energy efficiency, long range communication, low-cost operation and up-link dominance are the main demands (or operating characteristics) of all LPWAN technologies.

\section{A. Energy Efficiency}

For all LPWAN technologies the main energy draining factor is the transmission process, hence an appropriate radio interface and a careful transmission planning is essential for the longevity of the system. Furthermore, lightweight protocols are needed to achieve not only minimization of the number of transmissions, but also minimization of each transmission length. For specific scenarios, EDs are powered by solar panels, moving towards an energy harvesting approach, offboarding an important factor of the network management functionality. 


\section{B. Long Range}

As expected by the networking paradigm's name, long range support is a prerequisite for an LPWAN technology. Practically, LPWANs refer to systems that establish links in the order of $10^{3}$ and $10^{4}$ meters. Furthermore, experimental setups have seen communication in the order of $10^{5}$ meters. Such distances are achieved thanks to robust Physical layer protocols and tolerant behavior in the receiving side of the link.

\section{Low cost}

Focusing on the access part of the network, on the one hand, the GWs are deployed in low numbers (due to the long range) and operate as packet forwarders, pushing the main functionality back to the server-side. This minimizes the cost of the GWs. On the other hand of the access network, EDs are deployed in massive numbers and, thus, the minimization of their development cost is performed by constraining the functionality and limiting the hardware/software needs. Indeed, EDs can be built from scratch using the appropriate radio module and a common development board like Arduino, Raspberry Pi and BeagleBoard.

In the server/control-side of the network, for the LPWANs various server components (e.g., application server, security server) are expected, having, however, low impact on the overall cost since the development approach is based in-principle in open source technologies and integrates code snippets and stacks that are available in public software repositories.

\section{UL dominance}

LPWAN access part borrows the principles of the Wireless Sensor Networks (WSNs) [24] and as such it is characterized as UL dominant; that is, the most transmissions are performed from EDs to GWs. Typically, EDs are deployed on the field and are expected to provide the server-side of the system with information obtained by their sensors, based on a periodical or event-driven scheme. It should be noted that contrary to the typical mesh or ad-hoc network topology that is used in WSNs, LPWANs follow a star system development [25].

\section{LPWAN TECHNOLOGIES}

\section{A. EC-GSM-IoT}

EC-GSM-IoT technology was first introduced by 3GPP in Release 13, and further improvements have been added in recent Releases. The main target of the technology was to use existing mobile network infrastructure and functionality (mainly 2G/GSM) for establishing long range IoT communications.

Key point in developing the technology was to maintain advanced features of GSM and General Packet Radio Service (GPRS), while extending the coverage area of these systems and optimizing the protocol for IoT devices [26]. More specifically, EC-GSM-IoT is based on Enhanced GPRS (EGPRS), a wireless cellular technology of '2.75G' that serves as an improvement of GSM in terms of data rate. GSM as a system offers extended coverage compared to other cellular technologies. Its improvement, eGPRS, maintains this advantage and increases system's data rate. EC-GSM-IoT utilizes the two cellular technologies pros, by exploiting the coverage benefit with the sufficient data rates of EGPRS or Enhanced Data rates for Global Evolution (EDGE). Data rate of EDGE is placed at $384 \mathrm{~kb} / \mathrm{s}$, which is beyond EC-GSM-IoT's maximum data rate of $240 \mathrm{~kb} / \mathrm{s}$.

Contrary to GSM and EDGE, EC-GSM-IoT uses enhanced Discontinuous Reception (eDRX) of 52min compared to GSM's DRX of $11 \mathrm{~min}$, performs less operations during inactive periods and utilizes a Power Saving Mode (PSM), leading to extended ED lifetime [27]. Other features of ECGSM-IoT are the optimization of system's information that is used and the nonexistence of redundant re-transmissions; factors that are considered critical in the effort to reduce device complexity and further improve the battery usage [27]. Also, EC-GSM-IoT is equipped with additional channels to achieve greater coverage and overlaid Code Division Multiple Access (CDMA) scheme for increased capacity [28]. Security improvements on the areas of integrity, authentication of both ends and the ciphering algorithms that are used, classify ECGSM-IoT as LTE-level secure [28].

In the deployment field, EC-GSM-IoT is supported by lowcost EDs that can operate for up to 10 years with an average of $5 \mathrm{~W} / \mathrm{h}$ battery usage. These EDs transmit in multiple GSM frequency bands according to deployment area, from $395 \mathrm{MHz}$ to $1960 \mathrm{MHz}$, using $200 \mathrm{kHz}$ Bandwidth (BW) channels. The deployment of EC-GSM-IoT's channels is performed in a GSM in-band scheme. Message transmissions are performed in two power classes; one being at $23 \mathrm{dBm}$ and the other at $33 \mathrm{dBm}$. The two classes define the two coverage areas cases, with the former having a Maximum Coupling Loss (MCL) of $154 \mathrm{~dB}$ and the latter of $164 \mathrm{~dB}$. Data rate depends on modulation type. Gaussian Minimum Shift Keying (GMSK) offers data rates between $0.35 \mathrm{~kb} / \mathrm{s}$ and $70 \mathrm{~kb} / \mathrm{s}$, while 8 -Phase Shift Keying (8PSK) can go up to $240 \mathrm{~kb} / \mathrm{s}$. These data rates correspond to UL as well as DL. Time Division Multiple Access (TDMA)/Frequency Division Multiple Access (FDMA) are used as multiple access schemes in both DL and UL.

\section{B. LTE-M}

Another Cellular IoT (CIoT) technology that can satisfy the needs of low power and extended coverage is LTE-M. We note that, in the context of this paper, with the term LTE-M, we refer to the common characteristics of LTE-MTC, LTE Cat M1 and LTE-M technologies. LTE-M as CIoT technology utilizes the LTE spectrum for establishing wireless communication links allowing coexistence with other cellular technologies such as $2 \mathrm{G}, 3 \mathrm{G}$ and especially advantages of the $4 \mathrm{G}$ standard.

LTE-M deployment cost and time-to-market is reduced, since LTE's BSs can be used for LTE-M links with a software upgrade. Security and privacy benefits from LTE also exist in LTE-M and issues like confidentiality of end user's identity (practically ED), authentication, integrity of messages and identification of EDs are tackled successfully. Moreover, with improvements added in 3GPP Releases 14 and 15, LTE-M EDs 
with mobility capabilities can be supported, since in-band LTE operation permits handovers from one cell to the other. Other improvements added in 3GPP's Releases 14, 15 and 16 include the standardization of a new device category; namely Cat. 2, that offers substantial enhancements in ED's performance. Furthermore, Voice Over LTE (VoLTE) features were added in Release 14 [29]. Release 15 built upon these improvements and presented new use cases, supporting higher ED mobility, adding a new power class of $14 \mathrm{dBm}$, reducing latency and ED's power consumption, increasing spectral efficiency and improving access control [26]. The evolutionary approach of the technology was continued in Release 16. Worth mentioning enhancements include new power saving techniques for EDs, extended support of EDs' mobility and improvements that targeted coexistence with New Radio (NR), i.e., the $5 \mathrm{G}$ radio interface [30].

Overall, LTE-M is based on a simplified version of LTE protocols and functionality towards reducing ED complexity and cost, and improving power consumption. More precisely, in LTE-M, there is no control channel for wideband operation, Transmission Modes (TM) support is reduced and Hybrid Automatic Repeat Request (HARQ) is also reduced [28], since message size is already limited and the addition of large number of redundant bits for error detection purposes should be restricted.

In the deployment field, LTE-M EDs are able to operate in low power modes and utilize power saving techniques like PSM, eDRX and Connected mode DRX (C-DRX) to live up to 10 years with a $5 \mathrm{~W} / \mathrm{h}$ battery consumption. The frequency bands that are used for message transmissions are cellular, ranging from $462.5 \mathrm{MHz}$ to $2655 \mathrm{MHz}$ and their selection is based on each area's spectrum regulation and operators' decision. The BW is $1.08 \mathrm{MHz}$ and the in-band LTE operation is applied. LTE-M defines two power classes: the class of $23 \mathrm{dBm}$ and the class of $20 \mathrm{dBm}$. Both classes result in the same coverage area, with an MCL of $155.7 \mathrm{~dB}$. Despite the extended area that the system can cover, data rates are the greatest among other LPWAN solution, reaching $1 \mathrm{Mb} / \mathrm{s}$ for both streams. Especially for Cat. 2 devices, data rates of $7 \mathrm{Mb} / \mathrm{s}$ in $\mathrm{UL}$ and $4 \mathrm{Mb} / \mathrm{s}$ in DL using $5 \mathrm{MHz} \mathrm{BW}$, can be reached. For both UL and DL, Quadrature Phase Shift Keying (QPSK) and 16-Quadrature Amplitude Modulation (16-QAM) are utilized for modulation purposes. In UL, Single Carrier-FDMA (SCFDMA) is used as multiple access scheme, whereas in DL the use of Orthogonal FDMA (OFDMA) is preferred; same as in LTE networks. It is worth mentioning, that in both UL and DL techniques like $15 \mathrm{kHz}$ tone spacing and Turbo codes are utilized to increase system's performance [28].

\section{NB-IoT}

NB-IoT is one of the most prominent LPWAN CIoT technologies. NB-IoT defines a radio interface for IoT communications, moving one step further from the EC-GSM-IoT and LTE-M technologies which are solely based on the GSM and LTE radio interfaces, respectively.

NB-IoT is specified in 3GPP Release 13, but much like ECGSM-IoT and LTE-M, future improvements of the technology are included in Releases 14, 15 and 16. More precisely, in Release 14 , a new device power class was established at $14 \mathrm{dBm}$, mobility improvements were introduced and enhancements in power consumption and latency were proposed among other upgrades of the technology [29]. Data rate was also improved to reach the scale of $250 \mathrm{~kb} / \mathrm{s}$ for both UL (for multi-tone deployment) and DL. Release 15 brought further improvements, with system's range increase to $120 \mathrm{~km}$, support of microcells, pico-cells and femto-cells, wake-up enhancements for EDs that operate in DRX or eDRX and other upgrades [26]. Release 16 expanded the technology even further, with key improvements among others being power saving techniques in wake-up procedure of EDs, directions towards Self Organizing Networks (SONs), greater mobility support and coexistence of the technology with NR [30].

As a 3GPP CIoT technology, NB-IoT utilizes bands in the licensed part of the spectrum, both in the sub- $\mathrm{GHz}$ region, like B8 or B26 and above $1 \mathrm{GHz}$, like B66 or B74. The BW of the system is $180 \mathrm{kHz}$. Three modes of operation are defined as depicted in Fig.4 [31]. In the stand alone mode, NB-IoT utilizes part of the spectrum that is used for GSM EDGE Radio Access Network (GERAN) by operating in a GSM carrier. In the guard-band mode, unused resource blocks inside an LTE's carrier are utilized. These blocks are at the edges of the carrier and are typically used for tackling interference issues. Finally, in in-band operation, NB-IoT uses an LTE Physical Resource Block (PRB) for its needs; hence the size of $180 \mathrm{kHz} \mathrm{BW}$, since LTE uses 12 subcarriers of $15 \mathrm{kHz}$ width each. The mode of operation is agnostic to ED when it connects to the network [32].
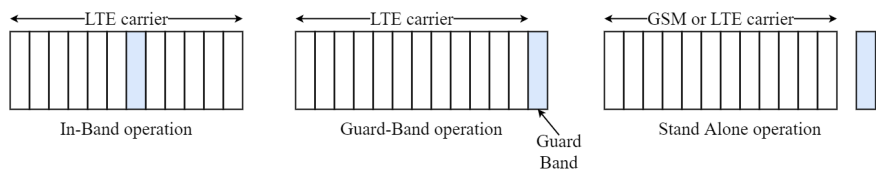

Fig. 4. NB-IoT modes of operation

In the deployment field, NB-IoT uses the same power saving techniques as in LTE-M; namely PSM, eDRX and C-DRX. Battery life is calculated to approximately 10 years, with solutions being designed to last even more with the typical $5 \mathrm{~W} / \mathrm{h}$ battery usage. Key advantage of NB-IoT is its MCL value, that can go up to $164 \mathrm{~dB}$ for standalone deployment, allowing extremely extended coverage. Supported modulation schemes include QPSK in both UL (multi-tone) and DL, and $\frac{\pi}{4}$-QPSK or $\frac{\pi}{2}$-Binary PSK (BPSK) in UL (single-tone) [20]. Being able to coexist and utilize LTE bands, NB-IoT uses SC-FDMA in UL and OFDMA in DL for multiple access, however the area of access mechanisms in NB-IoT is undergoing extensive research [33]. Again, to improve system's performance, techniques like $15 \mathrm{kHz}$ tone spacing in both streams (or even $3.75 \mathrm{kHz}$ tone spacing in single-tone UL operation) and Turbo coding in UL are used.

\section{MIOTY}

Moving to non-3GPP technologies that operate in unlicensed bands, the first examined candidate is MIOTY. MIOTY 
is an LPWAN standard that follows one of the 3 European Telecommunications Standards Institute (ETSI) specifications for the so called Low Throughput Networks (LTN). In [34], ETSI defines 3 LTN protocol families; namely Lfour family, Telegram Splitting Ultra Narrow Band (TS-UNB) family and Dynamic Downlink Ultra Narrow Band (DD-UNB) family. MIOTY is a realization of the $2^{\text {nd }}$ protocol family, TS-UNB, mainly implemented by BTI.

MIOTY can offer communications services to EDs with high mobility, of up to $120 \mathrm{~km} / \mathrm{h}$. The underlying LTN protocol, i.e., the TS-UNB, offers great advantages in the area of robustness against co-channel interference; a factor highly critical for links operating in the unlicensed part of the spectrum. This robustness is achieved thanks to GW's ability to decode the entire message using a portion of the received packets. Adding to Telegram Splitting (TS) benefits, the split messages require less time to transmit, resulting in reduced Time On Air (ToA), and, hence, an improvement in battery usage. As a TSUNB technology, MIOTY uses Telegram Splitting Multiple Access (TSMA) for allowing more than one EDs to access the communication channel. TS as a concept is explained in [34], [35] and [36]. In TS, the packets to be transmitted are first divided into several smaller sub-packets and then they are sent in a non-continuous fashion, with each transmission being separated by the other in time domain. The sub-packets are distributed pseudorandomly in both time and frequency within a certain radio frame.

In the deployment field, two power classes are supported by MIOTY, based on the frequency band that is used for the communication; either $868 \mathrm{MHz}$ in Europe or $915 \mathrm{MHz}$ in North America. That leads to the $14 \mathrm{dBm}$ power class for the European band and the $23.3 \mathrm{dBm}$ class for the North American band. With these power classes and proper configuration, EDs can operate in the field for about 20 years. The link budget of a MIOTY link is calculated at $154 \mathrm{~dB}$, with a receiver sensitivity of $139 \mathrm{dBm}$ [34]. Data rate by official statement is $0.514 \mathrm{~kb} / \mathrm{s}$, but estimations set it at $0.4 \mathrm{~kb} / \mathrm{s}$ using a $\mathrm{BW}$ of $200 \mathrm{kHz}$.

\section{E. RPMA}

RPMA was invented and patented [37] by Theodore J. Myers with On-Ramp assignee; the company that is now called Ingenu. RPMA, is explained as a concept in [16] and [38]. It is actually a Direct Sequence Spread Spectrum (DSSS) technique that pseudorandomly delays the transmissions of EDs to minimize overlapping occurrences, resulting in increased Signal to Interference and Noise Ratio (SINR) value for every node.

Contrary to other popular LPWAN technologies and especially the unlicensed ones, RPMA does not make use of the sub-GHz frequency bands for link establishment. RPMA utilizes the globally available $2.4 \mathrm{GHz}$ band for wireless transmission. Despite not having the added advantage of high penetration due to low frequency that other LPWAN solutions have, RPMA behaves extremely well in terms of coverage. For tackling the issue of interference in a band that operates also one of the most widely used wireless technologies, i.e., WiFi (IEEE 802.11), RPMA utilizes its high robustness and a frequency plan that assigns small channels in-between the channels of WiFi [39]. To provide multiple access in the wireless channel, RPMA uses the namesake multiple access scheme.

Another key feature of RPMA is its solid security and privacy support. EDs communicate with RPMA's BSs having ensured authentication for both end points of the link, messages are sent encrypted by the EDs and the backhaul communication of the system is achieved via secure Secure Sockets Layer (SSL)/Transport Layer Security (TLS) connections.

In the deployment field, the BW that is used by RPMA is significantly higher than the typical case of LPWANs, reaching the size of $1 \mathrm{MHz}$ [40]. Regarding transmission power, EDs transmit at a maximum of $21 \mathrm{dBm}$ in both US and Europe, while RPMA BSs can transmit at $30 \mathrm{dBm}$ in US and at $21 \mathrm{dBm}$ in Europe [41]. In this power class and by optimizing the usage of EDs for longevity, nodes can last on the field for more than 10 years, while estimates state that can last for about 20 years with rare message transmissions and small-sized packets. A key advantage is its extremely high link budget as well. It can reach $168 \mathrm{~dB}$, with a receiver sensitivity of $142 \mathrm{dBm}$ for BSs and $133 \mathrm{dBm}$ for EDs. However, the data rate is estimated to be $8 \mathrm{~kb} / \mathrm{s}$ at maximum [21].

Interestingly, as of 2018, Ingenu has decided to offer RPMA as a Platform-as-a-Service (PaaS) solution [42].

\section{F. Weightless}

Weightless Special Interest Group (SIG) started developing 3 solutions for LPWA networks; namely Weightless -W, -N, -P. Weightless-W operates in the TV White Space (TVWS) region, making it a worth examining solution, but the different regulations in different parts of the world regarding TVWS spectrum complicate its deployment. Weightless-N operates in the sub-GHz unlicensed region and seems as a promising solution due to its numerous similarities with Sigfox; one of the most popular LPWANs. The technology is used by NWave, however Weighltess-N's link budget is considered to be floating [43], leading Weightless SIG to another alternative. The suggested solution is Weightless-P [44], a technology that this paper focuses on.

Weightless-P as a technology supports fully acknowledged (ACK) bi-directional links and various techniques can be used among others to offer Quality of Service (QoS), like Forward Error Correction (FEC) in block interleaving mode, Cyclic Redundancy Code (CRC) and ARQ. It, also, offers various advanced security and privacy features, with mutual authentication via Advanced Encryption Standard (AES) 128/256, message integrity, confidentiality via AES 128/256 and secure Over The Air (OTA) key exchange being supported [45].

In the deployment field, the BSs that implement Weightless$P$ standard transmit at a typical power of $27 \mathrm{dBm}$, while being able to transmit also at $30 \mathrm{dBm}$. EDs have a lower transmit power, at $14 \mathrm{dBm}$, being also capable for $30 \mathrm{dBm}$ transmissions. What is more important, is the power consumption of EDs at idle state, which is placed at $100 \mu \mathrm{W}$. With this power usage, an ED can last for 3-8 years [46]. Even though the technology can theoretically operate at both licensed and unlicensed spectrum, 
for the time being, it operates at unlicensed bands in the sub$\mathrm{GHz}$ region. These bands are: $138 \mathrm{MHz}, 433 \mathrm{MHz}, 470 \mathrm{MHz}$, $780 \mathrm{MHz}, 868 \mathrm{MHz}, 915 \mathrm{MHz}$ and $923 \mathrm{MHz}$. The choice of band is subjected to each area's frequency regulation. BW is set at $12.5 \mathrm{kHz}$ or $100 \mathrm{kHz}$ for UL, while at DL broadcast, unicast and multicast channels exist, using $100 \mathrm{kHz}, 50 \mathrm{kHz}$ or optionally $12.5 \mathrm{kHz}$.

Link budget of Weightless-P can reach at most $153 \mathrm{~dB}$, assuming an Additive White Gaussian Noise (AWGN) channel. Its value depends on transmission parameters, with the highest figure being obtained for UL transmissions when the receiver sensitivity is set at $-135.5 \mathrm{dBm}$, with a required Signal to Noise Ratio (SNR) of $-17.5 \mathrm{~dB}$, for a typical Bit Error Rate (BER) of $10^{4}$, while Coding Rate (CR) is set at $\frac{1}{2}$ and Spreading Factor (SF) at 8. The $153 \mathrm{~dB}$ value is true, if Offset QPSK (OQPSK) is used as a modulation method. The other modulation method that the technology utilizes is GMSK with a Bandwidthsymbol Time (BT) product of 0.3 . These modulations are used in both UL and DL. For multiple access, UL messages use TDMA and FDMA combined, whereas in DL only FDMA is utilized. Considering the data rates, the maximum value is $100 \mathrm{~kb} / \mathrm{s}$ in both streams for $100 \mathrm{kHz}$ BW, GMSK modulation and proper CR selection, but falls to $10 \mathrm{~kb} / \mathrm{s}$ at $\max$ in $\mathrm{UL}$ for $12.5 \mathrm{kHz}$ BW. OQPSK performs worse in terms of data rates; a penalty that has to be paid for the great system range it offers.

\section{G. Wize}

Different from the techniques presented so far, Wize provides, in addition to the Physical layer functionality, a full protocol stack. It also adopts the conventional LPWAN architecture; however the server-side is a data collection system called Head-End, and between the GWs and the EDs, LAN modems could be used to serve multiple EDs. The Wize protocol stack includes four layers; namely the PHY, the Data Link, the Presentation and the Application (APP) layer. Wize specification is supported by Wize Alliance and can be found in [47]. Based on this specification we describe below (following a bottom-up approach) the main aspect of each layer.

In the deployment field, the related PHY layer parameters are described in [48]. Practically, the $169 \mathrm{MHz}$ Industrial, Scientific, Medical (ISM) band is utilized and targets deployments in European region. Six channels are defined, each $12.5 \mathrm{kHz}$ wide, permitting transmissions with a power of up to $27 \mathrm{dBm}$ with the necessity of respecting a duty cycle of $10 \%$. The modulation that is used is either Gaussian Frequency Shift Keying (GFSK) or 4GFSK, both with a BT product of 0.5 . The former modulation can give data rates of either up to $2.4 \mathrm{~kb} / \mathrm{s}$ or $4.8 \mathrm{~kb} / \mathrm{s}$, while the latter can go as high as $6.4 \mathrm{~kb} / \mathrm{s}$. Optimal sensitivity by popular market products is achieved with the $2.4 \mathrm{~kb} / \mathrm{s}$ PHY layer, giving a value of $-119 \mathrm{dBm}$ [49], resulting in a maximum link budget of $146 \mathrm{~dB}$.

On top of PHY layer resides the Wize Data Link layer. Two different packet formats are defined; the DL broadcasting format and the default UL/DL format. In the UL (i.e., the dominant communication direction) multiple transmissions of the same message (repetitions) are allowed to increase the probability of message reception. Possible duplicates that may appear due to time redundancy or broadcasting nature of UL messages are resolved in Head-End. In the DL, unicasting transmissions for device-specific commands or broadcasting software update commands are sent to EDs. As in the most related systems, the DL messages are UL-initiated. Practically, this behavior can be translated into great power management device-wise, since EDs remain idle during non-UL periods (with the exception of waiting for a software update broadcasting), allowing a DL message in a small communication window only shortly after the UL broadcast.

Security aspects and metadata management are performed at the Wize Presentation layer. Regarding system's security, Wize supports both access authentication using AES Cipher-based Message Authentication Code (CMAC) for ED - GW communication and end-to-end authentication using AES CMAC. Encryption using AES Counter (CTR) mode is also provided.

The data plane of the APP layer, is related to the use case supported. Wize targets mainly smart city applications, with gas metering and water management verticals being the most adopted ones. Regardless the use case, application administration options (control plane) are offered as well.

\section{H. Sigfox}

Sigfox is one of the widely adopted non-3GPP technologies for LPWA networks, that owes its name to the namesake company, Sigfox, that first introduced the technology. A comprehensive review of the technology can be found in [50]. Based on the Sigfox set of specifications, EDs can be developed, while the server-side system is also provided by Sigfox and Sigfox Cloud. EDs communicate with BSs via Sigfox links and the backhaul communication is performed within a Virtual Private Network (VPN), via fixed cable links (Digital Subscriber Line (DSL)) as a primary solution and cellular technology as a backup. Satellite support can act as an alternative solution as well [51].

In the deployment field, Sigfox defines three power classes for EDs, according to the region that the technology is used. Currently, there are 7 region zones, named RC1-7. $1^{\text {st }}$ zone consists mostly of European countries and parts of Africa and Middle East, $2^{\text {nd }}$ zone includes North America and parts of South America, $3^{\text {rd }}$ zone is dedicated to Japan, zone 4 covers some other countries of South America and the Asia-Pacific area and zones 5, 6 and 7 are exclusive for South Korea, India and Russia respectively. For RC1, RC3, RC6, RC7 the maximum TP is set at $16 \mathrm{dBm}$, for RC5 is at $14 \mathrm{dBm}$, whereas in $\mathrm{RC} 2, \mathrm{RC} 4$ is at $24 \mathrm{dBm}$. However, TP can differ based on device class. In Sigfox, 4 device classes are defined; namely $\mathrm{U} 0-\mathrm{U} 3$, with $\mathrm{U} 0$ being the strongest and U3 the weakest. For $\mathrm{U} 1$ devices, $\mathrm{TP}$ is in the range of $\left[\mathrm{TP}_{\mathrm{U} 0}-9 \mathrm{~dB}, \mathrm{TP}_{\mathrm{U} 0}-4 \mathrm{~dB}\right]$, for $\mathrm{U} 2$ the range is $\left[\mathrm{TP}_{\mathrm{U} 0}-14 \mathrm{~dB}, \mathrm{TP}_{\mathrm{U} 0}-9 \mathrm{~dB}\right]$ and for $\mathrm{U} 3$ we have a TP below $\mathrm{TP}_{\mathrm{U} 0}-14 \mathrm{~dB}$. This $\mathrm{TP}$, along with other technology parameters, allow battery powered EDs to have an autonomy at the scale of a decade.

Sigfox's frequency plan is also region based and utilizes ISM parts of the spectrum. Devices that operate in $\mathrm{RC} 1$ areas transmit at $868.130 \mathrm{MHz}$, in RC2 at $902.200 \mathrm{MHz}$, in $\mathrm{RC} 3$ at 
923.200MHz, in RC4 at $920.800 \mathrm{MHz}$, in RC5 at $923.300 \mathrm{MHz}$, in RC6 at $865.200 \mathrm{MHz}$ and in RC7 at $868.800 \mathrm{MHz}$. The total BW used in a region is $192 \mathrm{kHz}$ and transmission $\mathrm{BW}$ is only $100 \mathrm{~Hz}$, thus implementing an Ultra Narrow Band (UNB) scheme.

The transmission rate for Sigfox is $600 \mathrm{~b} / \mathrm{s}$ in the DL and either $100 \mathrm{~b} / \mathrm{s}$ or $600 \mathrm{~b} / \mathrm{s}$ in the UL, depending again on the region. RC2 and RC4 support the $600 \mathrm{~b} / \mathrm{s}$ data rate, while the rest of the regions allow UL transmissions of $100 \mathrm{~b} / \mathrm{s}$. These data rates might seem extremely low, but in reality are sufficient enough considering the information that is transmitted and the size of the packets. In Sigfox, packets have a payload size of $0-12 B y t e s$ and a total size of 26Bytes for the UL, and a fixed payload size of 8 Bytes for the DL. As a result, transmission of 26 Bytes with a $100 \mathrm{~b} / \mathrm{s}$ rate needs only $2.08 \mathrm{~s}$. To transmit their messages, devices utilize the available channels in a RandomFDMA (RFDMA) scheme. EDs choose three channels in a pseudo-random fashion to transmit their message 3 times in 3 different frequencies in an effort to increase the chance of their message to be receipt by at least one BS, thus decreasing the amount of lost information and improving the QoS.

The total number of messages that an ED can send in one day depends on several parameters including the region, the payload size and the bit rate. In RC1 and RC7 a duty cycle of $1 \%$ is imposed due to regulations, in $\mathrm{RC} 2$ and $\mathrm{RC} 4$ a Frequency Hopping ( $\mathrm{FH})$ scheme is adopted as described earlier with the 3 times transmission approach and in RC3 and RC5 Listen Before Talk (LBT) is preferred. Shortly examining the European region case, for a 12Bytes payload (26Bytes total size) message transmissions in $100 \mathrm{~b} / \mathrm{s}$ with a $1 \%$ duty cycle, an ED can transmit 6 messages an hour and 140 messages a day. In DL, messages are less often and are initiated by a UL message, with DL duty cycle being at $10 \%$ and the maximum number of messages per day and per device being 4 . The sensitivity of a Sigfox BS is also region depended and for a typical RC1 transmission can reach $-142 \mathrm{dBm}$, resulting in a theoretical link budget of $163.3 \mathrm{~dB}$. However, a typical link budget of $156 \mathrm{~dB}$ can be considered reasonable.

\section{LoRa/LoRaWAN}

LoRa as a technology refers to the Physical (PHY) layer of the protocol stack, and more precisely, to a proprietary Chirp Spread Spectrum (CSS) modulation, that has been developed by Semtech as described in [52]. Despite being tightly connected with LoRaWAN, it should be noted that, while LoRa is the PHY layer technology, LoRaWAN is an open Medium Access Control (MAC) protocol residing on top of it and maintained by LoRa Alliance.

LoRa EDs may transmit at a maximum power of $16 \mathrm{dBm}$, whereas GWs have two power classes; one defined by the same maximum TP with the EDs and the other at a maximum value of $27 \mathrm{dBm}$. Chipsets built by Semtech support transmission of packets at ISM bands, on a region-depended fashion or by utilizing universally available bands, like $2.4 \mathrm{GHz}$. Transmission frequencies are set by the protocol used in MAC layer. LoRaWAN, which is the main proposal in this area, defines 8 ISM regions [53], as described later. Bandwidth is also non fixed and can be either at $125 \mathrm{kHz}, 250 \mathrm{kHz}$ or $500 \mathrm{kHz}$ in several widely used chipset implementations, while others can go down to $7.8 \mathrm{kHz}$ [54].

A number of LoRa parameters, like BW, might differ from one link to another, resulting in a link budget that can be adapted to each use case, with its maximum value reaching $154 \mathrm{~dB}$. Other parameters that can be configured in a LoRa link include CR, SF, Carrier Frequency (CF) and TP [55], which can be set in a value lower than the maximum allowed if the channel quality permits it. CF refers to the band used to carry the information and although typically adjustable, due to the extended usage of LoRaWAN protocol, the CF is dictated by deployment region. Regarding SF, the options are 6; from SF7 to SF12, while CR should be selected from the set $\left\{\frac{4}{5}, \frac{4}{6}, \frac{4}{7}\right.$, $\left.\frac{4}{8}\right\}$. Data rate is variable and can take values in the area of $[0.162 \mathrm{~kb} / \mathrm{s}, 50 \mathrm{~kb} / \mathrm{s}]$, however LoRaWAN-based systems that implement LoRa in PHY layer can achieve data rates of up to $5.47,11$ or $21.9 \mathrm{~kb} / \mathrm{s}$ according to deployment region (despite Semtech chipsets having greater capabilities), whereas the maximum value of $50 \mathrm{~kb} / \mathrm{s}$ is achieved via Frequency Shift Keying (FSK) modulation. LoRaWAN supports also Long Range - Frequency Hopping Spread Spectrum (LR-FHSS) for some regions, with a maximum bit rate of either $0.162 \mathrm{~kb} / \mathrm{s}$ or $0.325 \mathrm{~kb} / \mathrm{s}$, again in a region-based fashion [53].

Since LoRa operates in ISM bands, transmissions should respect the use of medium by other technologies. Common regulations regarding LoRa is the moderation of duty cycle, i.e: percentage of time that an ED is active (where active refers to transmitting), with values of $0.1 \%, 1 \%$ and $10 \%$ being common. Practically, duty cycle regulates the ToA of a LoRa transmitted packet for several regions. The ToA regulator role may also be performed by dwell time, whereas some regions are subjected to both regulations. Instead of duty cycle, LBT is also an option in a few occasions.

Multiple access is subjected to the protocol used above LoRa. Considering the typical case of LoRaWAN protocol, EDs are accessing the transmission medium in an ALOHA scheme with variable frame sizes [56], however its performance has been measured to be greater than ALOHA either due to SF orthogonality, other transmission characteristics (e.g., capture effect) or due to improvements suggested [57], [58]. SF orthogonality in LoRaWAN should be taken into account when discussing about multiple access due to its imperfect nature [59], [60]. Several research efforts examine different approaches to the field of multiple access [61], [62], [63], [64], [65]. DL is also permitted in LoRaWAN, either in unicast form for every ED class specified in protocol (Class $\mathrm{A}, \mathrm{B}, \mathrm{C}$ ) or multicast for some ED classes (Class B, C).

\section{J. Other LPWAN technologies}

The LPWAN area is developing at such a rapid pace, that more technologies are emerging. As discussed, one of Weigthless SIG proposals is Weightless-N, which is implemented by a smart cities oriented system, called Nwave [66]. Major industry players, like Sony and Amazon, offer their solutions; namely Eltres [67] and Sidewalk [68] respectively (Sidewalk operates with LoRa and/or FSK and/or Bluetooth 
Low Energy (BLE)). Sensor Networks Over White spaces (SNOW) [69] is another worth considering option for LPWAN deployment, while Wi-Fi HaLow [18], [70] is gaining more and more attention. DASH7 [71] is a well-established protocol, supported by DASH7 Alliance. The interested reader can also search for alternatives like Telensa, NB-Fi, iQRF, Thingstream, Helium, SAT4M2M, Hiberband and Wi-SUN.

\section{Choosing the ApPropriate TeChnology}

\section{A. Operational characteristics}

Based on the presentation of the main LPWAN technologies, provided in the previous section, TABLEI summarizes their key characteristics. As it can be observed in TABLEI, all the technologies have a link budget of over $146 \mathrm{~dB}$, justifying their capabilities of covering the needs of LPWAN systems, while they share very common operational characteristics with some little variations.

\section{B. Existing deployments}

Besides the very common operational characteristics that are shared among the LPWAN technologies, four candidates are currently showing the greatest acceptance, namely: LTE-M, NB-IoT, Sigfox and LoRa. This trend is backed up by market research conducted by IoT Analytics [72]. According to their estimates, in 2019, more than 9 out of 10 LPWAN systems $(92 \%)$ were deployments based on those four technologies. An analysis of how this percentage of $92 \%$ is distributed among the 4 dominant technologies is provided in Fig.5.

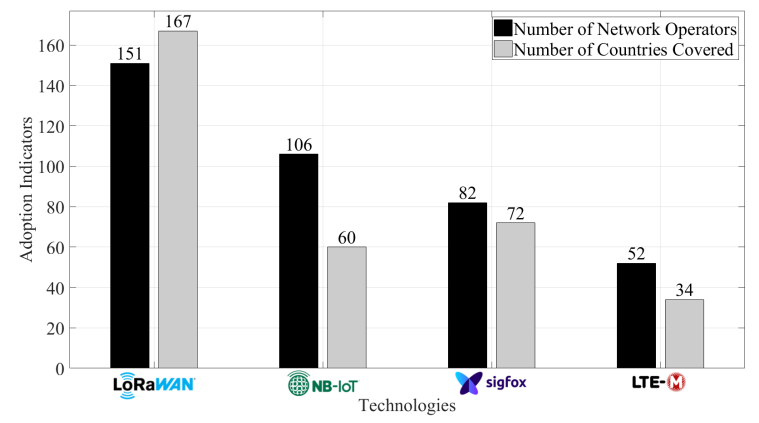

Fig. 5. Number of operators and countries covered by the top 4 LPWAN technologies as of July 2021

As it can be observed in Fig.5, LoRaWAN technology dominates against the other technologies, regarding both the number of LoRaWAN network operators and the number of countries with established LoRaWAN networks. Part of that domination should be attributed to cost reasons, since NB-IoT and LTE-M utilize the licensed spectrum and make use of more computing capable and energy demanding BSs, while Sigfox networks operate on a subscription-based scheme. Another reason could be the publicly available LoRaWAN protocol, which contradicts the typical approach for non-3GPP technologies. In contrary, NB-IoT seems to be a very popular option among the 3GPP technologies, which are deployed by network operators. Focusing on the Sigfox's number of operators, 23 out of the 82 depicted in Fig.5 are a Sigfox carrier, distinguishing itself country-based (e.g., Sigfox France, Sigfox USA, Sigfox Hellas, etc.). That leads to 59 Sigfox operators that are not immediate derivatives of Sigfox.

In Fig.5 an interesting pattern can be observed as well. For the LoRaWAN technology the number of countries covered exceeds the amount of network operators, revealing the higher applicability of licence-free LPWAN technologies in cross-country deployment scenarios. Interestingly, in Fig.5 the depicted numbers of the LoRaWAN technology refer to deployments where LoRaWAN MAC on top of LoRa PHY is used, meaning that there might be even more LoRa systems established, with other protocol stacks on top of them, highlighting the lead of LoRa in the course of LPWAN PHY technologies.

\section{Key performance indicators}

Continuing with the study of the main LPWAN technologies, a comparison between the two main non-3GPP candidates and also between the two main candidates in general, would lead to even more conclusions. To start of, an examination of how LoRa and Sigfox perform in key IoT areas is depicted in Fig.6, as described in [19].

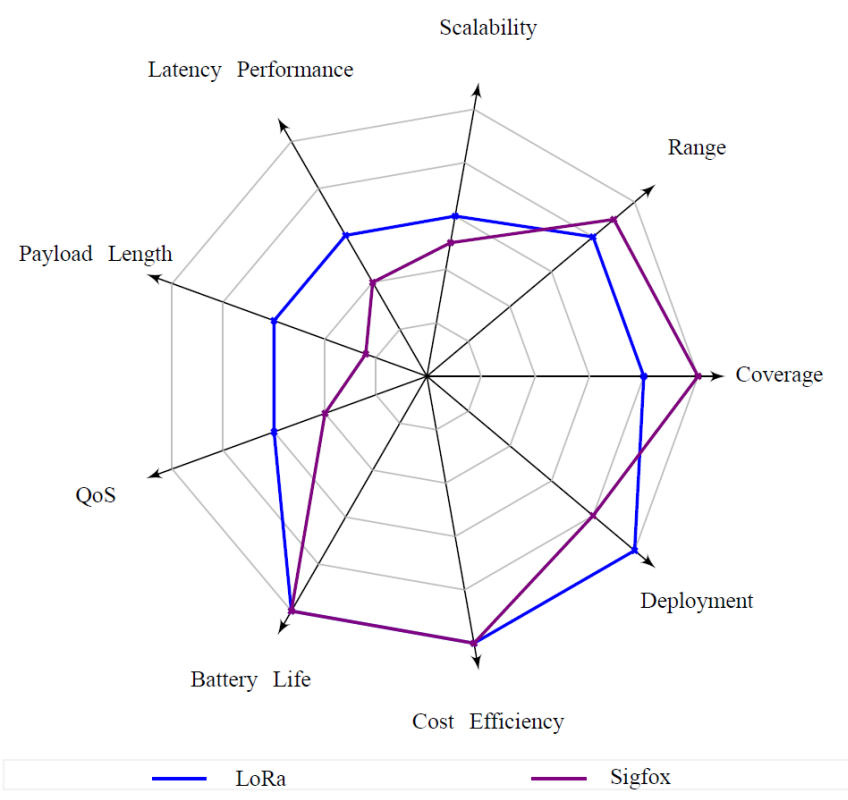

Fig. 6. Comparison of LoRa and Sigfox regarding IoT KPI's [19]

Fig.6 showcases the superiority of Sigfox systems in the areas of range, which is backed up by TABLEI data, where Sigfox has a slightly higher link budget; and coverage, which is justified by the widely deployed Sigfox's BSs. On the other hand, LoRa outperforms or matches Sigfox in the remaining 7 examined KPIs. LoRa is considered optimal in terms of system deployment, whereas it performs more satisfactory in terms of scalability, latency, amount of information carried in each packet and QoS. Both technologies perform the best in the areas of battery life and cost, however research conducted in [19] takes into account issues like spectrum usage, BS or GW cost and ED cost, which are in the same scale for these two technologies. Should we examine other cost factors, then 
TABLE I

COMPARISON OF LPWAN TECHNOLOGIES

\begin{tabular}{|c|c|c|c|c|c|c|c|}
\hline Technology & $\begin{array}{c}\text { Modulation } \\
\text { [UL / DL] }\end{array}$ & $\begin{array}{c}\text { Multiple Access } \\
\text { [UL / DL] }\end{array}$ & $\begin{array}{l}\text { Frequency } \\
\text { (MHz) }\end{array}$ & $\begin{array}{c}\text { Bandwidth } \\
\text { (kHz) } \\
\text { [UL / DL] }\end{array}$ & $\begin{array}{l}\text { Maximum Data } \\
\text { Rate (kb/s) } \\
\text { [UL / DL] }\end{array}$ & $\begin{array}{c}\text { Maximum } \\
\text { Transmission } \\
\text { Power (dBm) } \\
{[\text { UL / DL] }} \\
\end{array}$ & $\begin{array}{l}\text { Maximum } \\
\text { Link Budget } \\
\quad \text { (dB) }\end{array}$ \\
\hline EC-GSM-IoT & $\begin{array}{l}\text { GMSK or } \\
\text { 8PSK }\end{array}$ & $\begin{array}{c}\text { TDMA, FDMA or } \\
\text { CDMA }\end{array}$ & $\begin{array}{c}\text { cellular } \\
\text { bands }\end{array}$ & 200 & $\begin{array}{l}\text { GMSK: } 70 \\
\text { 8PSK: } 240\end{array}$ & 23,33 & $164[\mathrm{MCL}]$ \\
\hline LTE-M & $\begin{array}{l}\text { QPSK or } \\
\text { 16-QAM }\end{array}$ & $\begin{array}{l}\text { SC-FDMA / } \\
\text { OFDMA }\end{array}$ & $\begin{array}{l}\text { cellular } \\
\text { bands }\end{array}$ & $\begin{array}{c}1080 \text { or } \\
5000\end{array}$ & $\begin{array}{c}1000 \text { or } 7000 / \\
1000 \text { or } 4000 \\
\text { (3GPP Release } \\
\text { dependent) }\end{array}$ & $\begin{array}{c}14,20,23 \\
\text { (3GPP Release } \\
\text { dependent) }\end{array}$ & 155.7 [MCL] \\
\hline NB-IoT & $\begin{array}{c}\frac{\pi}{4}-\mathrm{QPSK} \text { or } \\
\frac{\pi}{2}-\mathrm{BPSK} \text { or } \\
\text { QPSK / QPSK }\end{array}$ & $\begin{array}{l}\text { SC-FDMA / } \\
\text { OFDMA }\end{array}$ & $\begin{array}{l}\text { cellular } \\
\text { bands }\end{array}$ & 180 & 250 & $\begin{array}{c}14,23 \\
\text { (3GPP Release } \\
\text { dependent) }\end{array}$ & $164[\mathrm{MCL}]$ \\
\hline MIOTY & TS-UNB & TSMA & $\begin{array}{c}\text { ISM } \\
\text { region } \\
\text { dependent }\end{array}$ & 200 & 0.4 & $\begin{array}{c}14,23.3 \\
\text { (region dependent) }\end{array}$ & 154 \\
\hline RPMA & $\begin{array}{c}\text { DSSS } \\
\text { (RPMA) }\end{array}$ & RPMA & 2400 & 1000 & $0.01-8$ & $\begin{array}{c}21 / 30 \\
21 / 21 \\
\text { (region dependent) }\end{array}$ & 163 \\
\hline Weightless-P & $\begin{array}{l}\text { OQPSK or } \\
\text { GMSK }\end{array}$ & $\begin{array}{c}\text { TDMA and } \\
\text { FDMA / FDMA }\end{array}$ & $\begin{array}{c}\text { ISM } \\
\text { region } \\
\text { dependent }\end{array}$ & $\begin{array}{c}12.5,100 / \\
12.5,50,100\end{array}$ & $\begin{array}{l}10 \text { or } 100 / 100 \\
\text { (BW and modula- } \\
\text { tion dependent) }\end{array}$ & 14 or $30 / 27$ or 30 & 153 \\
\hline Wize & $\begin{array}{l}\text { GFSK or } \\
\text { 4GFSK }\end{array}$ & $\begin{array}{l}\text { ALOHA-like } \\
\text { (unslotted) }\end{array}$ & $\begin{array}{c}\text { ISM } \\
169\end{array}$ & 12.5 & $\begin{array}{c}\text { GFSK: } 2.4,4.8 \\
\text { 4GFSK: } 6.4\end{array}$ & 27 & 146 \\
\hline Sigfox & $\begin{array}{c}\text { DBPSK / } \\
\text { GFSK }\end{array}$ & $\begin{array}{c}\text { ALOHA-like } \\
\text { (unslotted), } \\
\text { Random-FDMA }\end{array}$ & $\begin{array}{c}\text { ISM } \\
\text { region } \\
\text { dependent }\end{array}$ & 0.1 & $\begin{array}{c}0.1 \text { or } 0.6 / 0.6 \\
\text { (region dependent) }\end{array}$ & $\begin{array}{l}14,16,24 \\
\text { (region } \\
\text { dependent) }\end{array}$ & 156 \\
\hline LoRa/LoRaWAN & $\begin{array}{c}\text { LoRa }(\mathrm{CSS}) \text { or } \\
\text { FSK or } \\
\text { LR-FHSS }\end{array}$ & $\begin{array}{l}\text { ALOHA-like } \\
\text { (unslotted) }\end{array}$ & $\begin{array}{c}\text { ISM } \\
\text { region } \\
\text { dependent }\end{array}$ & $\begin{array}{l}125 \\
250 \\
500\end{array}$ & $\begin{array}{l}\text { LoRa: } 5.47,11 \\
\text { or } 21.9 \text { (region } \\
\text { dependent) } \\
\text { FSK: } 50 \\
\text { LR-FHSS: } 0.162 \\
\text { or } 0.325 \text { (region } \\
\text { dependent) }\end{array}$ & $\begin{array}{l}16 / 16 \text { or } 27 \\
\text { (chipset and } \\
\text { region dependent) }\end{array}$ & 154 \\
\hline
\end{tabular}

LoRa might seem as a more attractive solution, since Sigfox is a purely subscription-based system. In reality, both LoRa and Sigfox can be commercialized, but LoRa gives the option for cost-free operation deployments.

Moving to the comparison of LoRa and NB-IoT, we can observe an interesting behavior while looking at Fig.7 [17], [19]. It can be seen that where LoRa lacks performance, NBIoT excels and vice versa. LoRa systems offer great coverage, are easy to deploy, cost efficient and support EDs that can operate for multiple years while being powered by simple batteries. On the other hand, NB-IoT deployments, can reach great distances, are able to scale easier, perform superbly in terms of latency and QoS offered and have a greater payload length. These indicators lead to the conclusion that LoRa and NB-IoT are more of a complementary technologies than competitors.

\section{LORA/LORAWAN SYSTEMS}

The combination of LoRa PHY along with LoRaWAN MAC seems to be a highly efficient and preferable solution for the establishment of LPWAN systems. This is due to the key characteristics of the underlay combined technologies, i.e., the LoRa modulation and the LoRaWAN protocol. Below the LoRa/LoRaWAN systems are further analysed.

\section{A. Architectural view}

From the architectural perspective, a star-of-stars topology is followed in the deployment of the LoRa/LoRaWAN systems,

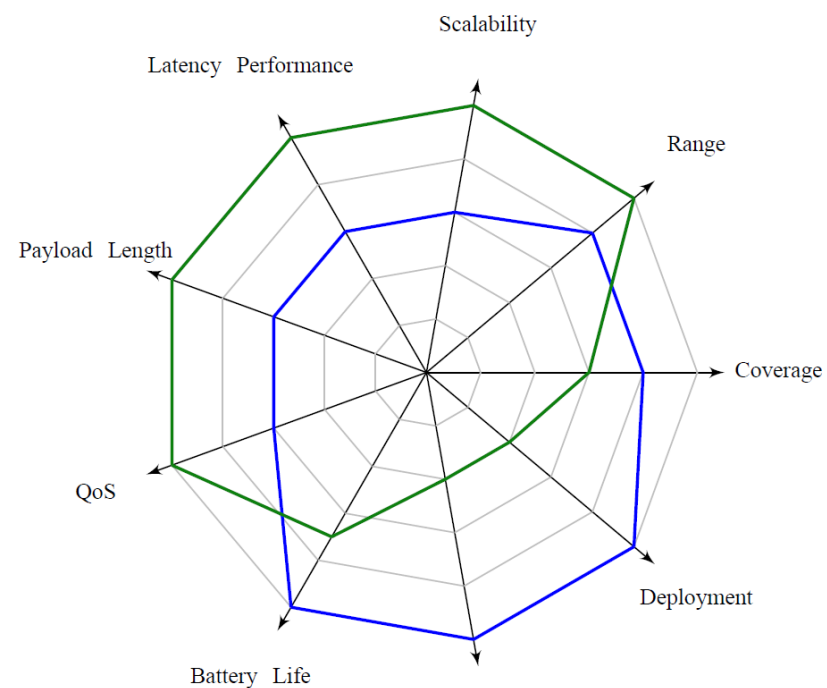

Cost Efficiency

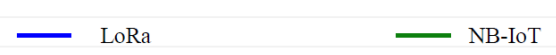

Fig. 7. Comparison of LoRa and NB-IoT regarding IoT KPI's [17], [19]

as it can be observed in Fig.8.

EDs are deployed in a star topology around GWs, and they broadcast their messages by using single-hop loRa links defining the network access side of the architecture. GWs are deployed in a star topology around a central node, called 


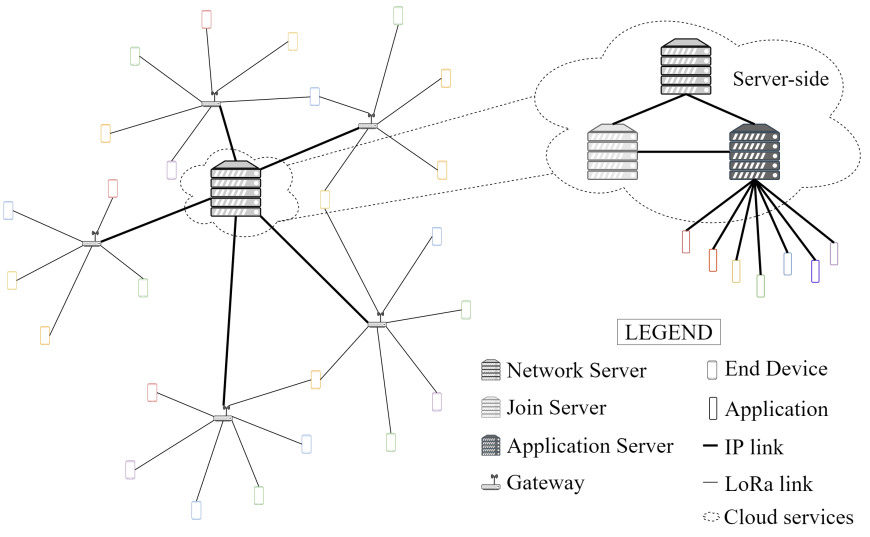

Fig. 8. Architecture and topology of a LoRa/LoRaWAN system

Network Server (NS). The main role of the GWs is to forward UL messages to an NS and distribute DL messages to EDs.

Once the messages arrive to an NS, packet deduplication is applied and then the data are forwarded to an Application Server for application-layer processing of the payload. For the ED to NS connection a separated authentication server can be used, called Join Server. All the entities of the server side of the system (Network, Application, and Join servers) can be hosted either in a single location (singe point of presence) or in a distributed cloud platform.

1) Server side: The NS is the core component of the architecture, containing the intelligence of the deployed network. NS usually resides in a computer system with full computing capabilities, although the advancements in single board computers (like Raspberry Pi) over the recent years, has lead to NS implementations in such systems. The current trend dictates the deployment of NS in a cloud infrastructure along with the rest of architecture's server-side.

NS can be either public or private. A number of industry partners and network operators have allowed access to their public NS, with most-known solution in this area being the NS offered by The Things Network (TTN). However, in many occasions it is preferred to develop a private NS, for managing devices and applications. Solutions by TTN, Chirpstack, Loriot, ResIoT, Actility and others are widely used. These solutions might offer additional components of LoRaWAN system, like an application server, a security or authentication server, a geolocation server and a managing platform.

NS, as the central point of the star-of-stars topology (Fig.8), is responsible for a number of operations, including: node activation, message deduplication, direct communication with applications or with the application server (should it exists), enforcing ED updates via Firmware Update Over The Air (FUOTA) messages, sending MAC commands, and the Adaptive Data Rate (ADR) algorithm. Node activation can be performed either using the Over The Air Activation (OTAA) method or the Activation By Personalization (ABP) method. Both of them are explained later.

Packet deduplication is a critical operation, since arrival of duplicate packets is a commodity. EDs transmit their messages via broadcasting. With GWs' deployment becoming denser, a
UL message can be received by more than one GWs. GWs are designed with no intelligence by default, meaning that they act as plain packet forwarders regarding the upstream traffic. As a result, duplicate packets appear in the NS, adding to its responsibilities the identification and resolution of them. Another possible way of duplicate packets appearance is the loss of ACK packets from NS to ED, i.e., an ED sends UL that requires acknowledgment, NS receives that UL and transmits ACK but ACK never reaches ED, resulting in ED retransmitting the same UL that already exists in NS. Duplicate packets can also emerge due to QoS policy, i.e., an NS may instruct an ED to broadcast the same frame up to 15 times, in an effort to increase its reception probability; a scheme reminiscent to Sigfox's triple UL. A Received Signal Strength Indication (RSSI) deduplication policy is described in [73].

The data received by NS are destined to be used in an application. In this context, NS should be able to provide these data to applications built on top of the network infrastructure. Usually, an application server is deployed for handling such operations, which offers significant advantages i) in terms of NS performance and complexity, since NS has to communicate only with the application server and not with each application separately, and ii) in terms of system scalability, since the addition of a new application requiring a dedicated application server will have no impact on the configuration of NS other than establishing a new communication channel between it and the new application server.

Apart from UL traffic, LoRaWAN supports message transmission from NS to ED. An example of such DL traffic are FUOTA messages. FUOTA [74], a process running on the APP, allows EDs to update their firmware without the need of physical access to the device. Usually, it is supported by a firmware update server and the application server, however the distribution of the update file to EDs is performed by the NS. Another example of DL traffic are the MAC commands initiated by NS for Class A and Class B EDs, as described later. These commands may ask an ED for its state or modify its transmission parameters. For Class B EDs, there are additional beacon-related commands.

Another operation performed by NS is ADR algorithm [75]; an effort to offer the best trade-off of data rate to range for EDs, maximize the capacity of the network and minimize the need for UL ACK messages. When an ED sends UL packets to the GW, the GW forwards them to NS using Internet Protocol (IP) links. The GW does not act on the packet, but it does attach some additional information, like timestamp, RSSI value and SNR level. Based on these metadata for all UL packets from all EDs, NS can have a holistic view of the network and thus decide what is the best SF configuration for each ED.

The selection of SF follows a simple approach: for high link budget, use smaller SFs, thus increasing data rate and reducing link range, message airtime and energy consumption. The opposite is the case with low link budget, where higher values of SF have to be used to achieve longer range communication, with lower data rates and greater energy consumption. The impact of SF configuration in the discussed trade-off will become evident when the performance metrics of a LoRa link 
will be presented. The underlying logic of ADR is based on the position of each ED. An ED that sends messages via a high link budget connection is either positioned in close proximity of GW or has an obstacle-free path, allowing higher data rates and better energy management, without the need of increasing link's range. On the other hand, distant EDs or EDs with established links unfavorable to successful communication with $\mathrm{GW}$, have to sacrifice data rate for a more robust SF configuration.

It should be noted that this ADR scheme with fixed positioned EDs is performed by NS (network-managed); if an ED is in motion, the notion of Blind ADR is utilized [76]. In general, non-stationary EDs decide whether to implement ADR or not, since they have greater knowledge on the current link state compared to the NS. For high mobility EDs, ADR is preferably disabled due to highly variable communication channel. Valuable insights in ADR are given by [77] and [78], whereas an extensive discussion on the topic and the different implementations of ADR can be found in [79].

2) Network access side: EDs and GWs are the components of the access part of the architecture. EDs are usually smallsized, energy-constrained devices with low computing capabilities. These characteristics are imposed by the need to be cost-effective, since they are deployed in massive numbers. EDs can be stationary or perform operations while on move.

Typical building blocks of an ED are: the power unit, the radio module, the memory block, the processor and the sensor(s). EDs can either rely on a fixed power scheme for their operation or be battery-operated. The latter approach is usually preferred, although recent proposals discuss the utilization of small solar panels on top of EDs as a main or supplementary energy solution, towards the realization of energy harvesting WSNs. The radio module has the greatest energy needs relative to other operations like sensing and acting, and as a result the selection of the suitable radio technology and a careful transmission planning are considered essential. An antenna is another component of an ED, but it is common to be considered as a part of its radio interface. In market devices, antenna can be printed.

A memory unit in the order of few MBs or even $\mathrm{KBs}$ is considered adequate for satisfying the needs and the constraints of such a device. The processor of an ED has rather limited capabilities compared to modern processors used in home or enterprise computing systems, but is sufficient enough for an ED and its required operation. Finally, an ED may have multiple sensors, being able to perform several measurements, varying from temperature sensing to position awareness. Sensing is the main operation of EDs, hence the WSN characterization of the access part of IoT systems. The main modules of an ED can be observed in Fig.9. A more sophisticated view of a smart sensor's architecture can be found in [24] and [80]. Recent advancements in microelectronics have lead to the development of chips that can incorporate both radio transceiver and microprocessor for LoRa EDs ${ }^{2}$.

\footnotetext{
${ }^{2}$ https://www.thethingsnetwork.org/article/breaking-down-stm32wl-thecentral-element-of-generic-node-sensor-edition-and-first-dual-core-lorawansystem-on-chip-soc?mc_cid=57d7f74b8f\&mc_eid=8698751288, Accessed: 04 October 2021
}

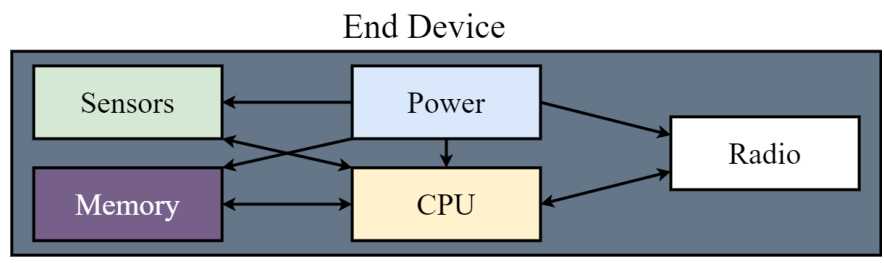

Fig. 9. Main modules of an LPWAN ED

As for GWs, they rely on a fixed power source for their operation. They also have a radio module for LoRa communication, however several other network interfaces, which can be either wireless (e.g., cellular, IEEE 802.11) or wired (e.g., Ethernet) may be a GW component. Of course, an antenna is present, usually being significantly more powerful than EDs' antennas. Computing capabilities are higher than that of EDs. A GW can receive and decode messages by more than one EDs and more than one GWs may receive the messages broadcast by an ED. GWs can receive simultaneously data in different frequencies and different SFs.

\section{B. Protocol stack view}

The LoRa/LoRaWAN protocol stack fits to the main elements of the architecture as described in Fig.10. In Fig.10 the well-known layers of Open Systems Interconnection (OSI) stack, used both for the IP communication between the GW and the network side internal communication, are also depicted.

1) Server side: It is apparent that NS participates in both data and control flows. NS's functionality starts by receiving from GW an APP payload encapsulated in a LoRaWAN frame, which is encapsulated in appropriate types of packets regarding the rest of the OSI protocol stack (L4 to L1). NS performs the necessary decapsulation and according to the type of the message, either it remains in NS (control messages that are not related to authentication actions, and application messages in an architecture that does not include an application server) or it is further forwarded to the server-side: i) to an application server (data flow) or ii) to a join server (authenticationrelated control flow). The reverse order of encapsulation and decapsulation takes place in DL messages.

A key observation is that LoRaWAN resides above transport layer in NS; that is expected since GW to NS and vice versa communication is achieved via links that transfer APP messages encapsulated in transport layer protocol segments (User Datagram Protocol (UDP) is preferred), encapsulated in NET layer protocol datagrams (IP), encapsulated in Data Link layer protocol frames (Ethernet, cellular, IEEE 802.11). A complete definition of system's backend is given in [81].

2) Network access side: The stack in an ED is simpler than that in NS, as expected. In the case of information belonging to data flow, an APP message is encapsulated in a LoRaWAN MAC frame, which is then encapsulated in a LoRa PHY packet to be broadcast via the LoRa radio interface. The broadcast LoRa packets are received by a GW, which performs decapsulation operations to extract the APP payload. The extracted payload is placed in a typical transport layer protocol 


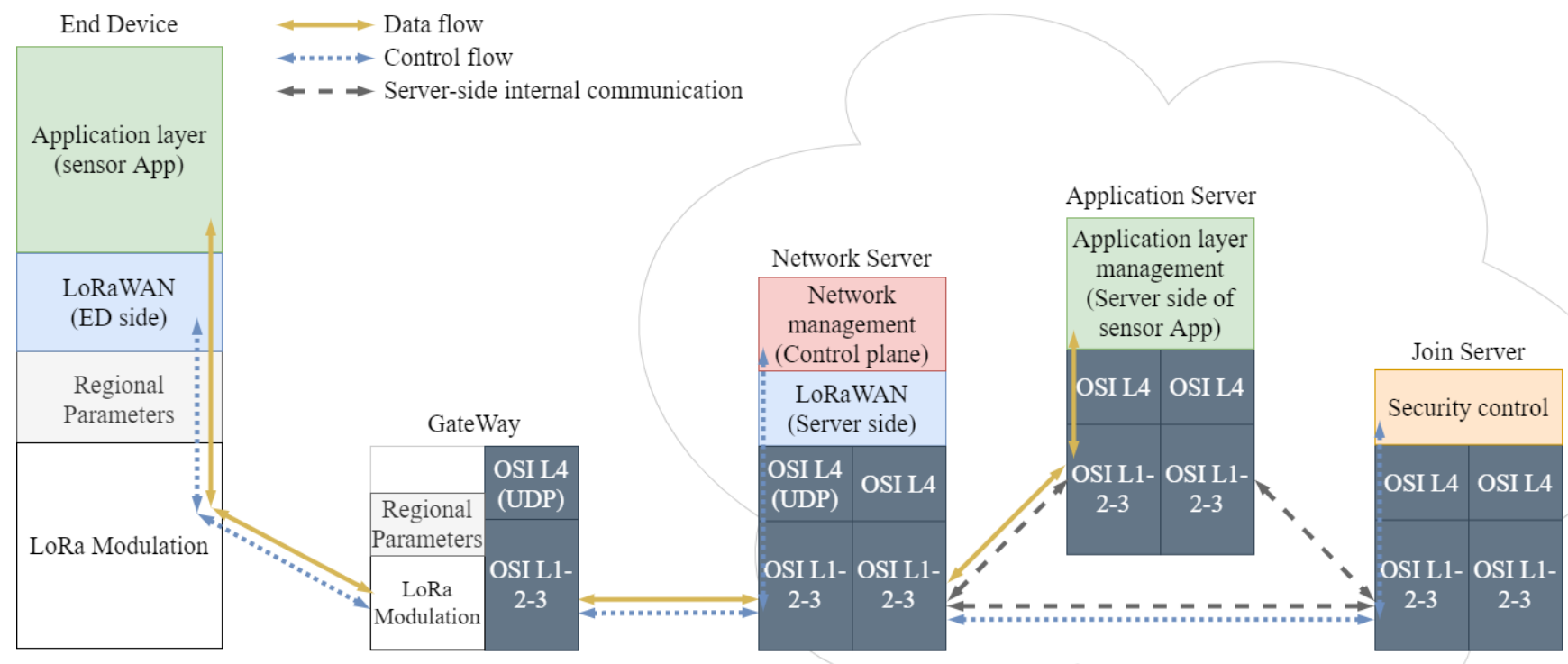

Cloud Infrastructure

Fig. 10. LoRa/LoRaWAN protocol stack mapped in the main elements of the architecture

segment, then it is encapsulated in an IP datagram, and finally, it is encapsulated in the desired Data Link layer protocol frame (Ethernet, cellular, IEEE 802.11) to be transmitted to NS. The reverse operation takes place during DL. For control flow information the APP-related encapsulation and decapsulation may be skipped.

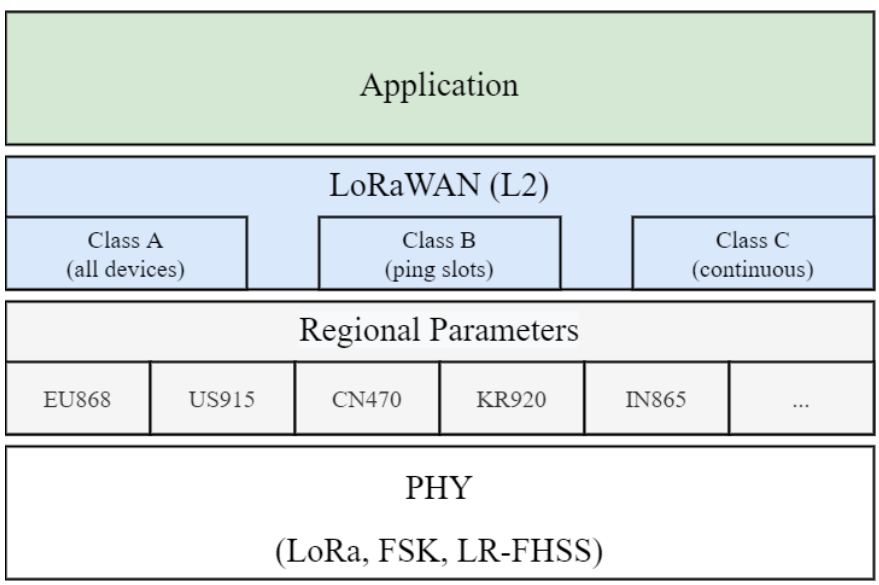

Fig. 11. Protocol stack at a LoRaWAN-enabled End Device [56]

More precisely, at the ED side, the protocol stack can be seen in Fig.11 [56]. A 4-layer stack can be observed, namely the APP, the LoRaWAN, the Regional Parameters and the PHY, which practically can be narrowed down to 3 layers since the Regional Parameters (TABLEII) actually defines which PHY can be supported at which bands. In the PHY layer, LoRa modulation is the main choice. The FSK modulation is supported as well, based on [53]. LR-FHSS [82] is also an option. On top of the PHY, LoRaWAN MAC layer describes 3 device classes, which define the available DL windows and ultimately determine the power consumption scheme of ED.
TABLE II

LoRaWAN Frequency Planning Based on DePloyment Region

\begin{tabular}{|c|c|c|}
\hline Region & Frequency band (MHz) & Abbreviation \\
\hline $\begin{array}{c}\text { Europe \& some } \\
\text { African countries }\end{array}$ & $863-870,433$ & EU868, EU433 \\
\hline North America & $902-928$ & US915 \\
\hline China & $779-787,470-510$ & CN779, CN470 \\
\hline Australia & $915-928$ & AU915 \\
\hline Asia & 923 & AS923 \\
\hline South Korea & $920-923$ & KR920 \\
\hline India & $865-867$ & IN865 \\
\hline Russia & $864-870$ & RU864 \\
\hline
\end{tabular}

The full frequency plan documentation can be found in [53]. In Europe, no plan for the use of the EU433 band exists. On the other hand, EU868 is used extensively for LoRa transmissions. In all regions other than US915 and AU915, 16 communication channels may be used with 3 of them being mandatory to implement, since they can be used for ED joinrequest messages. In TABLEIII, the mandatory channels of EU868 for UL transmission are depicted.

TABLE III

EU868 MANDATORY CHANNELS FOR UL TRANSMISSIONS

\begin{tabular}{|c|}
\hline Channel (MHz) \\
\hline 1. 868.1: LoRa (SF7BW125 up to SF12BW125) \\
\hline 2. 868.3: LoRa (SF7BW125 up to SF12BW125, SF7BW250) \\
\hline 3. 868.5: LoRa (SF7BW125 up to SF12BW125) \\
\hline
\end{tabular}

Regarding DL transmissions, the same channels as UL transmissions may be available, however the default DL channel is set at $869.525 \mathrm{MHz}$ (LoRa, SF9BW125), which permits transmission for GWs operating in higher TP, $27 \mathrm{dBm}(0.5 \mathrm{~W})$.

On top of LoRaWAN MAC layer stands the APP. This is where the applications that are built on top of LoRaWAN infrastructure reside, utilizing the services offered by the layers 
below. A number of use cases can be discussed on this area, and protocols intriguing to be studied do exist. LoRa Alliance has established 6 Working Groups (WGs), that are responsible for the further development of the according vertical sectors. These include all the familiar "smart" applications like agriculture, buildings, cities, industry, logistics and utilities [83].

\section{AN OVERVIEW OF THE LORAWAN MAC PROTOCOL}

LoRaWAN is an open protocol being developed by LoRa Alliance. The protocol is officially defined as:

"LoRaWAN ${ }^{\circledR}$ specification is an LPWA networking protocol designed to wirelessly connect battery operated 'things' to the internet in regional, national or global networks."

LoRa Alliance is an association that has been established since 2015, having Semtech as a founding member and being supported by numerous industry players. More on LoRa Alliance can be found in the corresponding website [84].

\section{A. LoRaWAN device classes}

The main characteristic of the LoRaWAN layer is the establishment of 3 device classes; namely Class A, Class B and Class C. An ED has to transmit in a scheme described by one of these 3 classes in order to be considered as a LoRaWAN device.

Class A stands for All, meaning that all EDs have to be able to implement at least this class to perform LoRaWAN transmissions. Class A defines two open DL windows with a maximum of $15 \mathrm{~s}$ and $16 \mathrm{~s}$ delay respectively after a UL transmission (typical values are $1 \mathrm{~s}$ and $2 \mathrm{~s}$ or in case a joinrequest UL message has been preceded $5 \mathrm{~s}$ and $6 \mathrm{~s}$ ), in which an ED may receive messages from NS for data or control reasons. The $2^{\text {nd }}$ receive window opens in case there was no successful reception during the $1^{\text {st }}$ window. Regarding the UL, Class A EDs can transmit either a data message, a MAC command (initiated by ED or an answer to a previous NS-initiated MAC command) or a combination of them. The transmission scheme can be seen in Fig.12.

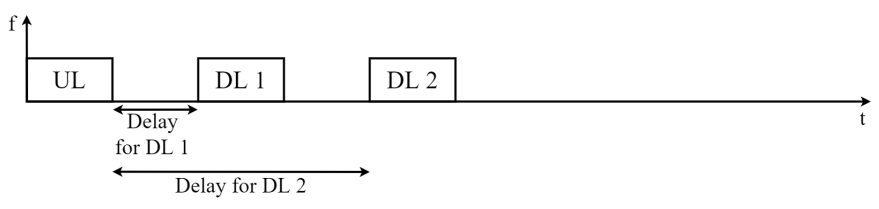

Fig. 12. LoRaWAN Class A

Class B stands for Beacon, meaning that EDs open additional receive windows, based on synchronization beacon frames that are sent either from NS to $\mathrm{ED}$ via $\mathrm{GW}$ or directly from GW to ED. Differentiating from Class A, messages received in Class B's supplementary DL slots can be only datarelated, however additional Class-B specific MAC commands exist that are sent by NS to ED during Class A receive windows. For UL, again there is a set of MAC commands available either as an ED-originated request or as an answer to an NS-originated MAC request. Class A to Class B switching decision originates from ED's APP layer. The transmission scheme can be seen in Fig.13.

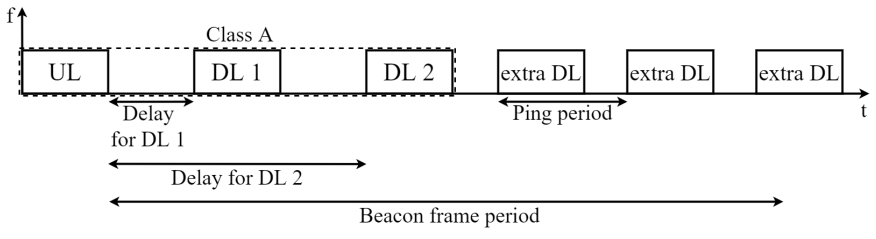

Fig. 13. LoRaWAN Class B

Class C stands for Continuous, meaning that an ED implementing Class $\mathrm{C}$ approach is constantly waiting for DL transmissions between its UL transmissions. Class $\mathrm{C}$ adds no extra MAC commands and any of Class A MAC commands have to be transmitted in the related available windows and not in the continuous reception window. Should an ambiguity occur with a Class $\mathrm{A}$ receive window and Class $\mathrm{C}$ continuous reception in a Class C-enabled ED, Class A DL messages are prioritized. If a Class A successful reception took place in the $1^{\text {st }}$ window, no $2^{\text {nd }}$ window is opened, as mentioned earlier, meaning that the continuous DL window will take its place. The transmission scheme can be seen in Fig.14.

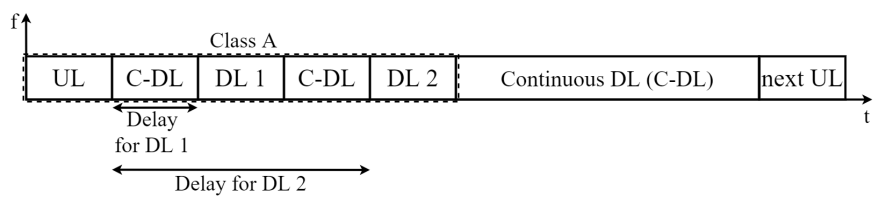

Fig. 14. LoRaWAN Class C

Naturally, Class A is the best option power-wise, but forces the NS to wait for the next UL transmission if it can't be served by the two open windows. On the other hand, Class $\mathrm{C}$ reduces latency for NS to ED communication and practically communication ceases being UL-initiated (except for the first transmission which must be a UL), but ED has to be ready to receive DL messages all the time, resulting in greater energy demands. Class B stands in the middle point of Class A and Class C.

\section{B. LoRaWAN messages \& node activation}

Using the functionality offered by the lower LoRa PHY layer, LoRaWAN formats its packets to support message exchange between EDs and GW. The format of LoRa PHY layer packet is discussed on the corresponding section. The format of the LoRaWAN MAC frame can be seen in Fig.15.

\begin{tabular}{|l|l|}
\hline MAC Payload & MIC \\
\hline
\end{tabular}

Fig. 15. LoRaWAN frame format

The frame has three main fields. $M H D R$ field is the header field for MAC layer, the payload of MAC layer resides inside the MAC Payload field and the MIC field stands for Message Integrity Code. In the latter field, the MIC is calculated using the fields of the frame and AES CMAC algorithm. Considering the MAC Payload field, it may either include the payload of a message, Join-Request/Join-Accept messages for EDs trying 


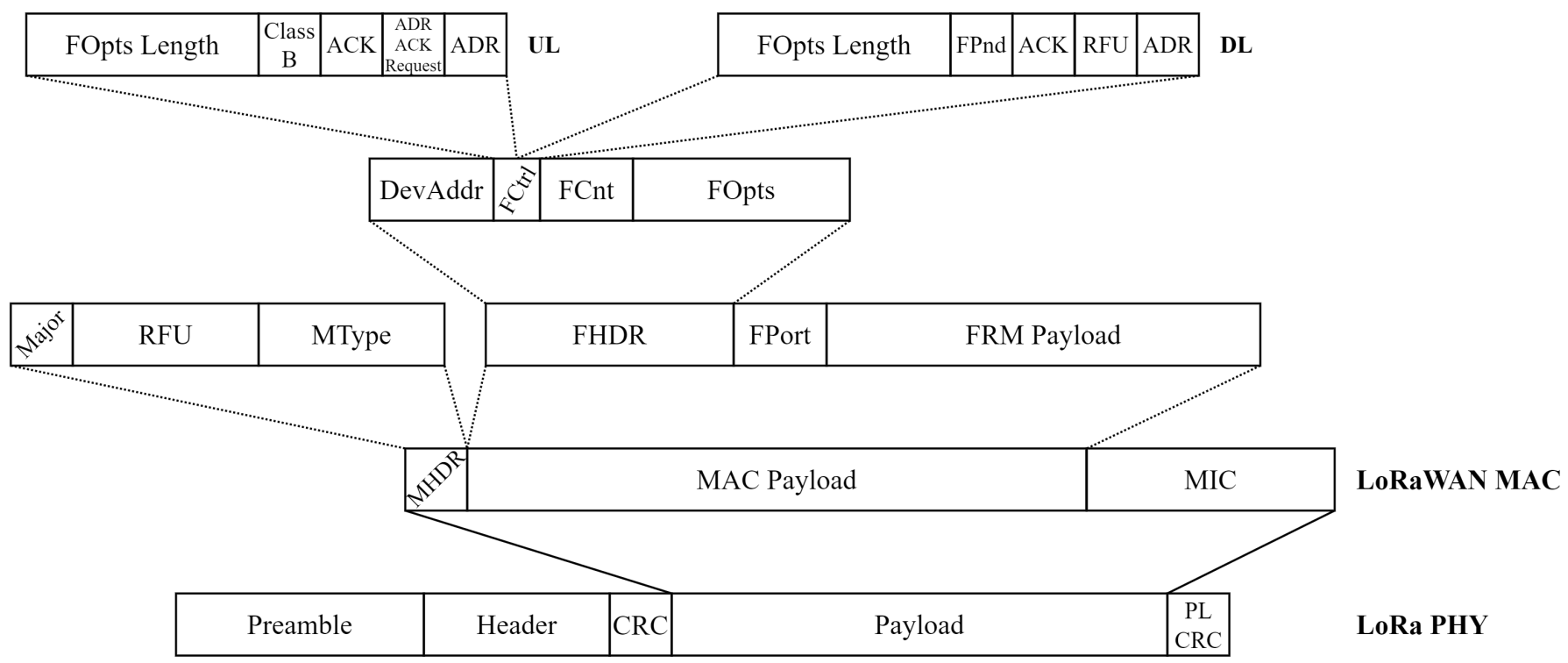

Fig. 16. Packet encapsulation in LoRaWAN

to join the network or other MAC commands. In Fig.16, a deeper view of packet encapsulation process in LoRaWAN is given $^{3}$.

An extensive analysis of each field is not in the scope of this paper. The interested reader can find more in official documentation [56]. In this context, we focus on the 3-bit MType field in an effort to present the different types of messages supported by the protocol. In LoRaWAN, 8 types of messages are supported as shown in TABLEIV.

TABLE IV

MESSAGE TYPES SUPPORTED BY LORAWAN

\begin{tabular}{|c|c|}
\hline MType field & Message Description \\
\hline 000 & Join-Request \\
\hline 001 & Join-Accept \\
\hline 010 & Unconfirmed Data Up \\
\hline 011 & Unconfirmed Data Down \\
\hline 100 & Confirmed Data Up \\
\hline 101 & Confirmed Data Down \\
\hline 110 & Reserved (v1.0.x) / Rejoin-Request (v1.1) \\
\hline 111 & Proprietary \\
\hline
\end{tabular}

Messages with an MType field value of 010 and 011 represent UL and DL traffic with no ACK respectively. An MType field of 100 and 101 is used for UL and DL messages that require ACK. 111 value is proprietary, in a sense that it can be used to describe messages that do not follow the standard format. Messages 000, 001 and $110^{4}$ are used for activation of ED via OTAA process.

In LoRaWAN two activation processes are distinguished: OTAA and ABP. In OTAA, ED has to first be "personalized", meaning that a device ID, called DevEUI, a join ID, called JoinEUI $I^{5}$ and an application key, called AppKey have to be

\footnotetext{
${ }^{3}$ In DL messages the field of Payload CRC (PL CRC) does not exist.

${ }^{4}$ In Specification version 1.0.x, 110 value is described as RFU, meaning Reserved for Future Use. In Specification version 1.1, 110 value is used for Rejoin-Request messages by the ED.

${ }^{5}$ To avoid confusion, in older versions of the protocol JoinEUI is mentioned as AppEUI. As of 1.0.4 and 1.1, versions the former nomenclature is adapted.
}

defined. With these values set, ED can send a Join-Request message with DevEUI and JoinEUI values included in it along with a DevNonce. Should the network accept this request, NS sends a Join-Accept message, consisted of the fields of JoinNonce ${ }^{6}$, NetID, DevAddr, DL settings, Rx delay and optionally a channel list field relevant to transmission regulations in each area. With these fields and the AppKey value, the NwkSKey and AppSKey can be derived using AES 128. These keys are used for security purposes as described at the end of this LoRaWAN section. The OTAA process is described in Fig.17.

Considering ABP procedure, the join accept/request messages are bypassed and the user stores directly to ED the required keys and device address. As a method, it might seem simpler, but has two serious drawbacks. Firstly, the ED is associated with the specific network through which has been activated. Secondly, the keys are derived by the user, which involves the danger of non-careful selection of them, thus risking the security of the device or even of the entire $\mathrm{WSN}^{7}$.

After successful activation of the device with either of these two methods, DevAddr, NwkSKey and AppSKey are set to ED.

\section{Security in LoRaWAN}

In LoRaWAN, security is provided via the the use of two of the keys described in the previous subsection; namely NwkSKey and AppSKey. NwkSKey stands for Network Session Key, although it refers to security aspects in LoRaWAN MAC. NwkSKey is used by ED and NS for encryption and decryption processes of the LoRaWAN MAC payload, as well as for ensuring integrity ( $M I C$ field in frame format). On the other hand, AppSKey, an abbreviation of Application Session Key,

\footnotetext{
${ }^{6} \mathrm{To}$ avoid confusion, in older versions of the protocol JoinNonce is mentioned as AppNonce. As of 1.0.4 and 1.1 versions, the former nomenclature is adapted.

${ }^{7}$ Due to the importance of that risk, [56] emphasizes the necessity of proper key derivation.
} 


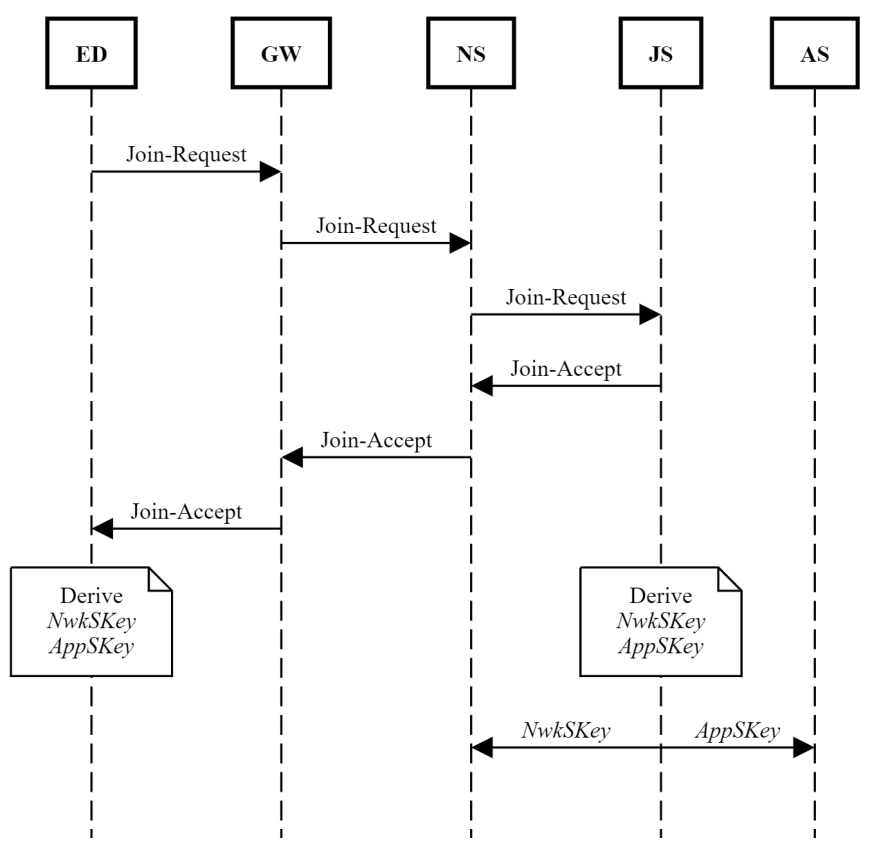

Fig. 17. OTAA sequence diagram (ED: End Device, GW: Gateway, NS: Network Server, JS: Joint Server, AS: Application Server)

is used for encryption and decryption processes regarding the APP payload.

It can be inferred that encryption is guaranteed end to end, but that is not the case with integrity. Encryption is vital for network's sustainability, due to the broadcasting nature of UL transmissions that can be captured by any GW in proximity; even GWs that are not part of the system. On the topic of integrity, NS is considered as a trusted entity in general, however a user may implement additional confidentiality and integrity mechanisms for the applications. A reference on the topic of system's security in a LoRaWAN deployment can be found in [85]. Even broader discussions encompassing the entirety of IoT field can be found in [86], [87].

\section{AN OVERVIEW OF THE LORA MOdulation}

In contrast to LoRaWAN, LoRa is a proprietary PHY layer modulation being developed by Semtech. Its definition follows:

"LoRa ${ }^{\circledR}$ is a modulation technique, suitable for interconnecting things with energy constraints in long range wireless environments, with low needs of data rate."

LoRa development as a technology started from a French company called Cycleo and eventually was patented by O. B. A. Seller and N. Sornin [88], [89] with Semtech assignee. Cycleo was acquired by the USA-based company named Semtech, in 2012. Since then, LoRa is maintained and developed by Semtech. A brief history of LoRa can be found in [90].

\section{A. Implementation of LoRa in chipsets}

Semtech is responsible for the development of chipsets that are capable of interconnecting EDs with GWs, via an established LoRa link. A number of chipsets are in use today inside LoRa EDs; families of SX1261/62/68, SX1272/73, SX1276/77/78/79 are considered the most common, LLCC68 is the preferable option in smart home applications due to lower maximum link budget and worse receiver sensitivity and SX1280/81 is a chipset that can offer LoRa connectivity in the $2.4 \mathrm{GHz}$ band. These chipsets can be found inside LoRa modules built by other hardware manufacturers, like Microchip Technology (RN2483 is one of the most widely used modules and is based on SX1276/77/78/79 family), Murata Manufacturing (its CMWX1ZZABZ-078/-093 module uses SX1276 chipset), STMicroelectronics, HopeRF, Gemtek, AcSiP and others. A comparison among Semtech's chipsets can be found in TABLEV. Values of TABLEV are nominal, as provided by Semtech.

On the area of GWs, a number of chipsets are also available. SX1250, which works along with SX1302, SX1255/57/58 (frequency: $400 \mathrm{MHz}-510 \mathrm{MHz} / 860 \mathrm{MHz}-1000 \mathrm{MHz} / 779 \mathrm{MHz}-$ 787MHz), SX1301, SX1302, SX1308 are common options. Major companies develop their own GWs based on these chipsets, like Cisco (GW based on SX1301), Kerlink, Multitech and others who are operating in this field.

\section{B. Performance metrics of a LoRa link}

The main metrics regarding a LoRa link are the maximum achievable bit rate and the maximum range supported. The former can be calculated by taking into account 3 of the key performance factors (SF, CR, BW), while the latter is depicted in receiver's sensitivity or link budget equations and is related to the $4^{\text {th }}$ factor (TP). For the interested reader, Appendix A provides a further mathematical definition of $\mathrm{BW}, \mathrm{SF}$, chirp and chip in the context of LoRa. The bit rate of a LoRa link is given by the formula:

$$
R_{b_{(b / s)}}=S F * \text { RateCode } * \frac{B W_{(H z)}}{2^{S F}}
$$

The fact that $R_{b}$ is proportional to $S F$ linearly and inversely proportional with it in an exponential way, leads to the conclusion that an increase of $S F$ will have a negative impact on data rate. On the other hand, regarding the receiver's sensitivity we have the following formula:

$$
S_{(d B m)}=-174+10 * \log _{10}\left(B W_{(H z)}\right)+N F_{(d B)}+S N R_{(d B)}
$$

where $B W$ is the bandwidth in $\mathrm{Hz}, N F$ is the receiver Noise Figure in $\mathrm{dB}$ (which for certain LoRa implementations can be fixed at $6 \mathrm{~dB}$ ) and $S N R$ is the signal to noise ratio threshold for a given spreading factor in $\mathrm{dB}$.

The opposite is the occasion with receiver's sensitivity. An increase of $S F$ will lead to a lower receiver sensitivity threshold, leading to a receiver being able to decode signals received with less power. The relationship between sensitivity and SF is not clear in equation 2, but can be derived when examining equation 3. In LoRa, $6 \mathrm{SFs}$ are in use, from SF7 to SF12 and the selection of SF defines the value of SNR threshold. Interestingly, all the nominal values of SNR threshold are negative, which reveals another benefit of LoRa: the fact that receiver can decode signals even below the noise floor.

$$
S N R_{\text {threshold }_{(d B)}}=-7.5+(7-S F) * 2.5
$$


TABLE V

COMPARISON OF LORA CHIPSETS BY SEMTECH

\begin{tabular}{|c|c|c|c|c|c|c|}
\hline Chipset & $\begin{array}{l}\text { Frequency } \\
\quad(\mathrm{MHz})\end{array}$ & $\begin{array}{c}\text { Maximum } \\
\text { Transmission } \\
\text { Power }(\mathrm{dBm})\end{array}$ & $\begin{array}{c}\text { Rx Sensitivity } \\
(\mathrm{dBm})\end{array}$ & $\begin{array}{l}\text { Maximum Link } \\
\text { Budget (dB) }\end{array}$ & $\begin{array}{c}\text { Supported } \\
\text { Modulation }\end{array}$ & Bit Rate $(\mathrm{kb} / \mathbf{s})$ \\
\hline LLCC68 & $150-960$ & 22 & -129 & 151 & $\begin{array}{l}\text { FSK, GFSK, MSK, } \\
\text { GMSK, LoRa }\end{array}$ & $\begin{array}{l}\text { Programmable bit } \\
\text { rate from } 1.76 \text { up } \\
\text { to } 62.5 \mathrm{LoRa} \\
\text { and } 300 \mathrm{FSK}\end{array}$ \\
\hline SX1261/62/68 & $150-960$ & 22 & -148 & 170 & $\begin{array}{l}\text { FSK, GFSK, MSK, } \\
\text { GMSK, LoRa }\end{array}$ & $\begin{array}{l}\text { Programmable bit } \\
\text { rate up to } 62.5 \\
\text { LoRa and } 300 \text { FSK }\end{array}$ \\
\hline SX1272/73 & $860-1000$ & 20 & -137 & 157 & $\begin{array}{l}\text { FSK, GFSK, MSK, } \\
\text { GMSK, LoRa, OOK }\end{array}$ & $\begin{array}{l}\text { Programmable bit } \\
\text { rate up to } 300\end{array}$ \\
\hline SX1276/77/78/79 & $\begin{array}{c}137-1020 \\
137-525 \text { (for SX1278) }\end{array}$ & 20 & -148 & 168 & $\begin{array}{l}\text { FSK, GFSK, MSK, } \\
\text { GMSK, LoRa, OOK }\end{array}$ & $\begin{array}{l}\text { Programmable bit } \\
\text { rate up to } 300\end{array}$ \\
\hline SX1280/81 & 2400 & 12.5 & -132 & 144.5 & $\begin{array}{l}\text { LoRa, FLRC, } \\
\text { (G)FSK }\end{array}$ & Programmable \\
\hline
\end{tabular}

$$
P_{R x(d B m)}=P_{T x(d B m)}+\text { SystemGains }_{(d B)}-\text { SystemLosses }_{(d B)}-\text { ChannelLosses }_{(d B)}-M_{(d B)}
$$

The relationship between sensitivity and SF has an impact on the potential range of the system, since a lower sensitivity threshold permits an ED to communicate with a more distant GW. The trade-off of range and bit rate can be quantified through equations 1-3.

Moreover, link budget can be defined by calculating the expected power on the receiver's end as described in equation 4, where $P_{R x}$ is the expected power in receiver's end in $\mathrm{dBm}$, $P_{T x}$ is the transmitted power in $\mathrm{dBm}$, SystemGains is the gain in link's ends in $\mathrm{dB}$ (practically $\mathrm{Tx}$ and $\mathrm{Rx}$ antenna gains), SystemLosses is the loss in link's ends in $\mathrm{dB}$ (practically loss due to connection of antennas to radio modules via e.g., wiring), ChannelLosses is the loss in link's channel in $\mathrm{dB}$ (practically referring to all the phenomena affecting wireless signal transmission, i.e., reflection, refraction, diffraction, scattering and absorption) and $M$ is the fading margin in $\mathrm{dB}$. We can also calculate the link budget of a link with respect to transmitted power and receiver sensitivity, as follows:

$$
P_{R x_{(d B m)}}=P_{T x_{(d B m)}}-S_{(d B m)}
$$

A visualization of the inversely proportional and proportional relationship between $S F$ and bit rate, and $S F$ and range respectively is given in Fig.18. Naturally, an increase of $S F$ results in linear increase of system's range, whereas the decrease of supported data rate is exponential.

Ending this quantitative section, we examine the regulations of ISM transmission by exploring the concepts of ToA and duty cycle. LoRa as a technology utilizes the ISM bands in a region-based scheme, although chipsets that support globally free bands like the $2.4 \mathrm{GHz}$ exist. Due to the use of these bands, LoRa links have to comply with a number of regulations:

- change transmission channel in a pseudorandom fashion in every transmission,

- respect the maximum duty cycle (or dwell time) and the maximum transmitted power (Effective Isotropic Radiated Power (EIRP) and Effective Radiated Power (ERP)),

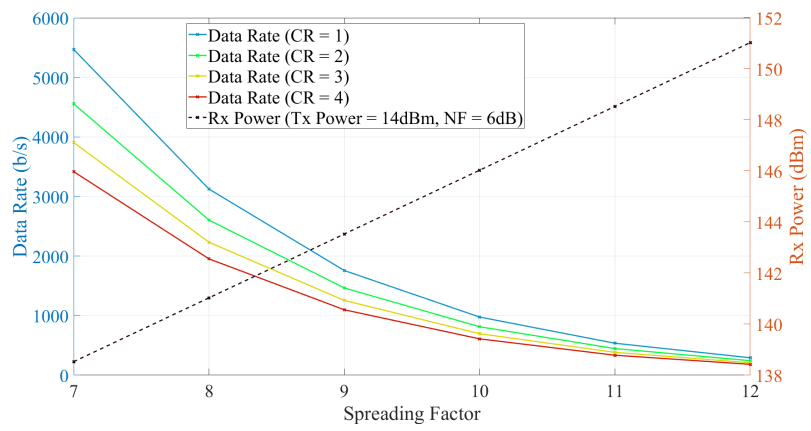

Fig. 18. Trade off between data rate and range $(\mathrm{BW}=125 \mathrm{kHz})$

Compliance to these regulations is necessary, since a plethora of technologies reside in ISM bands. Focusing on European region, the duty cycle imposes the maximum time allowed to transmit, with common maximum values being $0.1 \%, 1 \%$ and $10 \%$. In North American region, dwell time is the regulated value.

The structure of a LoRa PHY packet can be viewed in Fig.19.

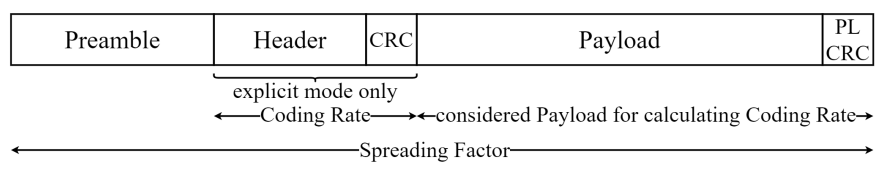

Fig. 19. LoRa packet format [54]

A Preamble being transmitted in the form of a number of up-chirps followed by two and a quarter down-chirps indicates the start of the packet. After that, the Header and CRC fields are included if explicit mode is used. The size of these fields depends on CR, and a typical rate code of $\frac{4}{5}$ with an 8Bytes Payload plus Payload CRC field size, would result on a combined size of Header and CRC of 2Bytes. In the example of Fig.19 packet formatting, we observe that the size 


$$
\begin{gathered}
T_{\text {payload }}=T_{S} *\left\{S W+\max \left[\operatorname{ceil}\left(\frac{8 * P L-4 * S F+28+16 * C R C-20 * H}{4 *(S F-2 * D E)}\right) *(C R+4), 0\right]\right\} \\
T o A=\frac{2^{S F}}{B W_{(H z)}} *\left\{\left\{n_{\text {preamble }}+4.25\right\}+\left\{S W+\max \left[\operatorname{ceil}\left(\frac{8 * P L-4 * S F+28+16 * C R C-20 * H}{4 *(S F-2 * D E)}\right) *(C R+4), 0\right]\right\}\right\}
\end{gathered}
$$

of Header and CRC fields are half the size of Payload and Payload CRC, indicating a CR of 4. The Payload field includes the payload information that the ED broadcasts, assuming a UL transmission.

Based on Fig.19, the transmission time of a PHY layer packet can be split in the time needed for preamble and the time needed for payload. We can easily infer that ToA will be:

$$
\text { ToA }=T_{\text {preamble }}+T_{\text {payload }}
$$

The formula for calculating $T_{\text {preamble }}$ is given by equation 7 :

$$
T_{\text {preamble }}=\left(n_{\text {preamble }}+4.25\right) * T_{s}
$$

where $n_{\text {preamble }}$ is fixed for EU868 band in 8 symbols and $T_{s}$ is transmission time for one symbol, as expressed in equation 8 and derived in Appendix A (equation A5):

$$
T_{S}=\frac{2^{S F}}{B W_{(H z)}}
$$

On the other hand, the time to transmit payload is given by equation 9, where $T_{s}$ is the time for a symbol transmission in $\mathrm{s}$, $S W$ is synchronization word and has a fixed value of $8, P L$ is the number of payload bytes, $S F$ is the spreading factor, $C R C$ is a one-bit flag that can be set at 1 if the used protocol above is LoRaWAN, $H$ is the header type as discussed in Fig.19 (0: explicit, 1: implicit), $D E$ is a one bit flag that notifies for low data rate optimization when SF11 or SF12 is used (0: high data rate, 1: low data rate) and $C R$ is coding rate.

Finally, equation 10 provides the total ToA, taking into consideration equations $6,7,8$ and 9 .

Having regulated the percentage of time that an ED can transmit, we clearly see a pattern being shaped in UL transmissions. A node can transmit, then it has to wait for at least a minimum amount of time during which it may or may not receive DL messages and after that it can re-transmit. We refer to this waiting time as $T_{\text {interval. }}$ If we examine the relationship between $T o A$ and $T_{\text {interval }}$ and taking into account the regulation about duty cycle, we have:

$$
T_{\text {interval }}=\frac{\text { ToA }}{\text { DutyCycle }}-T o A
$$

This results in $T_{\text {interval }}$ being orders of magnitude greater than ToA.

\section{RESEARCH ON LORA/LORAWAN SYSTEMS}

There are various open research topics related to the implementation, usage, and optimization of the LoRa/LoRaWAN networks [91], [92]. The major ones are listed below:

- Power consumption, energy management and lifetime of the system (e.g., [40])
- Range management and link-level behavior (e.g., [14])

- Network performance validation and evaluation metrics (e.g., [12])

- Multiple Access (e.g., [58])

- Security in PHY, MAC and NET layers (e.g., [93])

- ADR algorithm (e.g., [77])

- ED mobility support and roaming (e.g., [78])

- Optimal GW placement and localization challenges (e.g., [94])

- Data-based decision making by incorporating Machine Learning (ML) (e.g., [95])

- Meeting recent advancements from Information Technology (IT) and Telco domains, e.g., Software-Defined Networking (SDN), Blockchain and network slicing (e.g., [96])

The research work on addressing challenges related to the above-listed topics includes approaches for all the well-

\begin{tabular}{|c|c|}
\hline $\begin{array}{c}\text { Target of the } \\
\text { Survey and Tutorial }\end{array}$ & References \\
\hline IoT & $\begin{array}{l}\text { [2] (full overview), [5] (definitions and chara- } \\
\text { cterization), [10] (IIoT full overview), [38] } \\
\text { (full overview with focus on specific verti- } \\
\text { cal), [87] (security aspects), [99] (Block- } \\
\text { chain applicability), [100] (Blockchain appli- } \\
\text { cability), [101] (technologies \& verticals), } \\
\text { [102] (DT full overview), [103] (DT full } \\
\text { overview), [104] (DT full overview), [105] } \\
\text { (DT full overview), [106] (IIoT edge com- } \\
\text { puting), [107] (IIoT security aspects) }\end{array}$ \\
\hline WSN & $\begin{array}{c}\text { [24] (full overview) } \\
\text { [97] (WSN evaluation methodology) }\end{array}$ \\
\hline LPWAN & $\begin{array}{l}\text { [13] (LoRa \& Sigfox comparison), [15] (com- } \\
\text { parison, verticals, research challenges), [16] } \\
\text { (design considerations, comparison, re- } \\
\text { search challenges), [17] (LoRa \& NB-IoT com- } \\
\text { parison), [18] (overview, comparison, research } \\
\text { challenges), [19] (Sigfox, LoRaWAN \& NB- } \\
\text { IoT comparison), [20] (LoRaWAN, DASH7 \& } \\
\text { NB-IoT comparison and mobility support), } \\
\text { [21] (verticals, comparison), [22] (full over- } \\
\text { view), [45] (ISM LPWAN security aspects) }\end{array}$ \\
\hline LoRa/LoRaWAN & $\begin{array}{l}\text { [79] (ADR algorithm), [82] (LR-FHSS), [85] } \\
\text { (LoRaWAN v1.1 security aspects), [91] (LoRa } \\
\text { research challenges), [92] (LoRaWAN full over- } \\
\text { view), [98] (LoRa research challenges), [108] } \\
\text { (full overview), [109] (LoRaWAN full over- } \\
\text { view), [110] (LoRaWAN simulators) }\end{array}$ \\
\hline
\end{tabular}
known steps in research methodology [97], [98]; namely: mathematical analysis, simulation study, and real-life system implementation.

TABLE VI

LIST OF RELATED SURVEYS

A set of surveys has also been published ([2], [5], [10], [13], [15]-[22], [24], [38], [45], [79], [82], [85], [87], 
[91], [92], [97]-[110]) examining IoT, WSN, LPWAN and LoRa/LoRaWAN from various perspectives, one of which is the research challenges. This set of publications is summarized in TABLEVI. Indicative research contributions that target specific problems and challenges are studied in the following subsections.

\section{A. Analytical approaches}

The first step towards the study of LoRa/LoRaWAN networks is to evaluate the performance and the behavior of the system using mathematical tools. Such tools include but not limit to methods like: calculation of performance metrics in probabilistic manner and comparison with well-known results (e.g., performance of LoRaWAN throughput in comparison with protocols like ALOHA, Carrier Sense Multiple Access (CSMA) and variations of them), modeling of the system with stochastic tools under various scenarios and assumptions (e.g., Poisson process, Markov process, stochastic geometry) and others.

In [60], the authors provide an analysis for the probability of successful UL transmissions when taking into account co$\mathrm{SF}$ interference, inter-SF interference or both of them. The imperfect orthogonality of SF is proven to affect UL LoRa links. In [78], a statistical approach to the effect of SF selection and different levels of mobility in ADR is presented. The authors gather UL data based on a real testbed and with these data they perform a Kruskal-Wallis non-parametric test. Their analysis exposes a high correlation between SF selection dictated by Blind ADR and performance, especially in lower mobility experiments ( $\mathrm{p}$-value is always at least at 2 significant digits between increment pairs of SFs).

The modeling of interference in LoRa networks either in the form of inter-SF or in the form of intra-SF is of great interest. For the latter case, the authors in [111] derive an interference model based on their proposal for a coherent LoRa transceiver. They calculate the necessary expressions for Symbol Error Rate (SER) and Frame Error Rate (FER) utilizing the Discrete Fourier Transform (DFT). Due to heavy computational complexity, they also use Q-functions for approximation purposes and later they compare their theoretical model with simulation results for the typical non-coherent transceiver and their proposed coherent one. In [112] the performance of CSS is examined in regard to SF orthogonality with the derivation of closed form expressions, whereas in [113] the author gives insights in LoRa modulation examining it by a quantitative signal processing perspective, while also coming up with an optimal receiver based on Fast Fourier Transform (FFT). Results are drawn by comparing the optimal receiver with the typical FSK modulation in terms of BER versus SNR.

In [114], the authors propose their own MAC solution in order to alleviate the low Packet Delivery Ratio (PDR) problem of pure ALOHA-based approach in LoRaWAN. They name their protocol Lightweight Carrier Sensing (LCS) and evaluate it by comparing it with pure ALOHA in a threefold way: mathematical approach, simulation and small scale implementation. LCS has two main features: i) a hardware level "hearing" mechanism, which is called Channel Activity Detection (CAD), and ii) a packet drop mechanism when the channel is sensed busy instead of random backoff. For the initial evaluation part, they derive a probability model for the PDR of their mechanism and compare it with the known PDR of pure ALOHA. The results showcase a considerable improvement in PDR for the proposed mechanism over pure ALOHA, especially for large numbers of EDs. Another MAC suggestion can be found in [65], where the alternative of slotted ALOHA is discussed. The proposed protocol is based in FM-RDS broadcasting for time dissemination and the authors explore the applicability of the protocol in all 3 possible research steps. For the mathematical approach, they derive closed form expressions for Type-A and Type-B uncertainty of the transmission time of packets, which is caused due to a packet moving among the stacks of the entire protocol stack inside a node.

In [115] the authors present an analytical approach to the topic of LoRa networks scalability based on stochastic geometry. In essence, their efforts target the derivation of closed form expressions for outage probability regarding to two events: i) the event of a packet having SNR below the required threshold in the receiver, and ii) the event of less than $6 \mathrm{~dB}$ difference between the interested signal and the interferers (practically capture effect). For these two events the probabilities are calculated and the results are further validated with Monte Carlo simulations. In [116], the OTAA activation mechanism of Class A EDs in LoRaWAN is evaluated. The authors formulate the problem as a discrete time Markov Chain (MC) and derive the transition probabilities for all the possible transitions between states, in an effort to estimate the energy that an ED consumes when it tries to get activated and the delay that exists until successful activation.

Reference [117] examines the topic of energy harvesting in the more general context of WSNs, providing analytical expressions for power consumption and throughput of the system when realistic assumptions are taken, such as battery inefficiencies. To provide these analytical expressions, the authors formulate the problem as a Markov Decision Process (MDP). In [118], the same problem of energy harvesting is discussed, but this time in specific for LoRaWAN systems. An MC model is presented and results are drawn regarding PDR for Class A EDs. These results are later compared to simulation experiments. Energy issues are also discussed in [119], in which the authors derive analytical expressions for energy consumption, ED lifetime and energy cost for different types of UL transmissions, and propose their own energy models. Their analysis is based on measurements from an experimental testbed, as described later. The same approach can be found in [80]; a derivation of analytical expressions for energy consumption. These expressions are validated through simulations and the results are discussed further in the corresponding simulation studies subsection that follows.

A resource allocation framework based on a Network Slicing scheme orchestrated by a Deep Federated Reinforcement Learning (DFRL) system architecture is presented in [95]. The authors focus their efforts on providing different network slices for Industrial IoT (IIoT) use cases. The slices are 
allocated thanks to GWs acting as agents, TP and SF acting as environmental parameters and QoS being the reward that needs to be maximized, according to the typical agent-environment interaction in reinforcement learning problems [120]. The algorithm that is developed maximizes the QoS reward, which can be interpreted as slice delay, energy consumption, throughput and Packer Loss Rate (PLR).

Network slicing for resource allocation purposes is also considered in [96]. The authors distinguish application scenarios according to their QoS needs in 3 slices and formulate the problem of network slicing for each ED with a game theoretic approach. The slicing is performed in a distributed way at the GW level. 3 games are designed: the first one between GWs for cell edge EDs assignment, the second for physical channel reservation for each slice in each $\mathrm{GW}$ and the final for channel assigning in each ED of each slice for each GW. Both the former and the latter game are designed as matching theory problems (one-to-many and one-to-one respectively), whereas the second game is formulated as a bankruptcy game. Their model is further validated via simulations as explained in the next subsection.

\section{B. Simulation studies}

Simulation-based research dominates the area of WSNs and consequently LPWANs, due to simulators capability to support experimentation with crowded networks. To this end, numerous simulation environments can be utilized, with main considerations being the ones presented in a comprehensive overview in Section IX later. This subsection presents indicative research work conducted with the aid of LoRa/LoRaWAN dedicated simulators as well as other, yet more generic network simulation frameworks like OMNeT++ and NS-3.

In [12], the authors develop a simulator to compare LoRaWAN capacity with pure ALOHA, with the results depicting an equivalent performance of the two protocols. Further tests they perform, are based on a testbed as described in the corresponding section that follows. In [40], a comparative study is provided for several LPWAN solutions, with LoRa being among them. The study includes simulations conducted in Matlab with realistic assumptions, in an effort to estimate the performance of all of these solutions in terms of coverage and energy consumption and consequently the impact on EDs' lifetime. In [58], the authors quantify the throughput of pure ALOHA, which is supposed to be the multiple access mechanism performed by LoRaWAN. They also propose a slotted ALOHA mechanism built in top of LoRaWAN protocol and based in a synchronization procedure, in an effort to improve throughput. To validate their results they conduct Matlab simulations and real testbed experiments as described later. In [59], the authors develop two endpoints of a LoRa transmission (a modulator and a demodulator) in Matlab, in an attempt to estimate the impact of SF's imperfect orthogonality in BER and Packet Error Rate (PER) with respect to Signal to Interference Ratio (SIR), for various combinations of useful transmissions' SF and interferers' SF.

The analytical results provided in [60] are further validated with simulations that extract system throughput under different conditions. Simulation details are given, but no further elaboration on the simulation environment is available. In [80], the authors propose an energy model for LoRaWAN EDs and validate their model via simulations. Again, simulation details regarding the environment are not given, however the parameters of the tested scenarios are provided. Results follow intuition regarding the effects of $\mathrm{SF}$ and $\mathrm{CR}$ selection on the energy consumption, the relationship between range and energy consumption and the most demanding tasks energywise for an ED. Regarding the topic of multiple access, a proposal can be found in [62], in which a variant of CSMA/Collision Avoidance (CSMA/CA) is examined as an option, instead of pure ALOHA that LoRaWAN is thought to be. Experiments in crowded $\mathrm{OMNeT}++$ simulations are performed, which reveal a sizeable improvement in successful packet reception and consequently system's throughput.

In [55] a discrete event simulator based on Python is described, called LoRaSim. The simulator is used to obtain results that are related to scalability potential of a LoRa system and Data Extraction Rate (DER); a metric that compares the total number of received messages to the total number of sent messages. The discrete event simulator of LoRaSim is used and modified extensively and one such example is [63], where a new MAC layer approach is examined, called MAC on Time (MoT). The authors compare their proposed protocol, which is a combination of TDMA and contentionbased schemes, with the ALOHA-based LoRaWAN protocol. The results reveal a superiority of MoT both in throughput and in latency performance metrics. Moreover, in [121] the same simulator is used for DER measurements and the impact that directional antennas on EDs and an increase on the number of GWs may have on the topic of interference scaling. In [122] the LoRaSim simulator is extended with MAC layer considerations, as described in the corresponding subsection of the simulator (Section IX). Results include evaluation of successful transmissions as goodput and energy consumption, while insights on the impact of ACK packets in LoRaWAN systems scaling are given.

The same extended simulator is used to evaluate the ADR algorithm described in LoRaWAN 1.1. The study in [77] reveals a slow convergence of the algorithm, which deteriorates the energy consumption and PDR. A further extension, called LoRaEnergySim, is provided in [123], where realistic assumptions are taken regarding energy consumption and results are drawn for both energy and DER. The same simulator (LoRaEnergySim) with the necessary modifications [124] is used to evaluate the proposed slotted ALOHA MAC described in [65]. Results depict a clear improvement in both PDR and energy efficiency, especially when high SFs are used and/or payload length is long. For the combination of SF7 and small packets, pure ALOHA seems the preferable option, at least energy-wise.

LoRa-MAB is another SimPy-based discrete event simulator. The simulator examines the energy consumption per node per successful sent packet and the successful packet transmissions for a proposed algorithm discussed in [125], which focuses on an intelligent distributed resource allocation mechanism that is designed via utilization of Exponential- 
weight for Exploration and Exploitation (EXP3) algorithm to solve the Multi-Armed Bandit (MAB) problem. LoRa-FREE completes the presentation of SimPy-based simulators and is described in [126], where a mechanism of transmitting buffered data in scheduled bulk UL, contrary to transmitting data at the time of generation is investigated. Key metrics include Data Delivery Ratio (DDR) -a metric similar to PDRand energy, but also collision-related metrics and study of ACK-related transmissions. The same simulator is used in order to evaluate Time-slotted LoRaWAN (TS-LoRa) protocol, as discussed in [64].

In [127], the authors present their LoRaWAN simulator implemented in Matlab. After validating the accuracy of their simulator against results of analytical models, they perform several experiments to examine the relation among UL delivery rate (practically PDR), energy consumption, range and number of EDs. In all the occasions, an increment in range or number of EDs affects negatively PDR and energy consumption. Similarly, a stricter duty cycle worsens the DL response rate, however it is more beneficiary for energy consumption. The parameters of the simulations are well provided by the authors.

Another simulator development contribution is described in [128], where a simulator using Java is implemented. With this simulator, the authors perform numerous scalability analysis tests by calculating the PDR with respect to the number of EDs. Their study reveals an unfavorable effect of SF's increase, inter-arrival time decrease and (in the case of high numbers of EDs) payload size increase. CR seems to have no impact on PDR, whereas GWs with multiple channels tend to provide higher PDR, as expected. The capture effect that is present in LoRa transmissions improves the PDR of LoRaWAN when compared to pure ALOHA protocol. QoS requirements are also examined in terms of required PDR for vastly populated LoRa networks. More work with the same simulator includes the further evaluation of the LCS protocol proposed in [114]. The authors perform comparative experiments between LCS and pure ALOHA in terms of energy consumption (based on their proposed energy models) and supported EDs; LCS appears to be superior in both metrics.

OMNeT++ is one of the de-facto simulation environments used extensively in academic research. The framework supports experimentation with a vast type of networks and LoRaWAN simulations are supported by the FLoRa framework. In [129], the authors elaborate on the development of the framework and showcase the potential of the implemented simulator with ADR-related simulations. Three types of experiments are distinguished with ideal path loss, moderate variability of path loss and highly variable path loss for urban and sub-urban environments. ADR clearly improves the performance of the network in terms of PDR and energy consumption when path loss is not taken into consideration, but with path loss present the results depend on the environment and the level of path loss variability. To this end, an improved ADR+ algorithm is proposed, which excels both no-ADR implementation and ADR implementation in the two aforementioned metrics. ns-3 is one of the most widely used simulators in networking research, and regarding the topic of LoRa/LoRaWAN connectivity several references exist. In [57], the required modules for simulating such networks in ns-3 are presented and simulations are developed in order to assess throughput and coverage. [130] and [131] further discuss these modules and provide results regarding PDR. One more research team has extended these ns- 3 modules for simulations focusing on energy consumption, as described in [132]. In [133], another effort on developing the necessary modules for LoRa/LoRaWAN simulations in ns-3 is described providing insights in PDR, whereas a different research group discusses in [134] the development of necessary ns-3 modules for LoRa connectivity and examines energy consumption, PDR and feasibility of CSMA protocol on top of LoRa, when taking into account these two metrics plus the collision rate. Another research topic is the optimization of GW placement, which is presented in [94]. The authors utilize Gap statistics method, K-means clustering and Fuzzy C-means to derive their own model; namely DPLACE. The model is tested and compared with other GW placement methods in ns-3 simulations, showing promising results in terms of Capital Expenditure (CapEx), Operational Expenditure (OpEx), number of required GWs and PDR.

Work [111] has already been mentioned in mathematical approach subsection, however the authors go a step further by putting their proposed interference model for coherent LoRa transceiver to the test via Monte Carlo method. The approximation from Q-function, the coherent transceiver and the non-coherent one are examined, with twofold result: i) the approximations are proven to be extremely close to the simulation results (in many occasions identical), and ii) in terms of SER and FER the coherent transceiver outperforms the non-coherent one. Metrics for SNR and SIR are also provided. Same validation approach for the mathematical analysis of the problem can be found in [115]. The authors confirm their results via Monte Carlo simulations, with the outcomes of their experiments being almost identical to the ones of the numerically calculated outage probabilities.

The mathematical analysis of [116] is also tested via simulations. The proposed MC for the OTAA procedure in LoRaWAN is tested via Scilab simulations with the parameters of the simulations being well described. Results reveal that high channel quality and the existence of multiple available channels have a positive impact in delay and energy metrics, whereas crowded networks are unfavourable to the aforementioned performance factors. Again, the mathematical formulation of the problem is validated through simulations in [112], where the authors set up experiments in Matlab to estimate the performance of CSS in terms of BER and throughput and compare it with BPSK performance, which is used extensively in narrowband solutions.

In [118], simulations are performed based on a discrete event simulator developed in $\mathrm{C}++$ by the authors, in an effort to compare results extracted from an MC formulation of PDR estimation problem in energy harvesting LoRaWAN Class A EDs, as described in the previous subsection. Extending the lifetime of an LPWAN system is an area of extensive research 
and [135] proposes the innovative Long-Lived LoRa MAC protocol to be used in LoRa networks, composed of energy harvesting Class A EDs. The longevity of the system can be further improved via offloading EDs with drained energy to EDs with higher energy load and via parameter selection based on heuristic methods. The proposed model is evaluated via ns3 , in terms of network lifetime and throughput. Results are also compared to a real testbed.

Blockchains are the most widely researched realization of distributed ledgers and their applicability in several networking paradigms is investigated constantly. The intersection of Blockchains protocols with IoT solutions is summarized in [99] and [100]. Related work focusing on LoRa/LoRaWAN connectivity exists both in simulation environments and in real tesbeds. In [93] the authors propose a system architecture with a Blockchain network running in parallel with the typical LoRaWAN system architecture. The Blockchain network is deployed to improve OTAA process and secure the join messages against replay attacks and jamming, thanks to the proposed two-factor authentication mechanism. The mechanism is examined in a virtualized environment with Ethereum client and showcases great results in terms of throughput, however the increase in latency is considerable as the number of EDs rises.

IIoT is one of the main use cases of LoRa/LoRaWAN and a key research area in this frame is resource allocation as mentioned in [95] and described earlier in mathematical approach subsection, where the authors developed a DFRL framework to handle such needs. Their model is tested in Tensorflow (Python) with results being drawn regarding slice delay, energy consumption, throughput and PLR, compared with another network slicing approach and showing considerable improvements in all 4 factors. Network slicing in the context of LoRa/LoRaWAN connectivity is also examined in [96], where the authors perform ns-3 simulations to validate a multi-game theoretic model that they developed for resource allocation. Results are drawn from experiments with different network configuration and slicing strategies and reveal great performance in terms of delay, energy consumption, throughput and PLR.

\section{Real system implementations}

Implementations of IoT systems with the support of LPWANs include: i) small-scale research-oriented testbeds with a few EDs, and ii) large scale deployments serving verticals in the "smart" paradigm.

One of the pioneering works on the topic of system evaluation by means of an experimental testbed is [25] where a LoRa-specific path loss model based on real measurements is derived. The testbed consists of 1 Kerlink GW and 2 SX1272based EDs; one mounted on top of a moving car and one on a boat. The results are promising in terms of range for both cases, assuming lenient requirements for PDR. Regarding the path loss model, it is coined to fit the performed measurements, with different formula parameters for each mobility scenario. Recent publications on the topic of coverage also exist, with [136] examining indoor scenarios using at most 10 SX1272based EDs and at least 2 devices of this type to communicate with 1 GW. Results are drawn for path loss, RSSI, energy consumption and Packet Reception Rate (PRR) (practically PDR).

References [12] and [14] are two more of the earliest works in LPWAN systems. The former work focuses on LoRa connectivity and evaluates some of the specified nominal values. 1 SX1276-based ED and a Cisco GW in an urban environment are used to evaluate the RSSI metric, which is calculated slightly higher than the specified, the coverage in terms of PDR, where the system performs satisfactory and the throughput, which is close to nominal values at the time of that reference's publication. The latter work provides a quick overview of Sigfox and RPMA, whereas sketches in greater detail LoRa/LoRaWAN connectivity. The authors conclude their work with some coverage tests for two use cases. Considering the smart buildings vertical, a successful coverage test is conducted in a 19-store building, placing the GW in the middle point of it and 32 EDs scattered in different places inside of it. Smart city application scenarios are also examined with a Kerlink GW providing connectivity to IMST iM880A-L-based EDs as far as $2 \mathrm{~km}$.

More on the topic of measurement campaigns can be found in [137]. The authors perform extended LoRa measurements in urban and rural scenarios thanks to a combination of both a well-established and an experimental deployment. The entire system is consisted of 3 Kerlink GWs and multiple EDs, but for the needs of the experiments 2 EDs are utilized. Range and coverage are proven feasible even in the $10^{3} \mathrm{~m}$ scale as depicted by RSSI and SNR values, having lenient PER requirements. Part of author's work is also the proposal of a reference architecture which is close to the typical LPWAN case, but with slightly different terminology and a protocol stack, that relies on IEEE 802.15.4 above LoRa and Constrained Application Protocol (CoAP) in APP layer.

As described in the previous subsection, [58] proposes a slotted ALOHA access method on top of LoRaWAN. Apart from Matlab simulations, the authors deploy a real system to perform several tests consisted of a maximum of 24 RFM95W EDs and a GW from Multitech based on a SX1301 and two SX1357 in an indoor environment. The experiments verify the improvement of their slotted ALOHA proposal compared to pure ALOHA. Same is the approach in [59], with the authors validating their Matlab simulation results via the establishment of a link between a USRP and an SX1276-based transceiver. Another MAC consideration is given in [65], where a slotted ALOHA scheme is examined. Mathematical approach and simulation study have already been discussed, but a testbed consisted of 2 SX1272-based ED and 2 Si4703 FM-RDS decoder for time dissemination purposes is also developed.

An innovative approach to the topic of multiple access is given in [61]. The authors propose a new architecture for the access part of the system containing an extra component; namely the Cluster Head (CH). EDs' UL messages can be received by the distant $\mathrm{GW}$ or by the near $\mathrm{CH}$ and then forwarded to $\mathrm{GW}$, whereas the $\mathrm{CH}$ is responsible for distributing DL messages to the EDs of its cluster. To ameliorate the energy consumption of EDs, a TDMA-based scheme is also proposed. Findings from testbed experiments, containing $1 \mathrm{GW}, 1 \mathrm{CH}$ 
and 9 SX1276-based EDs showcase enhancements in PDR, latency and EDs' lifetime.

The deployment presented in [128] is consisted of $3 \mathrm{GWs}$ in urban placement and 4 RFM95W-based (or SX1276-based) EDs with different boards. Experiments are conducted for fixed position indoor, fixed position outdoor and moving EDs, resulting in throughput characterization in PHY layer (similar to theoretical) and APP layer (about 10x decrease). Also, coverage estimation based on PDR is provided, resulting in an optimal $7.5 \mathrm{~km}$ range with $>95 \%$ PDR, an unsatisfactory behavior in indoor scenarios and a non-critical role of velocity in mobility cases, at least not as much as other propagation factors like Line of Sight (LOS). Part of the discussed testbed is also utilized to evaluate the LCS mechanism, presented in [114]. Two of the RFM95W-based EDs and $1 \mathrm{GW}$ were used in an indoor environment to test the transmissions detection precision of the protocol, which proved to be highly accurate.

In [134], the results of the energy-oriented simulation study conducted in [132] are validated thanks to a measurement campaign that includes a Kerlink GW and 4 SX1272 EDs in an outdoor environment with several distances being examined. Energy considerations appear to be a topic of intense research in the area of LPWANs and in [138] the authors deploy a real network in an indoor environment to control a Heating, Ventilation and Air Conditioning (HVAC) system according to room occupation. The network consists of $1 \mathrm{GW}$ and 1 RFM95W-based ED, plus 1 ISM radio ED for comparative reasons. The decision for on/off switching of HVAC is left to a Random Neural Network (RNN) controller, residing in the GW (in the context of that reference the GW is the BS) with the model being trained on a cloud infrastructure. Results are promising in terms of ED's and building's energy consumption, range and path loss, especially when compared to the ISM transceiver.

Energy is a principal consideration in LoRa/LoRaWAN deployments and work in [119] is an example of rigorous analysis of energy consumption in the context of LoRaWAN. The testbed used by the authors is consisted of an SX1272based ED and a Kerlink GW deployed in an indoor environment. Thanks to the taken measurements and analytical formulas numerous results regarding energy consumption, ED's lifetime and energy cost of UL transmissions are drawn. Most surprisingly, the ACK ULs are more energy effective than the non-ACK ones, an outcome that can be attributed to the fact that if a class-A ED receives an ACK in the $1^{\text {st }}$ receive window then it goes to idle state without opening the $2^{\text {nd }}$ receive window. The proposed Long-Lived LoRa protocol is evaluated via simulations as discussed in [135], however a real testbed is also deployed to further investigate the model regarding extended lifetime and system throughput. The testbed is consisted of 1 Raspberry Pi 3 GW based on well-known RAK2245 HAT solution, 15 EDs operating with SX1276 chipset and the open source solution of ChirpStack NS. Both GW and EDs are placed indoor.

Blockchain applicability in LoRa/LoRaWAN is examined not only via simulations but also in testbeds. In [139], the authors propose the HyperLoRa framework, an innovative approach that ameliorates NS workload by exploiting GWs capabilities. A central cloud service that processes all the data is prone to data falsification or loss. With the proposed solution, a Blockchain network at the edge with different ledgers for different types of data (application data at the server-side, OTAA join-request messages at the GW cluster) is built. The network is tested with Hyperledger Fabric (HyperLoRa is the proposed system), 4 GWs (2 SX1255 and 1 SX1301 in each) and 1 ED SX1276, whereas the proliferation is achieved with Locust framework for up to 2000 EDs. Results are promising: amelioration of CPU utilization in NS thanks to distributed message processing between server-side and GW cluster is achieved, improvement in BW utilization between GW and NS is accomplished, whereas throughput is practically the same with or without HyperLoRa. In short, HyperLoRa has close to no HyperLoRa implementation performance but with greater resource utilization.

Further investigation of Blockchain integration in LoRaWAN systems can be found in [140]. The authors are exploring alternative approaches to the topic of data storage and accessibility. To this end, they propose a LoRaWAN-based system, where the data are stored to a Swarm storage space and the access to them is provided via Ethereum smart contacts. Validation of their proposed system is achieved with a testbed that includes 1 Raspberry Pi 2-based ED with Dragino LoRa/GPS Hat, 1 Raspberry Pi 3-based GW with iC880A module, a local proxy server, Swarm storage system and Ethereum client. Results reveal a viable memory utilization for all entities (cloud server-side, GWs and EDs), but throughput appears to be an issue due to EDs proliferation. However, Blockchain's transactions are focused on GWs, which are fewer in numbers in real systems and can aggregate the traffic of multiple EDs.

Finally, in [141] a Blockchain-based architecture for IIoT is proposed. Authors address the problem of data manipulation and the integration of Blockchain protocols to IIoT as a solution to it. The suggested Blockchain IIoT architecture is called BIIT and targets data reliability and improved energy efficiency with little computational overhead. To validate their architecture, the authors set up a testing platform, that includes 1 Arduino-based ED with Dragino LoRa for the LoRa-related experiment and the same ED with Sixfab Cellular IoT Application Shield for the LTE-M-related experiment, a Raspberry Pi acting as a local TTN GW and a Raspberry Pi 3 having a threefold purpose: a Blockchain plugin for TTN, a Blockchain client and a Blockchain miner. The results on packet overhead are very promising, when the number of signed packets is high.

\section{Summary of research points}

Published research in the area of LoRa/LoRaWAN connectivity showcases:

1) The contribution approach may be categorized as analytical, simulation or field deployment. Mathematical analysis is needed to optimize system deployment and operation, field tests are permitted thanks to low CapEx and OpEx, whereas simulations are needed to examine the behavior of the system in terms of ED proliferation.

2) Substantial effort from the research community is allocated on two directions: 
- examine well-known wireless connectivity issues in the context of LoRa/LoRaWAN systems, that affect mainly signal propagation in PHY layer and network access in Data Link layer

- explore the applicability of next generation networks key features and technologies, like ML, SDN, Blockchain and network slicing.

In TABLEVII, recent research work in the area of LoRa/LoRaWAN is classified using as criterion the type of contribution provided. While the references depicted in that table are a meaningful set that can provide the reader with a complete view of the state-of-the-art work, the interested reader is encouraged to check for more work from the authors of the cited publications.

\section{AN OVERVIEW OF LPWAN Simulation TOOLS}

The proliferation of EDs in WSNs dictates the simulation of the interested system to be rolled out, in an effort to estimate its performance, locate any flaws and try to optimize its development. In this context, the available simulators can assist network engineers job, since they allow them to test the system to be deployed, using virtual system components and examining several use cases and scenarios. Among the great advantages of simulation environments is their lack of need to physically deploy devices which might be proved costly in the case of sub-optimal deployment, and their ability to easily scale up the system by just adding virtual nodes.

Focusing on IoT systems and especially on the area of LPWANs and their main representative, LoRa, the target of these simulations is threefold:

1) evaluate the number of devices that can be part of the system, especially in terms of EDs, to estimate the capacity of the system and the margin for upscale,

2) position EDs and GWs in a way that the system's coverage is optimized and

3) use appropriate radio parameters in terms of $\mathrm{SF}, \mathrm{CR}$, $\mathrm{BW}$, TP to estimate the performance of the system.

Other common simulation use cases in the area of IoT include the estimation of EDs' longevity, mobility of EDs and applicability of common WSN algorithms in the examined system.

A number of commercially available and also free and/or open source simulation environments are able to perform the required simulations for a wireless system. Some indicative simulators are [142]:

- The RF design system Planet by Infovista, which allows the design and optimization of wireless cellular networks that have been standardized by 3GPP.

- The software suit Signal Pro by EDX, which includes several modules of widely used networks (e.g., LTE, WiMAX) and provides the necessary tools for RF analysis with the use of propagation models for both indoor and outdoor environments.

- The cellular radiocommunication design tool ASSET Radio by TEOCO, which comes with capabilities of analyzing the coverage of wireless systems, their capacity and the configuration of several parameters in the case of cellular networks.

- The software HTZ Communications by ATDI, that can be used in designing several different wireless systems, like cellular networks, IoT deployments, satellite systems and others.

- A number of software suites by CelPlan, which are applicable in a number of simulation use cases, like design and optimization of an outdoor wireless system, design of a network in an indoor environment, analysis of P2P links and others.

- Several network design tools from Siradel, that include tools for cellular system design, WiFi networks, specialized tools for modeling the signal propagation in $5 \mathrm{G}$ networks and the tool $S_{-} I o T$, which can be used for LPWAN design.

- The Pathloss platform, which can assist the design of radio links that operate in a wide area of spectrum; from $30 \mathrm{MHz}$ up to $100 \mathrm{GHz}$.

Despite the usefulness of these tools, in the rest of this section we will focus on simulation environments that are free to use and support the LoRa modulation and/or the LoRaWAN protocol either in their core codebase or via the use of extensions. The tools that will be shortly examined are: COOJA, TOSSIM, CupCarbon, LoRaWANSim (Matlab), LoRaSim (Java), LoRaSim / MoTSim / LoRaWANSim / LoRaEnergySim / LoRaMACSim (Python), LoRa-MAB, LoRaFREE, OMNeT++ and NS3. Along with the discussion of these simulators, references of published work with the utilization of them are given. An overview of the available open source LoRaWAN simulators can be found in [110]. Additional information on the broader topic of WSN simulators and on the evaluation methodology for such networks is available in [97].

\section{A. COOJA}

Contiki OS Java (COOJA) [143], [144] is a network simulator developed for WSNs. The simulator is used for the study of the behavior of EDs that are called motes and run the Contiki Operating System (OS) [145]. COOJA is an open source simulator and can be found in [146].

Contiki is an OS that finds usage in devices operating under power restrictions. It is an open source software and additional information can be found in the official software repository [147]. Recently, a new branch has been developed, called Contiki NG [148], that is gaining increasing attention and can support several protocols frequently examined in WSNs, like (CoAP), Simple Network Management Protocol (SNMP) and Routing Protocol for Low-Power and Lossy Networks (RPL).

Keeping in mind the advantages of Contiki, it is only natural to be used by numerous EDs that are distinguishing themselves as IoT components and consequently as LPWAN components. Networks that are consisted of such nodes, can be simulated with the aid of COOJA simulator. In the simulator environment, one may analyze the message exchange between EDs and the aggregator, obtain information about the state of the nodes and the communication and also adjust communication parameters. Important aspect of the environment is its 
TABLE VII

A Classification OF THE LORA/LORAWAN RESEARCH WORK

\begin{tabular}{|c|c|c|c|c|c|c|}
\hline \multirow{2}{*}{ Ref. } & \multicolumn{3}{|c|}{ Contribution Approach } & \multicolumn{3}{|c|}{ Evaluation Process } \\
\hline & $\begin{array}{l}\text { Algorithm or } \\
\text { Process Design }\end{array}$ & $\begin{array}{c}\text { Performance } \\
\text { Analysis }\end{array}$ & $\begin{array}{l}\text { System Implementation } \\
\text { (Hardware, Software, } \\
\text { Protocol Development) }\end{array}$ & $\begin{array}{l}\text { Mathematical } \\
\text { Approach }\end{array}$ & Simulation & Experiment \\
\hline$[12]$ & & $\begin{array}{c}\text { RSSI, Capacity } \\
\text { PDR, Throughput }\end{array}$ & & & $\checkmark($ no info) & Urban, $1 \mathrm{ED}, 1 \mathrm{GW}$ \\
\hline [14] & & Coverage & & & & $\begin{array}{l}\text { Indoor \& Urban, } \\
32 \mathrm{EDs}, 1 \mathrm{GW}\end{array}$ \\
\hline$[25]$ & Path loss model & RSSI, Path loss & & & & Urban, 2 EDs, $1 \mathrm{GW}$ \\
\hline [40] & & Coverage, Energy, Lifetime & & & Matlab & \\
\hline$[55]$ & & Scalability, DER & Simulator development & & LoRaSim & D2D link \\
\hline$[57]$ & & Throughput, Coverage & Simulator development & & ns-3 & \\
\hline$[58]$ & $\begin{array}{c}\text { Synchronization } \\
\text { procedure }\end{array}$ & Throughput & $\begin{array}{l}\text { slotted ALOHA on } \\
\text { top of LoRaWAN }\end{array}$ & & Matlab & $\begin{array}{c}\text { Indoor, } \\
24 \mathrm{EDs}, 1 \mathrm{GW}\end{array}$ \\
\hline$[59]$ & & BER to SIR, PER to SIR & & & Matlab & USRP to transceiver \\
\hline$[60]$ & & $\begin{array}{c}\text { Throughput, } \\
\text { Successful UL }\end{array}$ & & $\begin{array}{l}\text { Successful UL } \\
\text { probabilities }\end{array}$ & $\checkmark($ no info) & \\
\hline [61] & & $\begin{array}{l}\text { PDR, Latency, } \\
\text { Lifetime }\end{array}$ & $\begin{array}{l}\text { Network architecture, } \\
\text { TDMA-based MAC }\end{array}$ & & & $\begin{array}{c}\text { Indoor, } 9 \text { EDs, } \\
1 \mathrm{CH}, 1 \mathrm{GW}\end{array}$ \\
\hline [62] & & Throughput & CSMA/CA & & OMNeT++ & \\
\hline$[63]$ & & $\begin{array}{l}\text { Throughput, } \\
\text { Latency }\end{array}$ & $\begin{array}{c}\text { MAC protocol, } \\
\text { Simulator extension }\end{array}$ & & MoTSim & \\
\hline [64] & & PDR, Energy & TS-LoRa & & LoRaFREE & \\
\hline [65] & & PDR, Energy & $\begin{array}{c}\text { slotted ALOHA, } \\
\text { Simulator extension }\end{array}$ & $\begin{array}{l}\text { Type-A, -B } \\
\text { uncertainty }\end{array}$ & $\begin{array}{l}\begin{array}{l}\text { LoRaEnergySim } \\
\text { (LoRaMACSim) }\end{array} \\
\end{array}$ & $\begin{array}{l}2 \text { EDs, } 2 \text { FM- } \\
\text { RDS decoders }\end{array}$ \\
\hline$[77]$ & & ADR & & & LoRaWANSim & \\
\hline$[78]$ & & ADR & & Kruskal-Wallis & & Urban \\
\hline$[80]$ & Energy model & Energy & & Analytical models & $\checkmark($ no info) & \\
\hline [93] & $\begin{array}{l}\text { OTAA two-factor } \\
\text { authentication }\end{array}$ & $\begin{array}{l}\text { Throughput, } \\
\text { Latency }\end{array}$ & $\begin{array}{c}\text { Blockchain-based } \\
\text { architecture }\end{array}$ & & $\begin{array}{c}\text { Ethereum } \\
\text { client }\end{array}$ & \\
\hline [94] & $\begin{array}{l}\mathrm{GW} \text { placement } \\
\text { model }\end{array}$ & $\begin{array}{l}\text { CapEx, OpEx, } \\
\text { PDR, \# of GWs }\end{array}$ & & & ns-3 & \\
\hline [95] & Resource allocation & $\begin{array}{c}\text { Energy, Delay, } \\
\text { Throughput, PLR }\end{array}$ & DFRL architecture & $\begin{array}{c}\text { DFRL } \\
\text { optimization }\end{array}$ & $\begin{array}{l}\text { Tensorflow } \\
\text { (Python) }\end{array}$ & \\
\hline [96] & Resource allocation & $\begin{array}{l}\text { Delay, Energy, } \\
\text { Throughput, PLR }\end{array}$ & $\begin{array}{c}\text { Network slicing } \\
\text { architecture }\end{array}$ & Game theory & ns-3 & \\
\hline [111] & Interference model & $\begin{array}{l}\text { SER, FER, } \\
\text { SNR, SIR }\end{array}$ & $\begin{array}{l}\text { LoRa transceiver with } \\
\text { coherent detection }\end{array}$ & $\begin{array}{c}\text { DFT, } \\
\text { Q-function }\end{array}$ & $\begin{array}{c}\text { Monte Carlo } \\
\text { simulations }\end{array}$ & \\
\hline [112] & & $\begin{array}{l}\text { SF orthogonality, } \\
\text { BER, Throughput }\end{array}$ & & $\begin{array}{l}\text { Closed form } \\
\text { expressions }\end{array}$ & Matlab & \\
\hline [113] & & BER v SNR & Optimal receiver & FFT & & \\
\hline [114] & 2 energy models & PDR, Energy, Scalability & LCS MAC protocol & PDR probabilities & LoRaSim (Java) & Indoor, 2 EDs, $1 \mathrm{GW}$ \\
\hline [115] & & Scalability & & $\begin{array}{l}\text { Stochastic } \\
\text { geometry }\end{array}$ & $\begin{array}{l}\checkmark(\text { no info }) \\
\text { Monte Carlo }\end{array}$ & \\
\hline [116] & & Energy, Delay & & $\mathrm{MC}$ & Scilab & \\
\hline$[117]$ & & Energy, Throughput & & MDP & & \\
\hline$[118]$ & & PDR & & $\mathrm{MC}$ & $\mathrm{C}++$ & \\
\hline [119] & Energy models & Energy, ED lifetime & & Analytical models & & Indoor, $1 \mathrm{ED}, 1 \mathrm{GW}$ \\
\hline [121] & & DER, Scalability & & & LoRaSim & \\
\hline$[122]$ & & Goodput, Energy & Simulator extension & & LoRaWANSim & \\
\hline [123] & & Energy, DER & Simulator extension & & LoRaEnergySim & \\
\hline [125] & Resource allocation & PDR, Energy & Simulator development & & LoRa-MAB & \\
\hline$[126]$ & UL scheduling & DDR, Energy & Simulator development & & LoRaFREE & \\
\hline$[127]$ & & $\begin{array}{c}\text { PDR, Energy, } \\
\text { DL response rate }\end{array}$ & Simulator development & & $\begin{array}{l}\text { LoRaWANSim } \\
\text { (Matlab) }\end{array}$ & \\
\hline [128] & & $\begin{array}{l}\text { Throughput, QoS, } \\
\text { PDR, Coverage }\end{array}$ & Simulator development & & $\begin{array}{c}\text { LoRaSim } \\
\text { (Java) }\end{array}$ & $\begin{array}{l}\text { Urban \& Indoor, } \\
4 \text { EDs, } 1 \mathrm{GW}\end{array}$ \\
\hline [129] & ADR+ & PDR, Energy & Simulator development & & OMNeT++ & \\
\hline$[130]$ & & PDR & Simulator extension & & ns-3 & \\
\hline [131] & & PDR & Simulator extension & & ns-3 & \\
\hline [132] & & Energy & Simulator extension & & ns-3 & D2D link \\
\hline [133] & Error model & PDR & Simulator development & & ns-3 & \\
\hline$[134]$ & & PDR, Energy & $\begin{array}{l}\text { Simulator development, } \\
\text { CSMA enhancement }\end{array}$ & & ns-3 & 4 EDs, $1 \mathrm{GW}$ \\
\hline [135] & $\begin{array}{c}\text { Heuristic parameter } \\
\text { selection }\end{array}$ & $\begin{array}{c}\text { Throughput, } \\
\text { System Lifetime }\end{array}$ & $\begin{array}{l}\text { Long-Lived LoRa } \\
\text { MAC protocol }\end{array}$ & & ns-3 & $\begin{array}{c}\text { Indoor, } \\
15 \text { EDs, } 1 \mathrm{GW}\end{array}$ \\
\hline [136] & & $\begin{array}{l}\text { Path Loss, PRR, } \\
\text { Energy, RSSI }\end{array}$ & & & & $\begin{array}{c}\text { Indoor, } \\
2-10 \mathrm{EDs}, 1 \mathrm{GW}\end{array}$ \\
\hline$[137]$ & & RSSI, SNR, PER & Architecture, Protocol stack & & & Urban \& Rural, 2 EDs, 3 GWs \\
\hline [138] & & Range, Energy, Packet loss & & & & Indoor, $1 \mathrm{ED}, 1 \mathrm{GW}$ \\
\hline [139] & $\begin{array}{c}\text { Edge computing, } \\
2 \text { ledgers } \\
\end{array}$ & $\begin{array}{l}\text { Throughput, CPU, } \\
\text { BW utilization }\end{array}$ & $\begin{array}{c}\text { Blockchain-based } \\
\text { architecture: HyperLoRa }\end{array}$ & & & $\begin{array}{c}\text { HyperLedger Fabric } \\
1 \mathrm{ED}(2000 \text { virtual }), 4 \mathrm{GWs}\end{array}$ \\
\hline$[140]$ & $\begin{array}{l}\text { Data storage } \\
\text { and retrieval }\end{array}$ & $\begin{array}{l}\text { Throughput, } \\
\text { Memory }\end{array}$ & $\begin{array}{c}\text { Blockchain-based } \\
\text { architecture }\end{array}$ & & & $\begin{array}{l}\text { Swarm storage, } \\
1 \mathrm{ED}, 1 \mathrm{GW}\end{array}$ \\
\hline [141] & $\begin{array}{c}\text { Management } \\
\text { architecture }\end{array}$ & Overhead & $\begin{array}{c}\text { Blockchain-based } \\
\text { architecture }\end{array}$ & & & $\begin{array}{l}1 \mathrm{ED}, 1 \mathrm{GW} \\
1 \text { Edge Node }\end{array}$ \\
\hline
\end{tabular}


graphical interface, where the topology of the network and nodes' measurements can be visualized. Key asset of COOJA is its high extension capability, which can provide insights in energy consumption and coverage (e.g., WSN-Maintain [149]), apart from the built-in results on network behavior and IoT-related protocols performance. Plugins that support ED mobility also exist [150]. Motes in COOJA are programmed either using $\mathrm{C}$ or Java.

\section{B. TOSSIM}

A similar approach to COOJA, is TinyOS Simulator (TOSSIM) [151], a discrete event simulator for EDs running TinyOS [152]. TOSSIM is also open source and its repository can be found in [153].

TinyOS can be found in devices that are operating under power and computing constraints in a concurrent way, while being easily modular. These 4 aspects are the main motivation points behind TinyOS development. TinyOS is open source itself and its main repository can be found in [154]. TinyOS, and as a result TOSSIM, are based on network embedded systems C (nesC) [155], [156], a limited dialect of C. Protocols that are of interest in the area of WSNs are supported, like Destination-Sequenced Distance-Vector Routing (DSDV) and Directed Diffusion.

As expected, TinyOS has been used extensively in IoT EDs, the behavior of which can be simulated via TOSSIM. In TOSSIM, the user can simulate the behavior of virtual EDs or even real motes (TOSSIM uses the same notation for EDs as COOJA) and the fact that deployed EDs run nesC permits the loading of code that runs in real deployment. Visualization is supported by TinyViz, with JTOSSIM being an alternative. TOSSIM is highly extensible with an example of this being the modeling of energy consumption by PowerTOSSIM [157]. Mobility support also exists [158] and numerical results about the performance of the network can be obtained. Regarding the coverage estimation of the network, there are no options for importing real map data in the simulator, to the best of our knowledge.

\section{CupCarbon}

A different approach to the previous simulators is CupCarbon [159], [160], [161]. The simulator focuses on simulating WSNs in the context of smart cities, supporting 3 of the most widely used technologies: ZigBee, LoRa and WiFi networking. CupCarbon can be found in [162].

CupCarbon as an environment comes with a number of advantages. Its Graphical User Interface (GUI) is one of its main benefits, providing visualizations with the use of multiple mapping methods, like Google Maps and Open Street Map. Simulator's documentation is fully explanatory [163] and includes all the mapping options, description of the GUI, presentation of the scripting language used in the simulator and a vast amount of examples that can be used in an effort to get familiar with the simulator environment or start examining WSNs and related algorithms, like Low-Energy Adaptive Clustering Hierarchy (LEACH) and Distributed Least Polarangle Connected Node (D-LPCN). The scripting language that is used is called SenScript and can be considered rather simple. It is based on a small number of commands for creating simple and more complex scenarios. Programming of EDs using Python is also supported in the latest version of the simulator.

Another important aspect of CupCarbon is the utility of event creation and integration in a simulation, which can be observed and reported by the nodes. Regarding the storage, extraction and visualization of the results, the user can rely on Comma Separated Values (CSV) files, log files in the form of text and the console of the simulator. Furthermore, graphs can be drawn inside the simulator, focusing on power consumption of the nodes.

\section{LoRaWANSim (Matlab)}

A recently proposed simulator called LoRaWANSim [127] has been implemented in Matlab. Due to name conflict with a simulator discussed in Subsection $F$, we will refer to the current simulator as LoRaWANSim (Matlab). Simulator's code is accessible via [164].

LoRaWANSim (Matlab), as its name suggests, focuses solely on LoRa/LoRaWAN systems. It can simulate both LoRa PHY layer and LoRaWAN MAC layer while considering EU868 limitations. The simulator offers many advantages in terms of accurate result extraction, most notably the support of numerous GWs and the account of interference of all types (among EDs, among GWs and a mixture of these types). Since the simulator is implemented in Matlab, this is the programming language used to setup the simulations.

There is no GUI implemented, but a mapping is drawn with no real terrain, serving mainly as a visualization of EDs and GWs position relative to each other. Results include performance of the network in terms of PDR and energy consumption and are accurate when compared to analytical models that are discussed in [127]. Being a simulator focused on LoRaWAN, it supports the key algorithmic function of the protocol: ADR performed by NS. Mobility is not supported.

\section{E. LoRaSim (Java)}

Another simulator dedicated to studying LoRaWAN systems is presented in [128], developed using the Java programming language. In Subsection $F$ a simulator with the same name is described, so we refer to the current simulator as LoRaSim (Java).

LoRaSim (Java) is a discrete event simulator. It has been developed to simulate devices operating in US915 band. Configuration files for GW, EDs and the network can be used as input to the simulator, where the typical parameters of TP, SF, CR and BW are among those that can be described in these files. Results about network performance can be obtained, such as PDR. Algorithmic issues can be examined as the one described in [114], where a proposed carrier sensing mechanism is compared to pure ALOHA in terms of power consumption and system capacity, using the developed simulator.

No GUI details are provided, however LoRaSim (Java) supports the placement of all LoRa systems nodes ( GW and 
EDs) in a coordinated system, but not using a real map. EDs' mobility is not examined in the corresponding references.

\section{F. LoRaSim / MoTSim / LoRaWANSim / LoRaEnergySim / LoRaMACSim (Python)}

LoRaSim is a discrete event simulation environment for LoRa networks. The simulator has been developed using the Python programming language and the simulation framework SimPy and thus it should not be confused with the namesake simulator discussed earlier. Its main target is to examine the collisions in LoRa systems and provide insights on the topic of scaling such networks.

Relevant published works for LoRaSim are [55], [121]. In these references, the capabilities of the simulator are discussed, while the various simulations along with their setups are presented. The simulator can support the creation of multiple LoRa EDs and their communication with one or more GWs. LoRa EDs are simulating the behavior of an element that is based on SX1272 chipset, while LoRaSim's GWs are based on SX1301. The adjustable parameters in LoRaSim are: TP, CF, SF, BW and CR. Results can be obtained about the network performance, like DER. A map with the relative positions of GWs and EDs can be drawn, however it it not based on real environment. There is no further visualization and mobility is not supported. Simulator can be found in [165].

LoRaSim proved to be a big success and several research teams work on the simulator, in an effort to modify or extend the environment to suit their needs. One such research contribution can be found in [63], where the authors base their MoTSim simulator in LoRaSim, trying to examine their proposed MAC layer protocol. Subsequently, another research team has extended the LoRaSim simulator via implementing the necessary support for LoRaWAN protocol [122]. The extended version of the simulator is called LoRaWANSim (not to be confused with the previously discussed simulator). Extensions are focused on MAC layer with the support of DL data and ACKs, duty cycle limitations, collision models, retransmissions and data rate adaptation based on unsuccessful packet transmissions. Results include energy consumption and network performance indicators.

A further improvement to the original simulator came from a third research team and is described in [123], named LoRaEnergySim. Simulations are organized in a framework that contains three main entities: ED, air interface and GW. Important aspect of air interface class is the consideration of known propagation models, like log-distance model and the COST-231 model, which fits nicely the European deployments. For ED, a realistic energy model is considered based on LoRaWAN_EFM32, whereas the GW is based on an iC880A module. Algorithmic-wise, the ADR algorithm as implemented by TTN is also included. Simulator is available in [166].

Based on LoRaEnergySim, another research contribution is described in [65], with additional capabilities for the simulator. The LoRaMACSim takes into account two more well-known propagation models; the Rayleigh fading and the ETSI TR 136 942. Moreover, the topic of multiple access can be studied thoroughly due to the consideration of the slotted ALOHA and CSMA/CA protocols, apart from the usual approach of pure ALOHA. LoRaMACSim is also available, in [124].

\section{G. LoRa-MAB}

One more discrete event simulator built with the Python programming language and the SimPy framework is LoRaMulti-Armed Bandit (LoRa-MAB) [125]. The goal of the simulator is to provide an environment for estimating resource allocation in LoRa networks in an intelligent distributed way and analyze the scalability potential of a LoRa network.

To this end, the authors have developed a simulator that has multiple input parameters, supporting a plethora of EDs and GWs, while assuming real radio propagation models and other realistic impact factors like capture effect. EDs are distinguished to normal EDs and smart EDs, with the latter being able to perform the proposed intelligent distributed algorithm for resource allocation. Results can be drawn for energy and other performance metrics, like packet reception rate which can be thought as PDR, whereas coverage can be examined but not with the use of a real map.

EDs' mobility is not discussed and a GUI is not provided. LoRa-MAB is available in [167].

\section{H. LoRaFREE}

The final discrete event simulator built with the Python programming language and the SimPy framework to be discussed in this paper is LoRa Fine-Grained Scheduling for Reliable and Energy-Efficient Data Collection in LoRaWAN (LoRaFREE) [126]. This simulator targets the validation of the namesake, FREE algorithm.

The authors in [126] have developed a highly configurable simulator with several input parameters, including the usual SF, BW, CR and TP, which can support multiple EDs. LoRaFREE uses realistic assumptions in a number of areas, like a packet error model, the imperfect orthogonality of spreading factors, fading events and duty cycle limitation for EDs and GWs (European deployments). Results can be drawn for numerous performance metrics, with the most important of them being the DDR, collisions and several ACK-related metrics. Energy consumption can be measured thoroughly in the simulator environment. EDs and GWs can be drawn in a non-real map. Algorithmic issues can be examined, as depicted by the study of the proposed mechanism.

No additional GUI is provided apart from the projection of EDs and GWs in a coordinated system. Mobility is not taken into consideration. LoRaFREE can be found in [168].

\section{I. $O M N e T++$}

The Objective Modular Network Testbed in C++ (OM$\mathrm{NeT++}$ is a simulation environment that is based on modules written in $\mathrm{C}++$. With these modules, simulations for various networking systems, both wireless and wired, may be created. It follows the discrete event simulation pattern, as many other simulators on the field.

Its popularity among other simulation environments is justified by the numerous available frameworks. The INET 
framework, which is the most widely used, includes models for examining several known protocols from all the layers of the known TCP/IP protocol suite, for nodes that can be both at fixed position or in motion. Other commonly used frameworks are SimuLTE, which is based on INET and is used for evaluating the behavior of LTE and LTE-A networks, as its name suggests, Simu5G, which extends the simulation capabilities to $5 \mathrm{G}$ networks, the Artery framework that targets Vehicle to Everything (V2X) simulations, Castalia framework [169], which targets the simulation of networks consisted of energy-constrained devices like WSNs, and PAWiS simulation framework [170], which also strives for WSN simulations and others.

With the increasing adoption of LoRa networks, it was expected that a framework for such systems would be developed. The Framework for LoRa (FLoRa) [129] simulates the behavior of EDs, GWs and NS in a LoRa network. The adjustment of parameters is an option and a dynamic scheme of parameter modification reminiscent to ADR is supported. Details about the framework can be found in the corresponding web page [171] and its repository [172], since FLoRa follows the open source approach. The capabilities of gathering statistics regarding the transmissions (PDR) and the power consumption are of great importance for its usage. FLoRa supports an energy model based on power consumption of SX1272/73 chipset.

It is worth to mention that OMNeT++ (and as a result FLoRa) provides an intuitive GUI that offers visualization of the network and even simulations based on realistic physical environment, whereas tools to log events and analyze them also exist.

\section{J. $n s-3$}

Final simulator to be discussed, with extensive use in networking research, is network simulator 3 (ns-3) [173]. It is part of the simulator series ns, with its core being based on $\mathrm{C}++$ programming language, supporting also the development of simulations using Python. ns-3 utilizes built-in libraries, which can be combined for developing various networking systems, IP-based or not.

Simulations in ns-3 are performed with the use of 5 main Abstract Data Types (ADTs): the node, the application, the channel, the network device (netdevice) and the topology helper. More information can be found in the official documentation [174]. The vast amount of libraries assists the development and simulation of different kinds of networks; from 3GPP networks to IEEE 802.11 systems and from networks that are based on modern paradigms like SDN to systems that experience intense heterogeneity with new and old technologies coexisting. In this context, LPWANs can be considered and more specifically LoRaWAN systems, which are subject of intense research.

As a result, a module able to simulate LoRaWAN networks is available and can be found in [175], while published works [57], [130] and [131] explore and greatly extend the LoRaWAN capabilities of ns-3. In the same frame, work published in [132] extends these modules for realistic energy consumption estimation. Other efforts on ns-3 LoRaWAN modules development also exist, with [133] focusing on PDR using newly developed applications in ns-3 implemented by another research group, whereas another research team investigates the feasibility of CSMA protocol on top of LoRa in [134]. Moreover, several recording commands and log file creation commands can aid the analysis of network's behavior, whereas the input of a simulation is highly configurable. Simulations to $\log$ any interested network performance metric can be developed. Algorithmic processes are regularly simulated in ns-3 and in the context of LoRaWAN module such efforts do exist, ranging from the common ADR algorithm to typical WSN-related algorithms, like Pruning algorithm [175].

Considering the graphical interface, NS-3 is a terminalbased simulator. However, visualization can be achieved with the use of an animator like NetAnim or PyViz. ns-3 provides several mobility models and coverage can be examined, while the use of reminiscent to real environments further improves the validity of the extracted results. ns- 3 is an open source project [176].

\section{K. Summary of Simulation Environments}

A comprehensive summary of the presented simulation environments is given in TABLEIX. The simulators are examined in terms of programming languages supported, the existence or not of a GUI and the capabilities of them in terms of EDs' energy consumption, network coverage, network performance (throughput, data rate, PDR or related metrics), ED mobility and the support of simulating algorithmic processes or protocols. A performance factor that could have also been discussed is scalability, i.e., the number of EDs that can be simulated, however there is no apparent distinction in this area; all of these simulators are able to provide results in simulations crowded with EDs.

\section{An Overview of Cloud Platforms For the SERVER SIDE OF LPWANS}

From user's perspective, most of operations in IoT systems and consequently in LPWAN systems, follow the cloud paradigm. In recent years, the Everything-as-a-Service (XaaS) approach has established itself as a growing field in the area of cloud computing. Cloud computing refers to the offering of virtualized computing resources, with several cloud models in exist. The three main representatives, according to NIST, are Software-as-a-Service (SaaS), PaaS and Infrastructure-asa-Service (IaaS). NIST definitions for all three of them are inclusive [177], however we provide our own definitions here:

- SaaS: The ability of an end user to use existing applications developed by the cloud service provider, via an Application Programming Interface (API) or a client request. End user might be granted authorization to configure a few parameters of the application, but neither user-developed applications are allowed nor access to the underlying platform or hardware infrastructure is permitted.

- PaaS: The ability of an end user to deploy its own software, assuming software's technology can be supported by the cloud environment (e.g., Java applications 
TABLE IX

OVERVIEW OF SIMULATION ENVIRONMENTS WITH A LORA/LORAWAN FOCUS

\begin{tabular}{|c|c|c|c|c|c|c|c|c|c|}
\hline $\begin{array}{c}\text { Simulation } \\
\text { Environment }\end{array}$ & Ref. & Target & Programming & GUI & Energy & $\begin{array}{l}\text { Coverage } \\
\text { (Map) }\end{array}$ & $\begin{array}{c}\text { Network } \\
\text { Performance }\end{array}$ & $\begin{array}{c}\text { ED } \\
\text { Mobility }\end{array}$ & $\begin{array}{l}\text { Algorithmic } \\
\text { Processes }\end{array}$ \\
\hline COOJA & $\begin{array}{l}{[143]} \\
{[144]}\end{array}$ & $\begin{array}{l}\text { IoT generic } \\
\text { (Contiki EDs) }\end{array}$ & C, Java & Built-in & $\checkmark$ & $\begin{array}{c}\checkmark \\
\text { (real) }\end{array}$ & $\checkmark$ & $\checkmark$ & $\checkmark$ \\
\hline TOSSIM & [151] & $\begin{array}{c}\text { IoT generic } \\
\text { (TinyOS EDs) }\end{array}$ & nesC, $\mathrm{C}, \mathrm{C}++$ & $\begin{array}{c}\text { TinyViz, } \\
\text { JTOSSIM }\end{array}$ & $\checkmark$ & - & $\checkmark$ & $\checkmark$ & $\checkmark$ \\
\hline CupCarbon & $\begin{array}{l}{[159]} \\
{[160]} \\
{[161]}\end{array}$ & $\begin{array}{l}\text { EDs with Zigbee, } \\
\text { WiFi, LoRa radio }\end{array}$ & $\begin{array}{l}\text { SenScript, } \\
\text { Python }\end{array}$ & Built-in & $\checkmark$ & $\begin{array}{c}\checkmark \\
\text { (real) }\end{array}$ & $\checkmark$ & $\checkmark$ & $\checkmark$ \\
\hline LoRaWANSim & [127] & $\begin{array}{l}\text { LoRa/LoRaWAN } \\
\text { specific }\end{array}$ & Matlab & - & $\checkmark$ & $\begin{array}{c}\checkmark \\
\text { (not real) }\end{array}$ & $\checkmark$ & - & $\checkmark$ \\
\hline LoRaSim & $\begin{array}{l}{[114]} \\
{[128]}\end{array}$ & $\begin{array}{c}\text { LoRa/LoRaWAN } \\
\text { specific }\end{array}$ & Java & - & $\checkmark$ & $\begin{array}{c}\checkmark \\
\text { (not real) }\end{array}$ & $\checkmark$ & - & $\checkmark$ \\
\hline $\begin{array}{l}\text { LoRaSim, } \\
\text { LoRaWANSim, } \\
\text { MoTSim, } \\
\text { LoRaEnergySim, } \\
\text { LoRaMACSim }\end{array}$ & $\begin{array}{l}{[55]} \\
{[63]} \\
{[65]} \\
{[121]} \\
{[122]} \\
{[123]} \\
\end{array}$ & $\begin{array}{c}\text { LoRa/LoRaWAN } \\
\text { specific }\end{array}$ & Python & - & $\checkmark$ & $\begin{array}{c}\checkmark \\
\text { (not real) }\end{array}$ & $\checkmark$ & - & $\checkmark$ \\
\hline LoRa-MAB & [125] & $\begin{array}{l}\text { LoRa/LoRaWAN } \\
\text { specific }\end{array}$ & Python & - & $\checkmark$ & $\begin{array}{c}\checkmark \\
\text { (not real) }\end{array}$ & $\checkmark$ & - & $\checkmark$ \\
\hline LoRaFREE & [126] & $\begin{array}{c}\text { LoRa/LoRaWAN } \\
\text { specific }\end{array}$ & Python & - & $\checkmark$ & $\begin{array}{c}\checkmark \\
\text { (not real) }\end{array}$ & $\checkmark$ & - & $\checkmark$ \\
\hline $\begin{array}{l}\text { OMNeT++ } \\
\text { (FLoRa) }\end{array}$ & [129] & $\begin{array}{c}\text { Network generic } \\
\text { (LoRa/LoRaWAN } \\
\text { specific) }\end{array}$ & $\mathrm{C}++$ & Built-in & $\checkmark$ & $\begin{array}{l}\checkmark \\
\text { (real) }\end{array}$ & $\checkmark$ & $\checkmark$ & $\checkmark$ \\
\hline NS-3 & $\begin{array}{c}{[57]} \\
{[130]} \\
{[131]} \\
{[132]} \\
{[133]} \\
{[134]} \\
{[175]}\end{array}$ & $\begin{array}{c}\text { Network generic } \\
\text { (LoRa/LoRaWAN } \\
\text { specific) }\end{array}$ & $\mathrm{C}++$, Python & $\begin{array}{l}\text { NetAnim, } \\
\text { PyViz }\end{array}$ & $\checkmark$ & $\begin{array}{c}\checkmark \\
\text { (partly real) }\end{array}$ & $\checkmark$ & $\checkmark$ & $\checkmark$ \\
\hline
\end{tabular}

will require a certain environment to run in the cloud infrastructure). End user might be granted authorization to configure a few parameters of the environment, but has no control over the underlying platform or the hardware infrastructure.

- IaaS: The ability of an end user to deploy entire platforms in the cloud environment, assuming the platform's components can be supported by the cloud infrastructure (e.g., a system component requiring a minimum amount of memory to run). End user is given the option to configure the platform as needed, e.g., OS, storage, but has no control over the underlying hardware infrastructure.

More on the concept of cloud computing can be found in [178], in which the Database-as-a-Service (DBaaS) model -an emerging service model in the area of cloud support to IoT systems- is presented. An informative resource is also [87], where the topic of IoT forensics (Forensics-asa-Service (FaaS)) is discussed, giving valuable insights in security aspects of IoT networking based on cloud computing.

Such a cloud service paradigm has already been discussed. RPMA is characterized as a PaaS LPWAN solution, meaning that Ingenu offers the required tools to end users for developing their own applications on top of RPMA platform. Apart from RPMA, a number of companies offer SaaS and IaaS solutions in the LPWAN domain, by allowing access to backend servers, storage space or deployed infrastructure.

Numerous key industry entities operating in this area could be mentioned [179]. Amazon's Amazon Web Services (AWS) is a well-established platform offering cloud solutions and in the context of IoT, IoT Core has great interoperability with LoRaWAN systems. Microsoft provides similar capabilities with its Azure IoT platform. Google, also invests heavily in such a platform with Google Cloud Platform (GCP). Alibaba shows substantial momentum in this field with its Alibaba Cloud and the corresponding IoT platform. IBM serves IoT Cloud needs with its IBM Watson IoT Platform. Tencent runs the namesake cloud platform and offers solutions via its IoT Hub. Finally, Oracle is another option to be considered in this area, with Oracle Cloud Infrastructure (OCI) and IoT Intelligent Applications Cloud.

\section{Data Management in LPWANs}

LPWAN systems are part of the $3^{\text {rd }}$ IoT generation [23], which focuses on cloud storage and processing, by following data-centric management [24] and data-driven networking [180]. The entirety of data-driven networking in the context of LPWANs faces the key data challenges of the five "V"s of big data -as they are mentioned in the literature- namely: data generated in high Volumes, with extreme Velocity, having intense Variety, being Valuable and needed to check their Veracity. To address those challenges, LPWAN architectures have already emerged [181], [182]. For the definition of such architectures common reference models [183] from the broader Industry 4.0 sector are used, namely: the Reference Architectural Model Industrie 4.0 (RAMI4.0) [184], the Industrial Internet Reference Architecture (IIRA) [185], the International Data Spaces 
Reference Architecture Model (IDS-RAM) [186] and the IoT World Forum's (IoTWF) Reference Model [187]. While for the lower layers of such models (covering the communication and connectivity aspects) any of the LPWAN technologies provided in previous sections can be used, in this section the focus is on operations upon data.

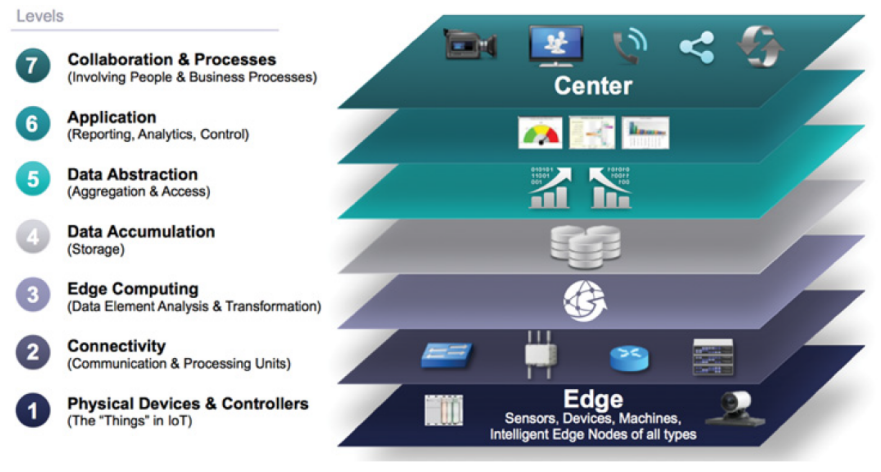

Fig. 20. IoTWF Reference Model [187]

\section{A. Fundamental data processes for LPWANs}

Data management in LPWANs includes all the fundamental processes that are applied on data when it comes to communications systems; namely: data acquisition, data storage, data visualisation, and data analysis.

Data acquisition is at the heart of sensing and actuating systems, since EDs' main operation is collecting and/or acting on data. Two distinct approaches exist: the pull model and the push model. The pull model expects from the user (usually sitting beyond the server-side part of the system) to ask for the data. Key representative of pull model is HyperText Transfer Protocol (HTTP), where a user can apply the GET method to retrieve information. On the other hand, the push model supports the dispatch of data to the interested user by the time the data are transmitted by EDs. Message Queuing Telemetry Transport (MQTT) protocol and Webhooks are well-established and widely used push methods. Due to the increasing interest in WSN, Organization for the Advancement of Structured Information Standards (OASIS), which develops MQTT, is working towards the standardization of MQTT for Sensor Networks (MQTT-SN).

Data storage is also of vital importance for the successful setup of an LPWAN system. An initial approach is to store data in plain files of .txt (less frequently used), .csv or .json extension. JavaScript Object Notation (JSON) has gained a lot of attention in recent years and along with CSV are considered the main human-readable data representation today. eXtensible Markup Language (XML) is another option and its applicability in semantic web and Web of Things (WoT) [188] context can be proven useful to system developers focusing on such data inter-exchange. Efficient XML Interexchange (EXI) is another step towards this direction [189], having been developed for computationally constrained devices. However, a more sophisticated approach would dictate the utilization of a database paradigm. Several options exist, with the main considerations being: a document-oriented database (e.g., Dynamo DB) that may also export data in the form of popular representations like .csv and .json extensions files, a timeseries database optimized for the flow of IoT data (e.g., Influx DB), or a graph-based database where each node could be a UL transmission distinguished by the reception time from the $\mathrm{GW}$, with the sequence of transmissions creating a chain (e.g., Dgraph, Neo4j).

Data visualisation and analysis is applied in the stored data, in order to transform them to information. Grafana is an open platform that is utilized in numerous established projects. Mathworks has developed a solution that not only offers visualization capabilities but also supports other operations upon data: the ThingSpeak platform. In the same field, platforms like TagoIO are also widely used. Matlab is used in ThingSpeak platform for data analysis and processing, whereas TagoIO supports Javascript (Node.js) and Python. Naturally, LPWANs support the current evolution in IoT data analysis (and especially in industrial data management), towards exploiting Artificial Intelligent (AI) toolkit. In this context, ML models can be utilized, based on well-established toolboxes or frameworks like PyTorch and Tensorflow. ML models can be deployed not only in system components with high computing capabilities, like a GW, an NS or a monitoring platform, but also in EDs themselves, thanks to TinyML [190].

\section{B. Advanced data-driven services on top of LPWANs}

Beyond the fundamental data processes, innovative datadriven concepts have emerged (especially in the industrial sector). Some of the most critical ones are: i) common frameworks for representation of the nodes of a network, referring mainly to the concept of Asset Administration Shell (AAS) [191], including common data formats and communication protocols, referring mainly to the work conducted by Open Platform Communications (OPC) Foundation and especially the OPC-Unified Architecture (OPC-UA) group, and ii) the potential of exploiting the common representation and data modeling schemes towards the digital twinning of LPWAN systems.

Representation and Data modeling. The term "assets" (as defined within the manufacturing sector) refers to any interconnected device, sensor, actuator, machine, or even processes and systems, inside a controlled communication environment (e.g., inside a manufacturing plant). Considering this, AAS is the basis on top of which discovery, access and description of an asset are made [102]. The specifications of the AAS are defined in IEC 63278 [192], while its implementation is a subject of many open source approaches such as the AASX [193] and the Eclipse Basyx [194]. Discovery is a fundamental mechanism in AAS, and a prerequisite for any communication establishment. There are different approaches for that, including the use of directories-style systems, search engines, crawlers, networking protocols, etc. Access refers to a set of APIs used for accessing the assets. The use of APIs is selected in an effort to ensure unified access, at the cost of requesting every resource to implement them. Asset description refers to data models for describing the asset 
procedures and status, as for instance the OPC-UA models, defined by the OPC Foundation. The communication model of OPC-UA fits well to the LPWAN networks since it is a pub/sub protocol as described in open62541 [195].

One step beyond the default data models of OPC-UA, new information models are created which are called Companion Specifications and they are specific to vertical industries (as for instance those defined by the Mechanical Engineering Industry Association VDMA). In addition, the Digital Twin (DT) community has built related tools and models like the DT Definition Language (DTDL) [196] that specifies an Industry 4.0 language used for inter-AAS communication.

The digital twin concept [102]-[105]. DT refers to the digital presence of any "asset" enriched with full administration capabilities. It is based on the concept of emulation, but is fed with real data and is able to affect the real processes among the "assets". With its ability to interconnect a physical entity with a digital one and allowing the flow of data between them, the DT concept is expected to play a major role in the realization of IIoT [106], [107] and Industry 4.0. AAS, as presented above, is the key enabler of the DT provisioning. The realization of a DT could be based on emulation-oriented systems, such as that provided by the Emulated IoT (ELIoT) [197], [198]. In ELIoT, EDs can be abstracted as clients and NS as server and an LPWAN system can be emulated as a client-server model. However, already dedicated cloud-based tools on digital twinning have emerged, as for instance the PaaS solution of Azure DT [199]. For the communication part of a system, and its data flows, the NodeRed tool is also considered as a DT-enabler.

\section{CONCLUSION}

LPWAN systems is an emerging connectivity solution looking to embrace the challenge of satisfying the needs of the $3^{\text {rd }}$ generation of IoT networking. Their main target is to serve connectivity demands in terms of long range, low power and low data rates, with low setup and operational cost. LPWAN-related technologies have already been established in several (3GPP and non-3GPP) standards, covering various application demands and network performance requirements. As revealed from the technology comparison study presented in this paper, a standout solution for LPWANs in unlicensed bands is the LoRa/LoRaWAN networking approach, referring to systems that use LoRa PHY and LoRaWAN MAC technologies. Overviews of both technologies are provided, in an effort to serve as reference points to fundamental processes and performance capabilities of LoRa/LoRaWANenabled LPWANs.

In this framework, the research area of LPWANs is flooded with a plethora of approaches and studies, which are classified to analytical approaches, simulation studies and real implementations/measurement campaigns. Based on the stateof-the-art study and assessment in this paper, the majority of the research approaches in the area is built around the choice/adaptation of the re-configurable parameters at PHY and MAC layers. In addition, LPWANs have been proven to be good candidates for applied research, targeting real measurements and setups. This is mainly due to the low establishment cost and the rich open source solutions available. In the case of field implementations, LPWAN systems tend to be supported by cloud infrastructures; an observation that confirms the current trend on virtualizing as much as possible of the LPWANs' functionality. However, due to the massiveness characteristic (a key aspect of IoT EDs), large scale research studies still need to be conducted via simulations. Considering this, a point-to-point comparison of the available simulators for LPWANs has been provided, revealing the pros and cons of the (already long list of) available tools.

Finally, it is apparent that the LPWANs are not only about connectivity; they are actually one of the key features of the broader evolution towards a data-driven IoT world. As such, they can be considered potential candidates for industrial solutions, where advanced data-driven services are designed based on recent advancements, like the AAS and the Digital Twinning.

\section{APPENDIX A \\ A QuAntitative STUdy on LoRa Modulation}

LoRa is an implementation of $\mathrm{CSS}^{8}$, which is a member of Spread Spectrum (SS) family [200]. SS as a technique strives to spread the signal over a wider BW, achieving better performance in terms of jamming resistance, interference, unintended signal detection and eavesdropping [201].

The spreading process is based in two main elements: coding and pseudorandomness. The block diagram can be seen in Fig. 21.

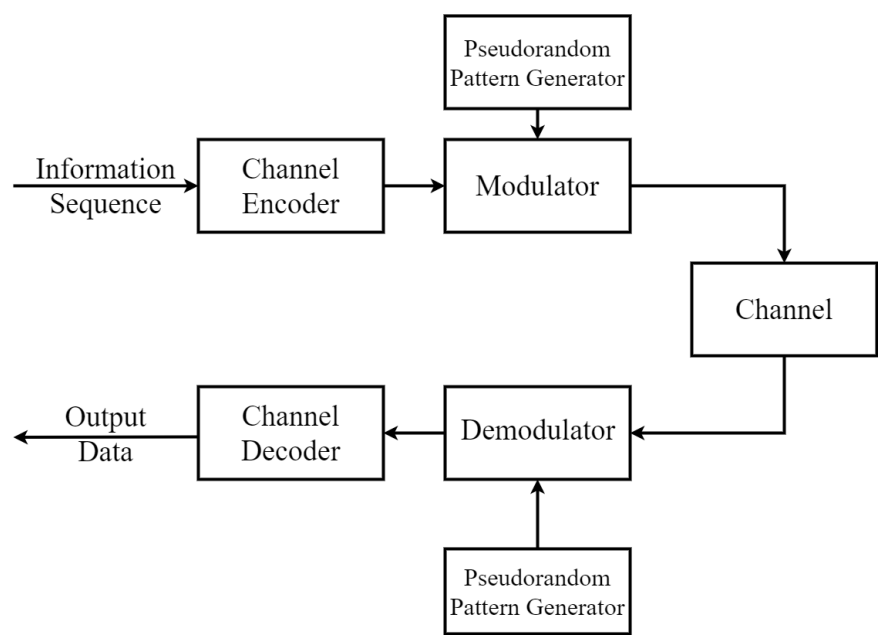

Fig. 21. Spread Spectrum block diagram [201]

Several SS approaches have been proposed over the years, with Direct Sequence SS (DSSS) and Frequency Hopping SS (FHSS) being the main representatives. Other methods include Time Hopping SS (THSS) and Hybrid SS (HSS). LoRa CSS implementation is considered a DSSS alternative [73].

\footnotetext{
${ }^{8}$ LoRa networks are not the only WSN approach that is relying on CSS for its PHY layer. IEEE 802.15.4a is another example of CSS utilization and can be used for Zigbee connectivity.
} 
The amount of BW spread is denoted by SF. Assuming an input $\mathrm{BW} R$, one can spread the signal transmission over a BW $W$. Then $W$ is equal to $R$ times the SF, so:

$$
W=S F * R \Leftrightarrow S F=\frac{W}{R}
$$

For an $S F=3$, it means that the signal is transmitted over a BW that is 3 times wider that the input BW, as depicted in Fig.22. It is worth to mention that if we calculate the integrals of the two areas in Fig.22, depicting the initial input and the spread one, they should be equal.

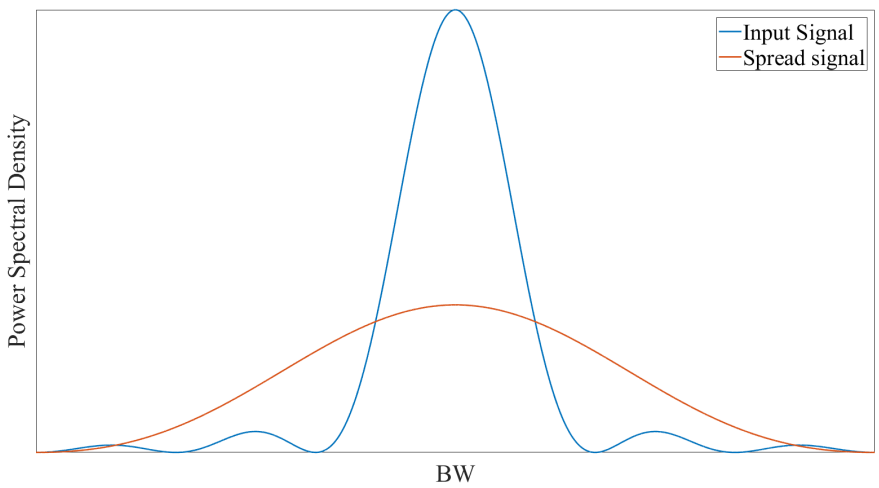

Fig. 22. Power Spectral Density with respect to BW for an input signal and a spread signal

We proceed by defining the term of chip, which is the way to encode information in SS systems. Practically, the spreading factor is a chip sequence, so it indicates the number of bits used for a symbol. If we define that the symbol is a chirp in CSS modulation, then for a known $S F$ the ways to encode information in a symbol are $2^{S F}$.

The next step is to define the terms of rate and duration for chip and symbol, and eventually bit rate. The chip rate will be equal to the spread $\mathrm{BW} W$, so:

$$
R_{c}=W
$$

The symbol rate will be equal to the chip rate divided by the number of ways to encode information in one symbol, so:

$$
R_{S}=\frac{R_{c}}{2^{S F}}
$$

In this context, the reciprocal of rate is duration, so we can also define $T_{c}$ and $T_{s}$ as ${ }^{9}$ :

$$
\begin{gathered}
T_{c}=\frac{1}{R_{c}}=\frac{1}{W} \\
T_{S}=\frac{1}{R_{S}}=\frac{2^{S F}}{W}
\end{gathered}
$$

Finally, the bit rate will be equal to the number of bits used for a symbol divided by the duration of a symbol (or equivalently multiplied with the rate of a symbol):

$$
R_{b}=\frac{S F}{T_{s}}
$$

\footnotetext{
${ }^{9}$ If we define the bit duration in the same way, we get the fraction $\frac{W}{R}=$ $\frac{T_{b}}{T_{C}}=S F$. This quantity can also be found in literature as processing gain.
}

However, the bit rate in LoRa is slightly lower than that, due to the inclusion of a FEC scheme. LoRa defines a 4-value Rate Code, picking an element from the set $\left\{\frac{4}{5}, \frac{4}{6}, \frac{4}{7}, \frac{4}{8}\right\}$. Rate code is a fraction that has as nominator the amount of useful information carried to a message and as a denominator the total number of bits, i.e., information bits plus redundant bits for error correction.

$$
\text { Rate Code }=\frac{4}{4+C R}
$$

Ultimately, the bit rate in LoRa considering FEC and equations A5, A6, A7 will be the one given in the corresponding LoRa section (Section VII, equation 1):

$$
R_{b}=S F * \frac{4}{4+C R} * \frac{W}{2^{S F}}
$$

In CSS modulation, information is carried using the notion of chirp signal. A chirp is a signal that its frequency is changing over time, either moving from lower frequencies to higher (up-chirp) or the opposite direction (down-chirp). The waveforms of an up-chirp and a down-chirp can be seen in Fig.23.
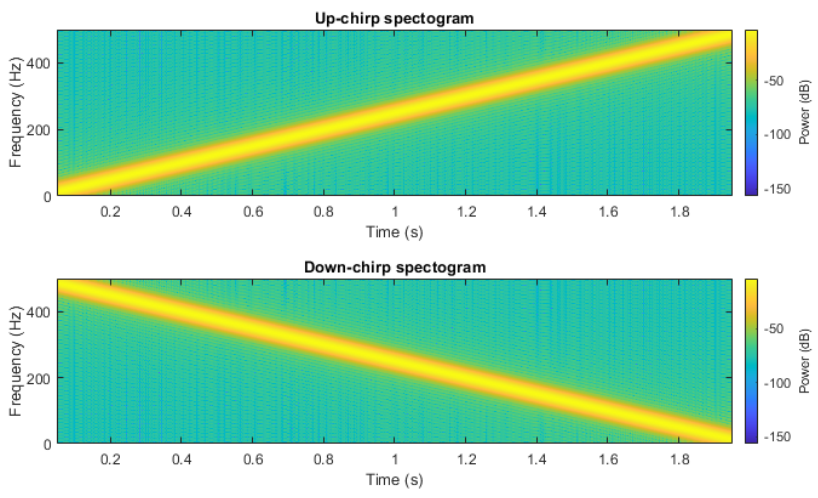

Fig. 23. Spectogram for an up-chirp (up) and a down-chirp (down)

LoRa uses a scheme of cyclic shifting when reaching the upper bound in the case of up-chirp (or lower bound in the case of down-chirp).

\section{ACKNOWLEDGMENT}

This work has been co-funded by Greek national funds through the Operational Program Competitiveness, Entrepreneurship, and Innovation, under the call RESEARCH - CREATE - INNOVATE (project CityZEN, code: T1EDK 02121).

\section{ACRONYMS}

TABLE $X$

ACRONYMS TABLE

\begin{tabular}{ll}
\hline Acronym & Definition \\
\hline \hline 16-QAM & 16-Quadrature Amplitude Modulation \\
3GPP & 3rd Generation Partnership Project \\
8PSK & 8-Phase Shift Keying \\
AAS & Asset Administration Shell \\
ABP & Activation By Personalization
\end{tabular}


TABLE X

ACRONYMS TABLE (CONT'D)

\begin{tabular}{|c|c|}
\hline ACK & Acknowledgment \\
\hline ADR & Adaptive Data Rate \\
\hline ADT & Abstract Data Type \\
\hline AES & Advanced Encryption Standard \\
\hline AI & Artificial Intelligent \\
\hline API & Application Programming Interface \\
\hline APP & Application layer \\
\hline AWGN & Additive White Gaussian Noise \\
\hline AWS & Amazon Web Services \\
\hline BER & Bit Error Rate \\
\hline BLE & Bluetooth Low Energy \\
\hline BPSK & Binary Phase Shift Keying \\
\hline BS & Base Station \\
\hline BT & Bandwidth-symbol Time \\
\hline BW & Bandwidth \\
\hline C-DRX & Connected mode DRX \\
\hline CAD & Channel Activity Detection \\
\hline CDMA & Code Division Multiple Access \\
\hline $\mathrm{CF}$ & Carrier Frequency \\
\hline $\mathrm{CH}$ & Cluster Head \\
\hline CIoT & Cellular IoT \\
\hline CMAC & Cipher-based Message Authentication Code \\
\hline $\mathrm{CR}$ & Coding Rate \\
\hline $\mathrm{CRC}$ & Cyclic Redundancy Code \\
\hline CSMA & Carrier Sense Multiple Access \\
\hline CSMA/CA & Carrier Sense Multiple Access / Collision Avoidance \\
\hline CSS & Chirp Spread Spectrum \\
\hline CSV & Comma Separated Values \\
\hline CTR & Counter (AES mode) \\
\hline CapEx & Capital Expenditure \\
\hline COAP & Constraint Application Protocol \\
\hline D-LPCN & Distributed Least Polar-angle Connected Node \\
\hline DBaaS & Database-as-a-Service \\
\hline DD-UNB & Dynamic Downlink Ultra Narrow Band \\
\hline DDR & Data Delivery Ratio \\
\hline DER & Data Extraction Rate \\
\hline DFRL & Deep Federated Reinforcement Learning \\
\hline DFT & Discrete Fourier Transform \\
\hline DL & Downlink \\
\hline DSDV & Destination-Sequenced Distance-Vector Routing \\
\hline DSL & Digital Subscriber Line \\
\hline DSSS & Direct Sequence Spread Spectrum \\
\hline DT & Digital Twin \\
\hline DTDL & DT Description Language \\
\hline EC-GSM-IoT & Extended Coverage-GSM-IoT \\
\hline ED & End Device \\
\hline EDGE & Enhanced Data rates for Global Evolution \\
\hline ELIoT & Emulated IoT \\
\hline ETSI & European Telecommunications Standards Institute \\
\hline EXI & Efficient XML Interexchange \\
\hline EXP3 & Exponential-weight for Exploration and Exploitation \\
\hline FDMA & Frequency Division Multiple Access \\
\hline FEC & Forward Error Correction \\
\hline FER & Frame Error Rate \\
\hline FFT & Fast Fourier Transform \\
\hline $\mathrm{FH}$ & Frequency Hopping \\
\hline FSK & Frequency Shift Keying \\
\hline FUOTA & Firmware Update Over The Air \\
\hline FaaS & Forensics-as-a-Service \\
\hline GCP & Google Cloud Platform \\
\hline GERAN & GSM EDGE Radio Access Network \\
\hline GFSK & Gaussian Frequency Shift Keying \\
\hline GMSK & Gaussian Minimum Shift Keying \\
\hline GPRS & General Packet Radio Service \\
\hline GUI & Graphical User Interface \\
\hline GW & Gateway \\
\hline HARQ & Hybrid Automatic Repeat Request \\
\hline HTTP & HyperText Transfer Protocol \\
\hline HVAC & Heating, Ventilation and Air Conditioning \\
\hline IDS-RAM & International Data Spaces Reference Architecture Mo \\
\hline IIRA & Industrial Internet Reference Architecture \\
\hline IIoT & Industrial IoT \\
\hline IMT & International Mobile Telecommunications \\
\hline
\end{tabular}

TABLE $X$

ACRONYMS TABLE (CONT'D)

IP

ISM Industrial, Scientific, Medical

IT Information Technology

IaaS Infrastructure-as-a-Service

IoT Internet of Things

IoTWF IoT World Forum

JSON JavaScript Object Notation

KPI Key Performance Indicator

LBT Listen Before Talk

LCS Lightweight Carrier Sensing

LEACH Low-Energy Adaptive Clustering Hierarchy

LOS Line of Sight

LPWAN Low-Power Wide Area Network

LR-FHSS Long Range - Frequency Hopping Spread Spectrum

LTE-M Long Term Evolution-Machine type communication

LTN Low Throughput Networks

LoRa Long Range

LoRaWAN LoRa Wide Area Network

MAB Multi-Armed Bandit

MAC Medium Access Control

MC Markov Chain

MCL Maximum Coupling Loss

MDP Markov Decision Process

MIOTY MY IoT

ML Machine Learning

MQTT Message Queuing Telemetry Transport

MQTT-SN MQTT for Sensor Networks

MoT MAC on Time

NB-IoT Narrowband-IoT

NIST National Institute of Standards and Technology

NR New Radio

NS Network Server

NoT Network of Things

OASIS Organization for the Advancement of Structured

Information Standards

OCI Oracle Cloud Infrastructure

OFDMA Orthogonal-FDMA

OPC Open Platform Communications

OPC-UA OPC-Unified Architecture

OQPSK Offset Quadrature Phase Shift Keying

OS Operating System

OSI Open Systems Interconnection

OTA Over The Air

OTAA Over The Air Activation

OpEx Operational Expenditure

PDR Packet Delivery Ratio

PER Packet Error Rate

PLR Packet Loss Rate

PRB Physical Resource Block

PRR Packet Reception Rate

PSM Power Saving Mode

PaaS Platform-as-a-Service

QPSK Quadrature Phase Shift Keying

QoS Quality of Service

RAMI4.0 Reference Architectural Model Industrie 4.0

RFDMA Random-FDMA

RNN Random Neural Network

RPL Routing Protocol for Low-Power and Lossy Networks

RPMA Random Phase Multiple Access

RSSI Received Signal Strength Indication

RedCap Reduced Capability

SC-FDMA Single Carrier-FDMA

SDN Software-Defined Networking

SER Symbol Error Rate

SF Spreading Factor

SINR Signal to Interference and Noise Ratio

SIR Signal to Interference Ratio

SNMP Simple Network Management Protocol

SNR Signal to Noise Ratio

SON Self Organizing Network

SS Spread Spectrum

SSL Secure Sockets Layer

SaaS Software-as-a-Service 
TABLE $X$

ACRONYMS TABLE (CONT'D)

\begin{tabular}{ll} 
TDMA & Time Division Multiple Access \\
TLS & Transport Layer Security \\
TM & Transmission Modes \\
TOSSIM & TinyOS Simulator \\
TS & Telegram Splitting \\
TS-UNB & Telegram Splitting Ultra Narrow Band \\
TSMA & Telegram Splitting Multiple Access \\
TTN & The Things Network \\
TVWS & TV White Space \\
ToA & Time On Air \\
UDP & User Datagram Protocol \\
UL & Uplink \\
UNB & Ultra Narrow Band \\
V2X & Vehicle to Everything \\
VPN & Virtual Private Network \\
VoLTE & Voice Over LTE \\
WG & Working Groups \\
WSAN & Wireless Sensor and Actuator Network \\
WSN & Wireless Sensor Network \\
WoT & Web of Things \\
XML & eXtensible Markup Language \\
XaaS & Everything-as-a-Service \\
eDRX & enhanced Discontinuous Reception \\
mMTC & massive Machine Type Communications \\
\hline \hline
\end{tabular}

\section{REFERENCES}

[1] ITU-R, "Framework and overall objectives of the future development of IMT for 2020 and beyond," International Telecommunication Union (ITU), Recommendation M.2083-0, September 2015. [Online]. Available: https://www.itu.int/dms_pubrec/ itu-r/rec/m/R-REC-M.2083-0-201509-I!!PDF-E.pdf

[2] A. Al-Fuqaha, M. Guizani, M. Mohammadi, M. Aledhari, and M. Ayyash, "Internet of Things: A Survey on Enabling Technologies, Protocols, and Applications," IEEE Communications Surveys Tutorials, vol. 17, no. 4, pp. 2347-2376, 2015.

[3] ITU-T, "Overview of the Internet of things," International Telecommunication Union (ITU), Recommendation Y.2060, June 2012, version 1.0. [Online]. Available: https://www.itu.int/rec/T-REC-Y. 2060-201206-I

[4] Ericsson, "Ericsson Mobility Report - June 2021," Ericsson, Tech. Rep., June 2021.

[5] J. DeFranco, "12 Flavors of IoT," Computer, vol. 54, no. 10, pp. 133 137, 2021.

[6] National Institute of Standards and Technology, "Networks of 'Things'," U.S. Department of Commerce, Washington, D.C., Tech. Rep. NIST Special Publication 800-183, July 2016.

[7] Cisco, "Cisco At-a-Glance Internet of Things," Cisco, Tech. Rep., 2016.

[8] 3GPP, "Study on support of reduced capability NR devices (Release 17)," 3rd Generation Partnership Project (3GPP), Technical Report (TR) 38.875, March 2021, version 17.0.0. [Online]. Available: https://portal.3gpp.org/desktopmodules/ Specifications/SpecificationDetails. aspx ?specificationId $=3733$

[9] S. Farrell, "Low-Power Wide Area Network (LPWAN) Overview," Internet Requests for Comments, RFC Editor, RFC 8376, May 2018. [Online]. Available: https://tools.ietf.org/rfc/rfc8376.txt

[10] H. Xu, W. Yu, D. Griffith, and N. Golmie, "A Survey on Industrial Internet of Things: A Cyber-Physical Systems Perspective," IEEE Access, vol. 6, pp. 78 238-78 259, 2018.

[11] C. Bormann, M. Ersue, A. Keranen, "Terminology for ConstrainedNode Networks," Internet Requests for Comments, RFC Editor, RFC 7728, May 2014. [Online]. Available: https://tools.ietf.org/html/rfc7228

[12] A. Augustin, J. Yi, T. Clausen, and W. M. Townsley, "A Study of LoRa: Long Range \& Low Power Networks for the Internet of Things," Sensors, vol. 16, no. 9, 2016. [Online]. Available: https://www.mdpi.com/1424-8220/16/9/1466

[13] K. E. Nolan, W. Guibene, and M. Y. Kelly, "An evaluation of low power wide area network technologies for the Internet of Things," in 2016 International Wireless Communications and Mobile Computing Conference (IWCMC), 2016, pp. 439-444.

[14] M. Centenaro, L. Vangelista, A. Zanella, and M. Zorzi, "Long-range communications in unlicensed bands: The rising stars in the IoT and smart city scenarios," IEEE Wireless Communications, vol. 23, no. 5 , 2016.

[15] A. A. Boulogeorgos, P. D. Diamantoulakis, and G. K. Karagiannidis, "Low Power Wide Area Networks (LPWANs) for Internet of Things (IoT) Applications: Research Challenges and Future Trends," CoRR, vol. abs/1611.07449, 2016. [Online]. Available: http://arxiv.org/abs/ 1611.07449

[16] U. Raza, P. Kulkarni, and M. Sooriyabandara, "Low Power Wide Area Networks: An Overview," IEEE Communications Surveys and Tutorials, vol. 19, no. 2, 2017.

[17] R. Sinha, Y. Wei, and S. Hwang, "A survey on LPWA technology: LoRa and NB-IoT," ICT Express, vol. 3, no. 1, 2017.

[18] H. Wang and A. O. Fapojuwo, "A Survey of Enabling Technologies of Low Power and Long Range Machine-to-Machine Communications," IEEE Communications Surveys Tutorials, vol. 19, no. 4, pp. 26212639, 2017.

[19] K. Mekki, E. Bajic, F. Chaxel, and F. Meyer, "A comparative study of LPWAN technologies for large-scale IoT deployment," ICT Express, vol. 5, no. 1, 2019.

[20] W. Ayoub, A. E. Samhat, F. Nouvel, M. Mroue, and J. C. Prévotet, "Internet of Mobile Things: Overview of LoRaWAN, DASH7, and NBIoT in LPWANs Standards and Supported Mobility," pp. 1561-1581, apr 2019.

[21] J. Finnegan and S. Brown, "A Comparative Survey of LPWA Networking," CoRR, vol. abs/1802.04222, 2018. [Online]. Available: http://arxiv.org/abs/1802.04222

[22] Q. M. Qadir, T. A. Rashid, N. K. Al-Salihi, B. Ismael, A. A. Kist, and Z. Zhang, "Low Power Wide Area Networks: A Survey of Enabling Technologies, Applications and Interoperability Needs," IEEE Access, vol. 6, pp. 77 454-77 473, 2018.

[23] NetWorld2020 ETP, "Smart Networks in the context of NGI," September 2020.

[24] I. F. Akyildiz, Weilian Su, Y. Sankarasubramaniam, and E. Cayirci, "A survey on sensor networks," IEEE Communications Magazine, vol. 40, no. 8, pp. 102-114, 2002

[25] J. Petajajarvi, K. Mikhaylov, A. Roivainen, T. Hanninen, and M. Pettissalo, "On the coverage of LPWANs: range evaluation and channel attenuation model for LoRa technology," in 2015 14th International Conference on ITS Telecommunications (ITST), 2015, pp. 55-59.

[26] 3GPP, "Release 15 Description; Summary of Rel-15 Work Items," 3rd Generation Partnership Project (3GPP), Technical Report (TR) 21.915, September 2019, version 15.0.0. [Online]. Available: https://portal.3gpp.org/desktopmodules/Specifications/ SpecificationDetlails.aspx?specificationId=3389

[27] S. Tabbane, "IoT Standards Part II: 3GPP Standards," Training on Planning Internet of Things (IoTs) Networks, Bandung, September 2018.

[28] P. Reininger, "3GPP Standards for the Internet-of-Things," IoT Business \& Technologies Congress, Singapore, November 2016, Accessed: 04 October 2021. [Online]. Available: https://www.3gpp. org/imlages/presentations/2016_11_3gpp_Standards_for_IoT.pdf

[29] 3GPP, "Release 14 Description; Summary of Rel-14 Work Items," 3rd Generation Partnership Project (3GPP), Technical Report (TR) 21.914, May 2018, version 14.0.0. [Online]. Available: https://portal.3gpp.org/desktopmodules/Specifications/ SpecificationDetlails.aspx? specificationId $=3179$

[30] —, "Release 16 Description; Summary of Rel-16 Work Items," 3rd Generation Partnership Project (3GPP), Technical Report (TR) 21.916, December 2020, version 1.0.0. [Online]. Available: https://portal.3gpp.org/desktopmodules/Specifications/ SpecificationDetlails. aspx?specificationId $=3493$

[31] S. Narayanan, D. Tsolkas, N. Passas, and L. Merakos, "NB-IoT: A Candidate Technology for Massive IoT in the 5G Era," in 2018 IEEE 23rd International Workshop on Computer Aided Modeling and Design of Communication Links and Networks (CAMAD), Sep. 2018, pp. 1-6.

[32] Y. . E. Wang, X. Lin, A. Adhikary, A. Grovlen, Y. Sui, Y. Blankenship, J. Bergman, and H. S. Razaghi, "A Primer on 3GPP Narrowband Internet of Things," IEEE Communications Magazine, vol. 55, no. 3 , pp. 117-123, March 2017.

[33] S. Narayanan, D. Tsolkas, N. Passas, and L. Merakos, "ADAM: An Adaptive Access Mechanism for NB-IoT Systems in the 5G Era," IEEE Access, vol. 9, pp. 109915-109931, 2021.

[34] ETSI, "Short Range Devices; Low Throughput Networks (LTN); Protocols for radio interface A," European Telecommunications Standards Institute (ETSI), Technical Specification (TS) 103 357, June 2018, version 1.1.1. [Online]. Available: https://portal.etsi.org/webapp/ WorkProgram/Report_WorkItem.asp?WKI_ID=45958 
[35] G. Kilian, H. Petkov, R. Psiuk, H. Lieske, F. Beer, J. Robert, and A. Heuberger, "Improved Coverage for Low-Power Telemetry Systems using Telegram Splitting," in Smart SysTech 2013; European Conference on Smart Objects, Systems and Technologies, June 2013, pp. 1-6.

[36] G. Kilian, M. Breiling, H. H. Petkov, H. Lieske, F. Beer, J. Robert, and A. Heuberger, "Increasing Transmission Reliability for Telemetry Systems Using Telegram Splitting," IEEE Transactions on Communications, vol. 63, no. 3, pp. 949-961, March 2015.

[37] T. J. Myers, "Random phase multiple access system with meshing," U.S. Patent US7 773664B2, December 29, 2008, Accessed: 04 October 2021. [Online]. Available: https://patents.google.com/patent/ US7773664B2/en

[38] S. O. Olatinwo and T. Joubert, "Enabling Communication Networks for Water Quality Monitoring Applications: A Survey," IEEE Access, vol. 7, pp. 100332-100362, 2019.

[39] Ingenu, "RPMA Technology for the Internet of Things," 2016

[40] A. Ikpehai, B. Adebisi, K. M. Rabie, K. Anoh, R. E. Ande, M. Hammoudeh, H. Gacanin, and U. M. Mbanaso, "Low-Power Wide Area Network Technologies for Internet-of-Things: A Comparative Review," IEEE Internet of Things Journal, vol. 6, no. 2, pp. 2225-2240, April 2019.

[41] Ingenu, "An Educational Guide How RPMA Works," 2016, Accessed: 05 October 2021. [Online]. Available: https://www.telecomnews.co.il/imalge/users/228328/ftp/my_files/ General\%20Files/IoT\%20Ingenu\%20origlinal.pdf?id=27645739

[42] K. Garvin, "Ingenu Refines Market Strategy to Focus on Enhanced RPMA Technology Development and IoT Platform as a Service Solution," press Release, 14 March 2018, Accessed: 05 October 2021. [Online]. Available: https://www.ingenu.com/2018/03/ ingenu-refines-market-strategy-to-focus-on-enhanced-rpma-technolog y-development-and-iot-platform-as-a-service-solution/

[43] B. Ray, "What Is Weightless?" link Labs, 23 November 2015, Accessed: 05 October 2021. [Online]. Available: https://www. link-labs.com/blog/what-is-weightless

[44] Weightless, "Weightless-P System Specification," November 2017.

[45] E. Kail, A. Banati, E. Lászlo, and M. Kozlovszky, "Security Survey of Dedicated IoT Networks in the Unlicensed ISM Bands," in 2018 IEEE 12th International Symposium on Applied Computational Intelligence and Informatics (SACI), May 2018, pp. $000449-000454$.

[46] S. Tabbane, "Internet of Things: A technical overview of the ecosystem," Regional Workshop for Africa on "Developing the ICT ecosystem to harness Internet-of-Things (IoT)", Mauritius, June 2017, Accessed: 05 October 2021. [Online]. Available: https://www.itu. int/en/ITU-D/Regional-Presence/Africa/Documents/IoT_Technical\% 20Overview\%20of\%20thel\%20Ecosystem_v2_27062017.pdf

[47] Wize Alliance, "Wize LAN PROTOCOL SPECIFICATION V1.2," November 2020

[48] — - "Wize REGIONAL PARAMETERS V1.1," June 2019.

[49] RC1701HP-WIZE Datasheet. Accessed: 05 October 2021. [Online]. Available: https://www.diltronic.com/wp-content/uploads/ 2020/03/DILTROINIC-RC1701HP-WIZE-datasheet.pdf

[50] Sigfox, "Sigfox Technical Overview," May 2017.

[51] "Eutelsat kicks off ELO, its constellation of nanosatellites dedicated to the Internet of Things". Press Release, Accessed: 05 October 2021. [Online]. Available: https://www.eutelsat.com/en/news/press.html\#/pressreleases/ eutelsat-kicks-off-elo-its-constellation-of-nanosatellites-dedicated-to-। the-internet-of-things-2923247

[52] Semtech, "LoRa ${ }^{\mathrm{TM}}$ Modulation Basics," Semtech, Application Note AN1200.22, May 2015, Revision 2.

[53] LoRa Alliance, "LoRaWAN® Regional Parameters," LoRa Alliance, Technical Specification RP002-1.0.3, May 2021. [Online]. Available: https://lora-alliance.org/wp-content/uploads/2021/ 05/RP002-1.0.3-FINAL-1.pdf

[54] Semtech, "SX1272/3/6/7/8: LoRa Modem Designer's Guide," Semtech, Application Note AN1200.13, July 2013, Revision 1.

[55] M. Bor, U. Roedig, T. Voigt, and J. Alonso, "Do LoRa low-power wide-area networks scale?" in MSWiM '16 Proceedings of the 19th ACM International Conference on Modeling, Analysis and Simulation of Wireless and Mobile Systems. ACM Press, Nov. 2016, pp. 5967, a Corrected version. A bug was found in the simulator, whereby previously collided packets would be in some cases marked as "not collided', thereby overestimating the goodput of the network. The bug was fixed and all figures and numbers have been updated accordingly. The overall conclusions, however, has stayed the same.
[56] LoRa Alliance, "LoRaWAN® Specification,” LoRa Alliance, Technical Specification 1.0.4, October 2020. [Online]. Available: https:// lora-alliance.org/resource_hub/lorawan-104-specification-package/

[57] D. Magrin, M. Centenaro, and L. Vangelista, "Performance evaluation of LoRa networks in a smart city scenario," in 2017 IEEE International Conference on Communications (ICC), 2017, pp. 1-7.

[58] T. Polonelli, D. Brunelli, A. Marzocchi, and L. Benini, "Slotted ALOHA on LoRaWAN-design, analysis, and deployment," Sensors (Switzerland), vol. 19, no. 4, feb 2019.

[59] D. Croce, M. Gucciardo, S. Mangione, G. Santaromita, and I. Tinnirello, "Impact of LoRa Imperfect Orthogonality: Analysis of LinkLevel Performance," IEEE Communications Letters, vol. 22, no. 4, 2018.

[60] A. Waret, M. Kaneko, A. Guitton, and N. El Rachkidy, "LoRa Throughput Analysis with Imperfect Spreading Factor Orthogonality," IEEE Wireless Communications Letters, vol. 8, no. 2, pp. 408-411, apr 2019.

[61] R. Piyare, A. Murphy, M. Magno, and L. Benini, "On-Demand LoRa: Asynchronous TDMA for Energy Efficient and Low Latency Communication in IoT," Sensors, vol. 18, p. 3718, 112018.

[62] M. El-Aasser, R. Badawi, M. Ashour, and T. Elshabrawy, "Examining Carrier Sense Multiple Access to Enhance LoRa IoT Network Performance for Smart City Applications," in 2019 IEEE 9th International Conference on Consumer Electronics (ICCE-Berlin), 2019, pp. 168173.

[63] G. Hassan and H. S. Hassanein, "MoT: A Deterministic Latency MAC Protocol for Mission-Critical IoT Applications," in 2018 14th International Wireless Communications and Mobile Computing Conference, IWCMC 2018. IEEE, 2018, pp. 588-593.

[64] D. Zorbas, K. Abdelfadeel, P. Kotzanikolaou, and D. Pesch, "TS-LoRa: Time-slotted LoRaWAN for the Industrial Internet of Things," Computer Communications, vol. 153, pp. 1-10, 2020. [Online]. Available: https://www.sciencedirect.com/science/article/pii/ S0140366419314677

[65] L. Beltramelli, A. Mahmood, P. Österberg, M. Gidlund, P. Ferrari, and E. Sisinni, "Energy Efficiency of Slotted LoRaWAN Communication With Out-of-Band Synchronization," IEEE Transactions on Instrumentation and Measurement, vol. 70, pp. 1-11, 2021.

[66] Nwave website. Accessed: 05 October 2021. [Online]. Available: https://www.nwave.io/

[67] Eltres website. Accessed: 05 October 2021. [Online]. Available: https://www.sony-semicon.co.jp/products_en/eltres/index.html

[68] Amazon, "Amazon Sidewalk Privacy and Security Whitepaper," Amazon, Whitepaper, 2020.

[69] A. Saifullah, M. Rahman, D. Ismail, C. Lu, R. Chandra, and J. Liu, "SNOW: Sensor Network over White Spaces," in Proceedings of the 14th ACM Conference on Embedded Network Sensor Systems CD-ROM, ser. SenSys '16. New York, NY, USA: Association for Computing Machinery, 2016, p. 272-285. [Online]. Available: https://doi.org/10.1145/2994551.2994552

[70] Wi-Fi Alliance, "Wi-Fi HaLow ${ }^{\mathrm{TM}}$ Technology Overview," Wi-Fi Alliance, Technology Overview, 2020.

[71] DASH7 Alliance, "DASH7 Alliance Wireless Sensor and Actuator Network Protocol," 2018, version 1.2.

[72] IoT Analytics website. Accessed: 05 October 2021. [Online]. Available: https://iot-analytics.com/

[73] Semtech, "LoRa® and LoRaWAN®: A Technical Overview," Semtech, Technical Paper, December 2019.

[74] LoRa Alliance, "FUOTA Process Summary Technical Recommendation," LoRa Alliance, Technical Recommendation TR002, January 2019, v1.0.0. [Online]. Available: https://lora-alliance.org/sites/default/ files/2019-04/tr002-fuota_process_summary-v1.0.0.pdf

[75] Semtech, "Understanding the LoRa ${ }^{\circledR}$ Adaptive Data Rate," Semtech, Technical Paper, December 2019.

[76] — "LoRaWAN® Mobile Applications: Blind ADR," Semtech, Technical Paper, December 2019.

[77] S. Li, U. Raza, and A. Khan, "How Agile is the Adaptive Data Rate Mechanism of LoRaWAN?" in 2018 IEEE Global Communications Conference (GLOBECOM), 2018, pp. 206-212.

[78] K. Kousias, G. Caso, O. Alay, and F. Lemic, "Empirical Analysis of LoRaWAN Adaptive Data Rate for Mobile Internet of Things Applications," in Proceedings of the 2019 on Wireless of the Students, by the Students, and for the Students Workshop, ser. S3'19. New York, NY, USA: Association for Computing Machinery, 2019, p. 9-11. [Online]. Available: https://doi.org/10.1145/3349621.3355727 
[79] R. Kufakunesu, G. Hancke, and A. Abu-Mahfouz, "A Survey on Adaptive Data Rate Optimization in LoRaWAN: Recent Solutions and Major Challenges," Sensors (Basel, Switzerland), vol. 20, September 2020.

[80] T. Bouguera, J.-F. Diouris, J.-J. Chaillout, R. Jaouadi, and G. Andrieux, "Energy Consumption Model for Sensor Nodes Based on LoRa and LoRaWAN," Sensors (Basel, Switzerland), vol. 18, June 2018.

[81] LoRa Alliance, "LoRaWAN® Backend Interfaces Technical Specification," LoRa Alliance, Technical Specification TS002-1.1.0, October 2020. [Online]. Available: https://lora-alliance.org/wp-content/ uploads/2020/11/TS002-1.1.0_LoRaWAN_Backend_Interfaces.pdf

[82] G. Boquet, P. Tuset-Peiró, F. Adelantado, T. Watteyne, and X. Vilajosana, "LR-FHSS: Overview and Performance Analysis," IEEE Communications Magazine, vol. 59, no. 3, pp. 30-36, 2021.

[83] LoRa Alliance vertical markets. Accessed: 05 October 2021. [Online]. Available: https://lora-alliance.org/lorawan-vertical-markets/

[84] LoRa Alliance website. Accessed: 05 October 2021. [Online]. Available: https://lora-alliance.org/

[85] I. Butun, N. Pereira, and M. Gidlund, "Security risk analysis of LoRaWAN and future directions," Future Internet, vol. 11, no. 1, pp. $1-22,2018$.

[86] M. Krichen, M. Lahami, O. Cheikhrouhou, R. Alroobaea, and A. Jmal Maâlej, Security Testing of Internet of Things for Smart City Applications: A Formal Approach, January 2020, pp. 629-653.

[87] M. Stoyanova, Y. Nikoloudakis, S. Panagiotakis, E. Pallis, and E. K. Markakis, "A Survey on the Internet of Things (IoT) Forensics: Challenges, Approaches, and Open Issues," IEEE Communications Surveys Tutorials, vol. 22, no. 2, pp. 1191-1221, 2020

[88] O. B. A. Seller and N. Sornin, "Low power long range transmitter," Europe Patent EP2 763321A1, February 05, 2013, Accessed: 05 October 2021. [Online]. Available: https://patents.google.com/patent/ EP2763321A1/en

[89] O. B. A. Seller, "Wireless communication method," Europe Patent EP3 002 884B1, September 30, 2014, Accessed: 05 October 2021. [Online]. Available: https://patents.google.com/patent/EP3002884B1/ en

[90] "A Brief History of LoRa®: Three Inventors Share Their Personal Story at The Things Conference". Accessed: 05 October 2021. [Online]. Available: https://blog.semtech.com/a-brief-history-of-lora-। three-inventors-share-their-personal-story-at-the-things-conference

[91] J. P. Shanmuga Sundaram, W. Du, and Z. Zhao, "A Survey on LoRa Networking: Research Problems, Current Solutions, and Open Issues," IEEE Communications Surveys Tutorials, vol. 22, no. 1, pp. 371-388, 2020.

[92] F. Adelantado, X. Vilajosana, P. Tuset-Peiro, B. Martinez, J. MeliaSegui, and T. Watteyne, "Understanding the Limits of LoRaWAN," IEEE Communications Magazine, vol. 55, no. 9, pp. 34-40, 2017

[93] S. M. Danish, M. Lestas, W. Asif, H. K. Qureshi, and M. Rajarajan, "A Lightweight Blockchain Based Two Factor Authentication Mechanism for LoRaWAN Join Procedure," in 2019 IEEE International Conference on Communications Workshops (ICC Workshops), 2019, pp. 1-6.

[94] N. Matni, J. Moraes, H. Oliveira, D. Rosário, and E. Cerqueira, "LoRaWAN Gateway Placement Model for Dynamic Internet of Things Scenarios," Sensors, vol. 20, no. 15, 2020. [Online]. Available: https://www.mdpi.com/1424-8220/20/15/4336

[95] S. Messaoud, A. Bradai, O. B. Ahmed, P. T. A. Quang, M. Atri, and M. S. Hossain, "Deep Federated Q-Learning-Based Network Slicing for Industrial IoT," IEEE Transactions on Industrial Informatics, vol. 17, no. 8, pp. 5572-5582, 2021

[96] S. Dawaliby, A. Bradai, and Y. Pousset, "Distributed Network Slicing in Large Scale IoT Based on Coalitional Multi-Game Theory," IEEE Transactions on Network and Service Management, vol. 16, no. 4, pp. 1567-1580, 2019.

[97] K. Kritsis, G. Z. Papadopoulos, A. Gallais, P. Chatzimisios, and F. Théoleyre, "A Tutorial on Performance Evaluation and Validation Methodology for Low-Power and Lossy Networks," IEEE Communications Surveys Tutorials, vol. 20, no. 3, pp. 1799-1825, 2018.

[98] M. Saari, A. M. bin Baharudin, P. Sillberg, S. Hyrynsalmi, and W. Yan, "LoRa - A survey of recent research trends," in 2018 41st International Convention on Information and Communication Technology, Electronics and Microelectronics (MIPRO), 2018, pp. 0872-0877.

[99] K. Christidis and M. Devetsikiotis, "Blockchains and Smart Contracts for the Internet of Things," IEEE Access, vol. 4, pp. 2292-2303, 2016.

[100] M. S. Ali, M. Vecchio, M. Pincheira, K. Dolui, F. Antonelli, and M. H. Rehmani, "Applications of Blockchains in the Internet of Things: A Comprehensive Survey," IEEE Communications Surveys Tutorials, vol. 21, no. 2, pp. 1676-1717, 2019.
[101] S. K. Goudos, P. I. Dallas, S. Chatziefthymiou, and S. Kyriazakos, "A Survey of IoT Key Enabling and Future Technologies: 5G, Mobile IoT, Sematic Web and Applications," Wireless Personal Communications, vol. 97, no. 2, pp. 1645-1675, Nov 2017. [Online]. Available: https://doi.org/10.1007/s11277-017-4647-8

[102] M. Jacoby and T. Usländer, "Digital Twin and Internet of Things-Current Standards Landscape," Applied Sciences, vol. 10, no. 18, 2020. [Online]. Available: https://www.mdpi.com/2076-3417/ $10 / 18 / 6519$

[103] A. Rasheed, O. San, and T. Kvamsdal, "Digital Twin: Values, Challenges and Enablers From a Modeling Perspective," IEEE Access, vol. 8, pp. 21 980-22012, 2020.

[104] A. Fuller, Z. Fan, C. Day, and C. Barlow, "Digital Twin: Enabling Technologies, Challenges and Open Research," IEEE Access, vol. 8 , pp. 108952-108971, 2020.

[105] Y. Wu, K. Zhang, and Y. Zhang, "Digital Twin Networks: A Survey," IEEE Internet of Things Journal, vol. 8, no. 18, pp. 13789-13804, 2021.

[106] T. Qiu, J. Chi, X. Zhou, Z. Ning, M. Atiquzzaman, and D. O. Wu, "Edge Computing in Industrial Internet of Things: Architecture, Advances and Challenges," IEEE Communications Surveys Tutorials, vol. 22, no. 4, pp. 2462-2488, 2020.

[107] K. Tange, M. De Donno, X. Fafoutis, and N. Dragoni, “A Systematic Survey of Industrial Internet of Things Security: Requirements and Fog Computing Opportunities," IEEE Communications Surveys Tutorials, vol. 22, no. 4, pp. 2489-2520, 2020.

[108] J. Haxhibeqiri, E. De Poorter, I. Moerman, and J. Hoebeke, "A Survey of LoRaWAN for IoT: From Technology to Application," Sensors, vol. 18, no. 11, 2018. [Online]. Available: https://www.mdpi.com/ $1424-8220 / 18 / 11 / 3995$

[109] M. A. Ertürk, M. A. Aydın, M. T. Büyükakkaşlar, and H. Evirgen, "A Survey on LoRaWAN Architecture, Protocol and Technologies," Future Internet, vol. 11, no. 10, 2019. [Online]. Available: https://www.mdpi.com/1999-5903/11/10/216

[110] J. M. Marais, A. M. Abu-Mahfouz, and G. P. Hancke, "A Review of LoRaWAN Simulators: Design Requirements and Limitations," in 2019 International Multidisciplinary Information Technology and Engineering Conference (IMITEC), 2019, pp. 1-6.

[111] O. Afisiadis, S. Li, J. Tapparel, A. Burg, and A. Balatsoukas-Stimming, "On the Advantage of Coherent LoRa Detection in the Presence of Interference," IEEE Internet of Things Journal, pp. 1-1, 2021.

[112] B. Reynders and S. Pollin, "Chirp spread spectrum as a modulation technique for long range communication," in 2016 Symposium on Communications and Vehicular Technologies (SCVT), 2016, pp. 1-5.

[113] L. Vangelista, "Frequency Shift Chirp Modulation: The LoRa Modulation," IEEE Signal Processing Letters, vol. 24, no. 12, pp. 1818-1821, 2017.

[114] E. M. Rochester, A. M. Yousuf, B. Ousat, and M. Ghaderi, "Lightweight Carrier Sensing in LoRa: Implementation and Performance Evaluation," in ICC 2020 - 2020 IEEE International Conference on Communications (ICC), 2020, pp. 1-6.

[115] O. Georgiou and U. Raza, "Low Power Wide Area Network Analysis: Can LoRa Scale?" IEEE Wireless Communications Letters, vol. 6 , no. 2, pp. 162-165, 2017

[116] J. Toussaint, N. El Rachkidy, and A. Guitton, "Performance analysis of the on-the-air activation in LoRaWAN," in 2016 IEEE 7th Annual Information Technology, Electronics and Mobile Communication Conference (IEMCON), 2016, pp. 1-7.

[117] A. Biason and M. Zorzi, "On the effects of battery imperfections in an energy harvesting device," in 2016 International Conference on Computing, Networking and Communications (ICNC), 2016, pp. 1-7.

[118] C. Delgado, J. M. Sanz, C. Blondia, and J. Famaey, "Batteryless LoRaWAN Communications Using Energy Harvesting: Modeling and Characterization," IEEE Internet of Things Journal, vol. 8, no. 4, pp. 2694-2711, 2021.

[119] L. Casals, B. Mir, R. Vidal, and C. Gomez, "Modeling the Energy Performance of LoRaWAN," Sensors, vol. 17, no. 10, 2017. [Online]. Available: https://www.mdpi.com/1424-8220/17/10/2364

[120] R. S. Sutton and A. G. Barto, Reinforcement Learning: An Introduction, 2nd ed. The MIT Press, 1998

[121] T. Voigt, M. Bor, U. Roedig, and J. Alonso, "Mitigating Inter-Network Interference in LoRa Networks," in EWSN '17 Proceedings of the 2017 International Conference on Embedded Wireless Systems and Networks. ACM Press, Feb. 2017, pp. 323-328, (C) Junction Publishing, 2017. This is the author's version of the work. It is posted here for your personal use. Not for redistribution. The definitive Version of Record 
was published in EWSN '17 Proceedings of the 2017 International Conference on Embedded Wireless Systems and Networks.

[122] A. Pop, U. Raza, P. Kulkarni, and M. Sooriyabandara, "Does Bidirectional Traffic Do More Harm Than Good in LoRaWAN Based LPWA Networks?" GLOBECOM 2017 - 2017 IEEE Global Communications Conference, pp. 1-6, 2017.

[123] G. Callebaut, G. Ottoy, and L. van der Perre, "Cross-Layer Framework and Optimization for Efficient Use of the Energy Budget of IoT Nodes," in 2019 IEEE Wireless Communications and Networking Conference (WCNC), 2019, pp. 1-6.

[124] LoRaMACSim. Accessed: 05 October 2021. [Online]. Available: https://github.com/Beltra90/LoRaMACSim

[125] D.-T. Ta, K. Khawam, S. Lahoud, C. Adjih, and S. Martin, "LoRaMAB: A Flexible Simulator for Decentralized Learning Resource Allocation in IoT Networks," in 2019 12th IFIP Wireless and Mobile Networking Conference (WMNC), 2019, pp. 55-62.

[126] K. Q. Abdelfadeel, D. Zorbas, V. Cionca, and D. Pesch, “ FREE -Fine-Grained Scheduling for Reliable and Energy-Efficient Data Collection in LoRaWAN," IEEE Internet of Things Journal, vol. 7, no. 1 , pp. 669-683, 2020.

[127] R. Marini, K. Mikhaylov, G. Pasolini, and C. Buratti, "LoRaWANSim A Flexible Simulator for LoRaWAN Networks," Sensors, vol. 21, no. 3, 2021. [Online]. Available: https://www.mdpi.com/1424-8220/21/3/695

[128] A. M. Yousuf, E. M. Rochester, B. Ousat, and M. Ghaderi, "Throughput, Coverage and Scalability of LoRa LPWAN for Internet of Things," in 2018 IEEE/ACM 26th International Symposium on Quality of Service (IWQoS), 2018, pp. 1-10.

[129] M. Slabicki, G. Premsankar, and M. Di Francesco, "Adaptive configuration of lora networks for dense IoT deployments," in NOMS 2018 - 2018 IEEE/IFIP Network Operations and Management Symposium, 2018, pp. 1-9.

[130] M. Capuzzo, D. Magrin, and A. Zanella, "Confirmed traffic in LoRaWAN: Pitfalls and countermeasures," in 2018 17th Annual Mediterranean Ad Hoc Networking Workshop (Med-Hoc-Net), 2018, pp. 1-7.

[131] D. Magrin, M. Capuzzo, and A. Zanella, "A Thorough Study of LoRaWAN Performance Under Different Parameter Settings," IEEE Internet of Things Journal, vol. 7, no. 1, pp. 116-127, 2020.

[132] J. Finnegan, S. Brown, and R. Farrell, "Modeling the Energy Consumption of LoRaWAN in ns-3 Based on Real World Measurements," in 2018 Global Information Infrastructure and Networking Symposium (GIIS), 2018, pp. 1-4.

[133] F. Van den Abeele, J. Haxhibeqiri, I. Moerman, and J. Hoebeke, "Scalability Analysis of Large-Scale LoRaWAN Networks in ns-3," IEEE Internet of Things Journal, vol. 4, no. 6, pp. 2186-2198, 2017.

[134] T.-H. To and A. Duda, "Simulation of LoRa in NS-3: Improving LoRa Performance with CSMA," in 2018 IEEE International Conference on Communications (ICC), 2018, pp. 1-7.

[135] S. Fahmida, V. P. Modekurthy, M. Rahman, A. Saifullah, and M. Brocanelli, "Long-Lived LoRa: Prolonging the Lifetime of a LoRa Network," in 2020 IEEE 28th International Conference on Network Protocols (ICNP), 2020, pp. 1-12.

[136] W. Xu, J. Y. Kim, W. Huang, S. S. Kanhere, S. K. Jha, and W. Hu, "Measurement, Characterization, and Modeling of LoRa Technology in Multifloor Buildings," IEEE Internet of Things Journal, vol. 7, no. 1, pp. 298-310, 2020.

[137] T. Petrić, M. Goessens, L. Nuaymi, L. Toutain, and A. Pelov, "Measurements, performance and analysis of LoRa FABIAN, a real-world implementation of LPWAN," in 2016 IEEE 27th Annual International Symposium on Personal, Indoor, and Mobile Radio Communications (PIMRC), 2016, pp. 1-7.

[138] A. Javed, H. Larijani, and A. Wixted, "Improving Energy Consumption of a Commercial Building with IoT and Machine Learning," IT Professional, vol. 20, no. 5, pp. 30-38, 2018.

[139] L. Hou, K. Zheng, Z. Liu, X. Xu, and T. Wu, "Design and Prototype Implementation of a Blockchain-Enabled LoRa System With Edge Computing," IEEE Internet of Things Journal, vol. 8, no. 4, pp. 2419 2430, 2021.

[140] K. R. Ozyilmaz and A. Yurdakul, "Designing a Blockchain-Based IoT With Ethereum, Swarm, and LoRa: The Software Solution to Create High Availability With Minimal Security Risks," IEEE Consumer Electronics Magazine, vol. 8, no. 2, pp. 28-34, 2019.

[141] S. R. Niya, E. Schiller, I. Cepilov, and B. Stiller, "BIIT: Standardization of Blockchain-based I2oT Systems in the I4 Era," in NOMS 2020 2020 IEEE/IFIP Network Operations and Management Symposium, 2020 , pp. $1-9$

[142] S. Tabbane, "IoT Network Planning," Training on Developing the ICT ecosystem to harness IoTs, Bangkok, December 2016.
[143] F. Österlind, "A Sensor Network Simulator for the Contiki OS," Master's thesis, Swedish Institute of Computer Science, Kista, Sweden, 2016, Accessed: 05 October 2021. [Online]. Available: https://core.ac.uk/download/pdf/300993645.pdf

[144] F. Osterlind, A. Dunkels, J. Eriksson, N. Finne, and T. Voigt, "CrossLevel Sensor Network Simulation with COOJA," in Proceedings. 2006 31st IEEE Conference on Local Computer Networks, 2006, pp. 641648.

[145] A. Dunkels, B. Gronvall, and T. Voigt, "Contiki - a lightweight and flexible operating system for tiny networked sensors," in 29th Annual IEEE International Conference on Local Computer Networks, 2004, pp. $455-462$.

[146] An Introduction to Cooja. Accessed: 05 October 2021. [Online]. Available: https://github.com/contiki-os/contiki/wiki/ An-Introduction-to-Cooja

[147] The Contiki Operating System. Accessed: 05 october 2021. [Online] Available: https://github.com/contiki-os/contiki

[148] Contiki-NG: The OS for Next Generation IoT Devices. Accessed: 05 October 2021. [Online]. Available: https://github.com/contiki-ng/ contiki-ng

[149] L. Sitanayah, C. J. Sreenan, and S. Fedor, "A Cooja-Based Tool for Coverage and Lifetime Evaluation in an In-Building Sensor Network," Journal of Sensor and Actuator Networks, vol. 5, no. 1, 2016. [Online]. Available: https://www.mdpi.com/2224-2708/5/1/4

[150] Mobility of Nodes in Cooja. Accessed: 05 October 2021. [Online]. Available: https://anrg.usc.edu/contiki/index.php/Mobility_of_Nodes_ in_Cooja

[151] P. Levis, N. Lee, M. Welsh, and D. Culler, "TOSSIM: Accurate and Scalable Simulation of Entire TinyOS Applications," in Proceedings of the 1st International Conference on Embedded Networked Sensor Systems, ser. SenSys '03. New York, NY, USA: Association for Computing Machinery, 2003, p. 126-137. [Online]. Available: https://doi.org/10.1145/958491.958506

[152] P. Levis, S. Madden, J. Polastre, R. Szewczyk, K. Whitehouse, A. Woo, D. Gay, J. Hill, M. Welsh, E. Brewer, and D. Culler, TinyOS An Operating System for Sensor Networks. Berlin, Heidelberg: Springer Berlin Heidelberg, 2005, pp. 115-148. [Online]. Available: https://doi.org/10.1007/3-540-27139-2_7

[153] TOSSIM github repository. Accessed: 05 October 2021 [Online]. Available: https://github.com/tinyos/tinyos-main/tree/master/ tos/lib/tossim

[154] TinyOS github repository. Accessed: 05 October 2021. [Online] Available: https://github.com/tinyos/tinyos-main

[155] D. Gay, P. Levis, R. von Behren, M. Welsh, E. Brewer, and D. Culler "The NesC Language: A Holistic Approach to Networked Embedded Systems," SIGPLAN Not., vol. 49, no. 4S, p. 41-51, Jul. 2014. [Online]. Available: https://doi.org/10.1145/2641638.2641652

[156] nesC github repository. Accessed: 05 October 2021. [Online]. Available: https://github.com/tinyos/nesc

[157] V. Shnayder, M. Hempstead, B.-r. Chen, G. W. Allen, and M. Welsh, "Simulating the Power Consumption of Large-Scale Sensor Network Applications," in Proceedings of the 2nd International Conference on Embedded Networked Sensor Systems, ser. SenSys '04. New York, NY, USA: Association for Computing Machinery, 2004, p. 188-200 [Online]. Available: https://doi.org/10.1145/1031495.1031518

[158] A. Derhab, F. Ounini, and B. Remli, "MOB-TOSSIM: An Extension Framework for TOSSIM Simulator to Support Mobility in Wireless Sensor and Actuator Networks," in 2012 IEEE 8th International Conference on Distributed Computing in Sensor Systems, 2012, pp. 300-305.

[159] K. Mehdi, M. Lounis, A. Bounceur, and T. Kechadi, "CupCarbon: A Multi-Agent and Discrete Event Wireless Sensor Network Design and Simulation Tool," in Proceedings of the 7th International ICST Conference on Simulation Tools and Techniques, ser. SIMUTools '14. Brussels, BEL: ICST (Institute for Computer Sciences, SocialInformatics and Telecommunications Engineering), 2014, p. 126-131. [Online]. Available: https://doi.org/10.4108/icst.simutools.2014.254811

[160] A. Bounceur, O. Marc, M. Lounis, J. Soler, L. Clavier, P. Combeau, R. Vauzelle, L. Lagadec, R. Euler, M. Bezoui, and P. Manzoni, "CupCarbon-Lab: An IoT emulator," in 2018 15th IEEE Annual Consumer Communications Networking Conference (CCNC), 2018, pp. $1-2$.

[161] A. Bounceur, L. Clavier, P. Combeau, O. Marc, R. Vauzelle, A. Masserann, J. Soler, R. Euler, T. Alwajeeh, V. Devendra, U. Noreen, E. Soret, and M. Lounis, "CupCarbon: A new platform for the design, simulation and $2 \mathrm{D} / 3 \mathrm{D}$ visualization of radio propagation and interferences in 
IoT networks," in 2018 15th IEEE Annual Consumer Communications Networking Conference (CCNC), 2018, pp. 1-4.

[162] CupCarbon github repository. Accessed: 05 October 2021. [Online]. Available: https://github.com/bounceur/CupCarbon

[163] CupCarbon ${ }^{\circledR}$ User Guide Version U-One 5.1. Accessed: 05 October 2021. [Online]. Available: http://freenwork.com/cupcarbon/cupcarbon_ user_guide.pdf

[164] LoRaWANSim github repository. Accessed: 05 October 2021. [Online] Available: https://github.com/kvmikhayl/LoRaWAN_simulator

[165] LoRaSim. Accessed: 05 October 2021. [Online]. Available: https: //www.lancaster.ac.uk/scc/sites/lora/lorasim.html

[166] LoRaEnergySim github repository. Accessed: 05 October 2021. [Online]. Available: https://github.com/GillesC/LoRaEnergySim

[167] S. L. Duc-Tuyen Ta, Kinda Khawam. LoRaWAN Network Simulator with Reinforcement Learning-based Algorithms. Accessed: 05 October 2021. [Online]. Available: https://github.com/tuyenta/IoT-MAB

[168] LoRaFREE github repository. Accessed: 05 October 2021. [Online] Available: https://github.com/kqorany/FREE

[169] Castalia github repository. Accessed: 05 October 2021. [Online]. Available: https://github.com/boulis/Castalia

[170] PAWiS. Accessed: 05 October 2021. [Online]. Available: http: //pawis.sourceforge.net/

[171] FLoRa. Accessed: 05 October 2021. [Online]. Available: https: //flora.aalto.fi/

[172] FLoRa github repository. Accessed: 05 October 2021. [Online]. Available: https://github.com/mariuszslabicki/flora

[173] ns-3. Accessed: 05 October 2021. [Online]. Available: https: //www.nsnam.org/

[174] ns-3 Documentation. Accessed: 05 October 2021. [Online]. Available: https://www.nsnam.org/documentation

[175] D. Magrin, "Network level performances of a LoRa system," Master's thesis, Università di Padova, Padova, Italy, 2016, Accessed: 05 October 2021. [Online]. Available: http://tesi.cab.unipd.it/53740/

[176] ns-3 gitlab repository. Accessed: 05 October 2021. [Online]. Available: https://gitlab.com/nsnam/ns-3-dev

[177] National Institute of Standards and Technology, "The NIST Definition of Cloud Computing," U.S. Department of Commerce, Washington, D.C., Tech. Rep. NIST Special Publication 800-145, September 2011.

[178] D. C. Marinescu, Cloud Computing: Theory And Practice, 2nd ed. Cambridge, MA, USA: Morgan Kaufmann, 2017.

[179] R. L. Maistre. (2021, April) "AWS and Azure still dominate cloud, but rivals can still thrive and help support DSPs". Accessed: 05 October 2021. [Online]. Available: https://www. telecomtv.com/content/digital-platforms-services/aws-and-azure-still-। dominate-cloud-but-rivals-can-still-thrive-and-help-support-dsps-413। 91/

[180] J. Jiang, V. Sekar, I. Stoica, and H. Zhang, "Unleashing the Potential of Data-Driven Networking," 09 2017, pp. 110-126.

[181] M. Gohar, S. H. Ahmed, M. Khan, N. Guizani, A. Ahmed, and A. Ur Rahman, "A Big Data Analytics Architecture for the Internet of Small Things," IEEE Communications Magazine, vol. 56, no. 2, pp. 128-133, 2018.

[182] M. Meli, E. Gatt, O. Casha, I. Grech, and J. Micallef, "A Low Cost LoRa-based IoT Big Data Capture and Analysis System for Indoor Air Quality Monitoring," in 2020 International Conference on Computational Science and Computational Intelligence (CSCI), 2020, pp. $376-381$

[183] "Alignment Report for Reference Architectural Model for Industrie 4.0/ Intelligent Manufacturing System Architecture," April 2018 Accessed: 04 October 2021. [Online]. Available: https://www.dke. de/resource/blob/1711304/2e4d62811e90ee7aad10eeb6fdeb33d2/align। ment-report-for-reference-architectural-model-for-industrie-4-0-data. pdf

[184] "Reference Architectural Model Industrie 4.0 (RAMI4.0) - An Introduction," August 2018, Accessed: 04 October 2021. [Online]. Available: https://www.plattform-i40.de/IP/Redaktion/EN/Downloads/ Publikation/rami40-an-introduction.pdf? blob=publicationFile\&v=7

[185] "The Industrial Internet of Things Volume G1: Reference Architecture," June 2019, Accessed: 04 October 2021. [Online]. Available: https://www.iiconsortium.org/pdf/IIRA-v1.9.pdf

[186] "International Data Spaces Reference Architecture Model,' April 2019, Accessed: 04 October 2021. [Online]. Available: https://internationaldataspaces.org/wp-content/ uploads/IDS-RAM-3.0-2019.pdf

[187] D. Hanes, G. Salgueiro, P. Grossetete, R. Barton, and J. Henry, IoT Fundamentals: Networking Technologies, Protocols, and Use Cases for the Internet of Things. Cisco Press, 2017.
[188] W3C. Web of Things (WoT) Architecture. Accessed: 05 October 2021. [Online]. Available: https://www.w3.org/TR/wot-architecture/ Overview.html

[189] —. Efficient XML Interchange (EXI) Format 1.0 (Second Edition). Accessed: 05 October 2021. [Online]. Available: https: //www.w3.org/TR/exi/

[190] P. Warden and D. Situnayake, TinyML: Machine Learning with TensorFlow Lite on Arduino and Ultra-Low-Power Microcontrollers, 1st ed. O'Reilly Media, 2020

[191] J. Arm, T. Benesl, P. Marcon, Z. Bradac, T. Schröder, A. Belyaev, T. Werner, V. Braun, P. Kamensky, F. Zezulka, C. Diedrich, and P. Dohnal, "Automated Design and Integration of Asset Administration Shells in Components of Industry 4.0," Sensors, vol. 21, no. 6, 2021. [Online]. Available: https://www.mdpi.com/1424-8220/21/6/2004

[192] IEC 63278-1 ED1 Publications. Accessed: 04 October 2021. [Online]. Available: https://www.iec.ch/ords/f? $\mathrm{p}=103: 38: 503467513783566:::$ : FSP_ORG_ID,FSP_APEX_PAGE,FSP_PROJECT_ID:1250,23, 103536

[193] AASX github repository. Accessed: 04 October 2021. [Online]. Available: https://github.com/admin-shell-io

[194] Eclipse BaSyx website. Accessed: 04 October 2021. [Online]. Available: https://projects.eclipse.org/projects/technology.basyx

[195] open62541 website. Accessed: 04 October 2021. [Online]. Available: https://open62541.org/

[196] Understand twin models in Azure Digital Twins. Accessed: 04 October 2021. [Online]. Available: https://docs.microsoft.com/en-us/ azure/digital-twins/concepts-models

[197] A. Mäkinen, J. Jiménez, and R. Morabito, "ELIoT: Design of an emulated IoT platform," in 2017 IEEE 28th Annual International Symposium on Personal, Indoor, and Mobile Radio Communications (PIMRC), 2017, pp. 1-7.

[198] Emulated IoT (ELIOT) Platform. Accessed: 05 October 2021 [Online]. Available: https://github.com/Alliasd/ELIOT

[199] What is Azure Digital Twins? Accessed: 04 October 2021. [Online]. Available: https://docs.microsoft.com/en-us/azure/ digital-twins/overview

[200] R. Pickholtz, D. Schilling, and L. Milstein, "Theory of SpreadSpectrum Communications - A Tutorial," IEEE Transactions on Communications, vol. 30, no. 5, pp. 855-884, 1982.

[201] J. G. Proakis and M. Salehi, Digital Communications, 5th ed. McGraw-Hill Education, 2007.

Christos Milarokostas received the 4-year B.S. degree and the M.S. degree from the Department of Informatics and Telecommunications, National and Kapodistrian University of Athens (NKUA), in 2018 and 2020, respectively. $\mathrm{He}$ is currently pursuing a $\mathrm{Ph} . \mathrm{D}$. degree in the area of access mechanisms for wireless networks at the aforementioned department. He has participated in a LoRa-focused domestic project and he is now participating in an EC-funded project. He works as a Research Associate at NKUA and his current research interests include design and analysis of networking protocols, network architectures, wireless sensor and actuator networks and information management.

Dimitris Tsolkas received the Ph.D. degree from the Department of Informatics and Telecommunications, National and Kapodistrian University of Athens (NKUA). He has long experience in research and development and project management, working for a plethora of EC-funded research and innovation actions. He also has teaching experience as a Lecturer and an Assistant Instructor with the Department of Informatics and Telecommunications, NKUA, and the Department of Computer Science and Engineering, University of Ioannina. He is currently a Senior Research Fellow at NKUA. So far, he has published more than 40 articles in peer-reviewed journals, international conferences, and book chapters, while he has served as a reviewer for high-quality journals and magazines. With key expertise in $5 \mathrm{G}$ network functions optimization and endto-end network emulation and testing, his current research interests include wireless/mobile communication systems, radio access networks design and analysis, and user experience management in service provisioning. 
Nikos Passas received the Diploma degree (Hons.) from the Department of Computer Engineering, University of Patras, Greece, in 1992, and the Ph.D. degree from the Department of Informatics and Telecommunications, University of Athens, Greece, in 1997. He is currently a member of the Teaching Staff with the Department of Informatics and Telecommunications, University of Athens, where he as a Group Leader of the Green, Adaptive and Intelligent Networking (GAIN) Research Group inside the department. Over the years, he has participated and coordinated a large number of national and European research projects. He has published more than 120 papers in peerreviewed journals and international conferences and has also published one book and 11 book chapters. He has served as a Guest Editor and Technical Program Committee Member for prestigious magazines and conferences, such as IEEE Wireless Communications Magazine, Wireless Communications and Mobile Computing journal, IEEE Vehicular Technology Conference, IEEE PIMRC, and IEEE GLOBECOM.

Lazaros Merakos received the Diploma degree in electrical and mechanical engineering from the National Technical University of Athens, Greece, in 1978, and the M.S. and Ph.D. degrees in electrical engineering from the State University of New York, Buffalo, in 1981 and 1984, respectively. He has been on the Faculty of the University of Connecticut (1983-1986), and Northeastern University, Boston (1986-1994). Since 1994, he has been a Professor with the Department of Informatics and Telecommunications, National and Kapodistrian University of Athens (NKUA), where he has been the Scientific Director of the Network Operations and Management Center. Since June 2020, he has been serving as the Chair for the newly founded Department of Digital Industry Technologies, NKUA. He has been the Principal Investigator for NKUA in many EU funded research and development projects in the area of mobile/wireless networks. His research interests include network technologies, services, and applications, where he has authored more than 300 publications in international journals and conferences. He was a recipient of the Guanella Award for the Best Paper presented at the 1994 International Zurich Conference on Mobile Communications and the Best Paper Award in the 2008 IEEE International Symposium on Communications, Control and Signal Processing. He is the Chairman of the Board of the Greek Universities Network, a non-profit organization for the design and development of advanced ICT services for the 25 universities in Greece. 